\title{
Effect of target thickness in blunt projectile penetration of Weldox 460 E steel plates
}

\author{
Tore Børvik ${ }^{\mathrm{a}, \mathrm{b}, *}$, Odd Sture Hopperstad ${ }^{\mathrm{a}}$, Magnus Langseth ${ }^{\mathrm{a}}$, Kjell Arne Malo \\ ${ }^{a}$ Structural Impact Laboratory (SIMLab), Department of Structural Engineering, Norwegian University of Science and \\ Technology, N-7491 Trondheim, Norway \\ ${ }^{\mathrm{b}}$ Norwegian Defence Estate Agency, Research and Development Section, N-0015 Oslo, Norway
}

Received 1 December 2000; received in revised form 7 August 2002

\begin{abstract}
This paper describes an experimental, analytical and numerical investigation of the penetration and perforation of circular Weldox $460 \mathrm{E}$ steel plates with different thicknesses struck by a blunt projectile at various impact velocities. In the experimental tests, a compressed gas gun was used to launch the sabot mounted projectile at impact velocities well above and just below the ballistic limit of the target plates. Nominal hardness, diameter, length and mass of the projectile were kept constant in all tests. The target plate was clamped in a rigid circular frame, and the thickness was varied between 6 and $30 \mathrm{~mm}$. Measurements were made of the initial and residual velocities, and the ballistic limit velocity and the residual versus impact velocity curve were obtained for each target thickness tested. In addition, a digital high-speed camera system was used to photograph the penetration event. The experimental findings from the tests are presented and discussed, and the results are used to assess some empirical, analytical and numerical models. It is shown that especially the results obtained by the finite element approach are encouraging in terms of predicting the response of the plates examined.
\end{abstract}

(C) 2002 Elsevier Science Ltd. All rights reserved.

Keywords: Impact; Target thickness; Experiments; Empirical models; Analytical models; Numerical simulations

\section{Introduction}

Structural impact problems have become increasingly important for the modern industry. In design of offshore structures, account is taken of accidental loads such as dropped objects,

\footnotetext{
*Corresponding author. Structural Impact Laboratory, Department of Structural Engineering, Norwegian University of Science and Technology, N-7491 Trondheim, Norway. Tel.: +47-73-59-46-47; fax: +47-73-59-47-01.

E-mail address: tore.borvik@ bygg.ntnu.no (T. Børvik).
} 


\section{Nomenclature}

$b \quad$ target thickness (Bai-Johnson model)

C strain rate sensitivity constant

d diameter

$D \quad$ projectile nose diameter

E Young's modulus

$E_{\mathrm{t}} \quad$ tangent modulus

$h \quad$ thickness

$m$ mass

$p \quad$ depth of penetration

POI point of impact

$r \quad$ radius

$R_{\mathrm{c}} \quad$ hardness Rockwell C

$t \quad$ time

$v \quad$ velocity

$w$ deformation

$\Delta D \quad$ projectile nose deformation, i.e. $\Delta D=D_{\mathrm{f}}-D_{\mathrm{i}}$

$\Delta L \quad$ projectile length reduction, i.e. $\Delta L=L_{\mathrm{i}}-L_{\mathrm{f}}$

Greek letters

$\mu \quad$ friction coefficient

$\theta \quad$ impact angle

$\varphi \quad$ oblique

$\alpha \quad$ pitch

$\gamma \quad$ total yaw

$\beta \quad$ yaw

$\varepsilon \quad$ strain

$\sigma \quad$ stress

\section{Subscripts}

$\begin{array}{ll}0 & \text { initial or flow } \\ \text { bl } & \text { ballistic limit } \\ \text { cr } & \text { critical } \\ \text { c } & \text { cavity } \\ \text { d } & \text { dynamic } \\ \text { e } & \text { engineering value } \\ \text { f } & \text { final or failure } \\ \text { fr } & \text { front side value } \\ \text { i } & \text { initial } \\ \text { max } & \text { maximum value } \\ \text { p } & \text { projectile }\end{array}$




\begin{tabular}{|ll|}
\hline $\mathrm{pl}$ & plug \\
$\mathrm{r}$ & residual \\
$\mathrm{re}$ & rear side value \\
$\mathrm{t}$ & target or true value \\
$\mathrm{u}$ & ultimate \\
$\mathrm{y}$ & yield \\
\hline
\end{tabular}

collisions, explosions and penetration by fragments. Most of these accidental loads are also pertinent in design of protective structures in the process industry or fortification installations for defence purposes. In the transport industry, energy absorption and crashworthiness are now critical issues in the design process of vehicles, vessels and aircrafts. In addition, many of the problems found in structural impact are also relevant in metal forming operations. While much of the work carried out by military and industrial research organisations has been classified, many generic investigations are available in the open literature. As pointed out by Goldsmith [1], the enormous literature on the subject encompasses a variety of different target materials and thicknesses, as well as a host of projectile geometries and a velocity range from about $5 \mathrm{~m} / \mathrm{s}$ to the hypervelocity domain of up to $15 \mathrm{~km} / \mathrm{s}$. Comprehensive reviews on the research into the penetration and perforation of structures by free-flying projectiles can be found in the journal papers by Backman and Goldsmith [2] and Corbett et al. [3], and in the books by Zukas et al. $[4,5]$.

Although many investigations have been presented over the years, the number of papers giving detailed information from ballistic penetration experiments is limited, and the majority of the tests reported are used to validate accompanied theories and models [3]. Several parameters, such as the ratio of target thickness to projectile diameter, the projectile nose shape and the materials involved, are known to affect the penetration mechanisms. Even so, experimental investigations can roughly be divided into three major categories related to the impact velocity of the projectile. The first category covers low velocity impact $\left(v_{\mathrm{i}}<50 \mathrm{~m} / \mathrm{s}\right)$, where target plates normally are hit by heavy projectiles using a drop hammer or a pneumatic accelerator. Work in the area has been reported by Wen and Jones [6], Langseth and Larsen [7-9] and Langseth et al. [10]. The second category covers the other extreme that is high velocity penetration $\left(v_{\mathrm{i}}>1300 \mathrm{~m} / \mathrm{s}\right)$. Here, powder guns, two-stage light gas guns and similar equipment are used to launch low-mass projectiles often against massive target structures. Typical work in this area can be found in special issues of the International Journal of Impact Engineering containing the proceedings from the Hypervelocity Impact Symposium (such as [11]). The last category covers penetration and perforation in the subordnance and ordnance velocity regime $\left(50<v_{\mathrm{i}}<1300 \mathrm{~m} / \mathrm{s}\right)$, where projectiles of arbitrary mass and shape are launched from compressed gas guns or powder guns. This category has received much attention, and particularly those problems dealing with normal impact of plated metallic structures. Some important contributions in this particular field were listed in a paper by Børvik et al. [12], and will not be repeated here.

The complexity of the penetration problem, especially if dynamic material behaviour and fracture are taken into account, has limited the use of closed-form analytical solutions. For that reason, work in this area has been considered as experimental in nature [5]. Even so, a large 
number of theoretical studies have been presented in the literature over the years. The theoretical approaches seem to fall into one of the following categories: (1) Empirical equations based on curve fits to experimental results, (2) analytical models giving simplified solutions to the problem by assuming certain predominant physical effects and (3) numerical simulations where the conservation equations are integrated over successive time increments at all points in an element mesh. However, in recent review papers by Goldsmith [1], Scheffler and Zukas [13] and Zukas and Scheffler [14] questions have been asked about the accuracy of the many computational models available in the literature. It is therefore necessary to validate such models against reliable experimental data.

This paper is divided into three major parts. First, experimental details from almost 100 projectile impact tests are given. In the tests, blunt projectiles were used to impact Weldox $460 \mathrm{E}$ steel plates clamped in a circular frame. The only variables during the tests were the initial projectile velocity and the target plate thickness. A description of the experimental set-up, instrumentation and the materials involved are also given together with an evaluation of possible experimental errors. In the second part, typical empirical equations and analytical models are compared in calculations of the penetration problem. Finally, the last part covers finite element simulations. It is shown that especially the results obtained by the numerical approach are very encouraging in terms of predicting the response of the plates examined.

\section{Experimental programmes}

The experimental set-up, instrumentation and measuring methods used in the tests have partly been presented elsewhere [15,12] and only a summary of the most important features of the equipment is given below. An evaluation of possible errors during a test is also presented. In the tests reported here, which were conducted under identical conditions, at least four types of experimental errors and uncertainties have been identified. The first error is due to projectile attitude prior to impact, the second is associated with the instrumentation and measurements of the penetration process, while the third is related to the boundary conditions of the target plate. Finally, errors and uncertainties also arise due to variations in material and geometrical properties.

\subsection{Compressed gas gun}

The compressed gas gun shown in Fig. 1 [15] was used in all tests to launch the projectile. The main components of the gas gun are the 200 bar pressure tank, the purpose-built firing unit for compressed gas, the $10 \mathrm{~m}$ long smooth barrel of calibre $50 \mathrm{~mm}$ and the closed $16 \mathrm{~m}^{3}$ impact chamber. The gas gun is designed to launch a $250 \mathrm{~g}$ projectile/sabot package to a maximum velocity of $1000 \mathrm{~m} / \mathrm{s}$ when helium is used as propellant. The projectile is mounted in the sabot (Fig. 2), and inserted into the rear end of the barrel. Mylar membranes are used to separate the firing section from the pressure tank and barrel. Compressed gas is pumped into the pressure tank and firing unit simultaneously, but with twice as much pressure in the former. At firing, the firing unit is rapidly evacuated. This sudden increase in pressure gradient over the membrane makes them burst, and the projectile is accelerated down the barrel. When the package leaves the muzzle, 


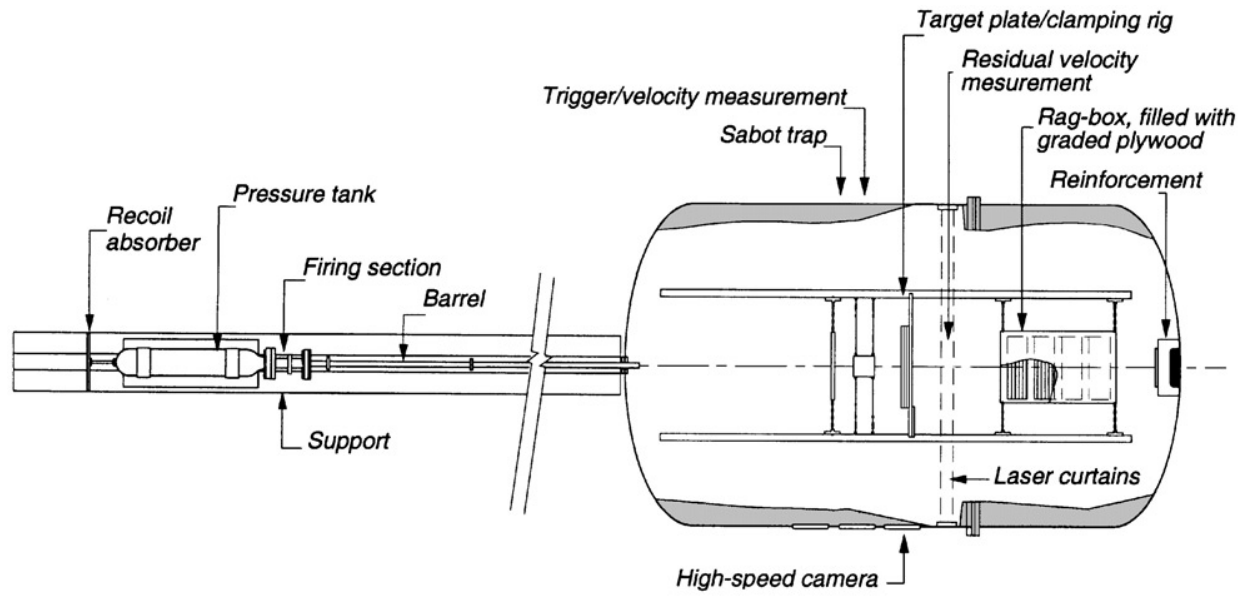

Fig. 1. Sketch of compressed gas gun.

the sabot is immediately separated from the projectile due to aerodynamic forces. A sabot trap, located about $1.5 \mathrm{~m}$ from the muzzle, allows the projectile to pass freely while the sabot parts are stopped. The projectile passes the initial velocity measurement station before it impacts the target clamped in a circular frame after about $2 \mathrm{~m}$ of free flight (Fig. 3). To allow high-speed photography during impact, the clamping system is equipped with a $150 \mathrm{~mm}$ framing window. If the projectile perforates the target, residual velocities are measured before all free flying bodies are stopped without further damage in a rag-box filled with graded plywood. After testing, the impact chamber is opened for final inspections and measurements.

\subsection{Measurements}

To measure initial projectile velocity $\left(v_{\mathrm{i}}\right)$, a photocell system that has two identical light-barriers with LED-light sources on the upper side of the projectile path and detectors on the lower side was used. When the projectile passes between the sources and detectors in the barrier, light is interrupted and signals are given to a digital oscilloscope and a nanosecond counter. The second light-barrier also works as a trigger for the digital high-speed camera system used in the tests. A similar system, shown in Fig. 4, was developed to measure residual velocities if perforation occurs. Here, each light source consists of six lasers mounted in line to give parallel beams in a laser curtain. Detectors are mounted in lines to match the laser light sources. When triggered by a moving body, the detector signals are stored on a $100 \mathrm{MHz}$ digital oscilloscope and information regarding velocity and length of all passing objects is obtained. In all tests, a computer operated Ultranac FS501 digital high-speed camera system was used to photograph the penetration process. The system, which combines an image converter camera capable of taking 200020,000,000 frames per second and a high-resolution CCD camera, was used both for visualisation and measurements. The camera system was set perpendicular to the projectile path in the target plane in order to record the projectile both before and after perforation (see Fig. 3). To reconstruct a $3 \mathrm{D}$ description of the penetration process, mirrors were used in some of the tests. 

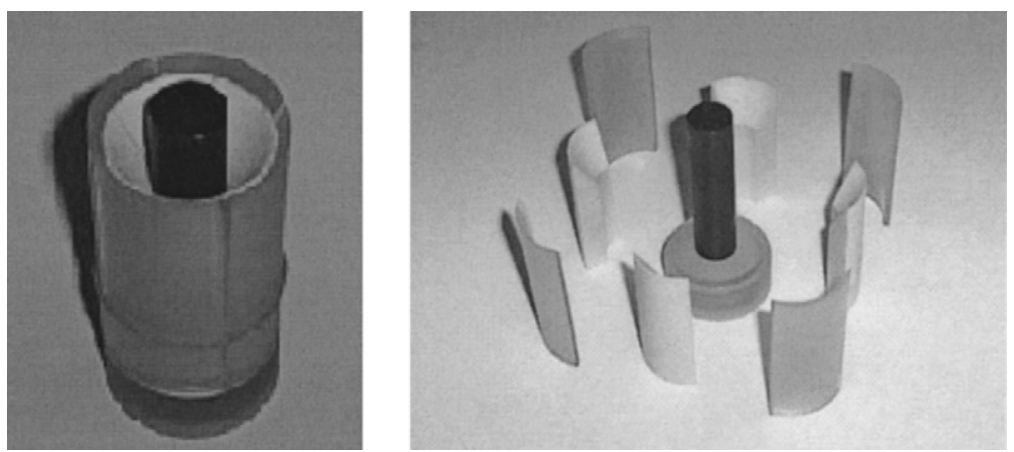

Fig. 2. Projectile sabot design.

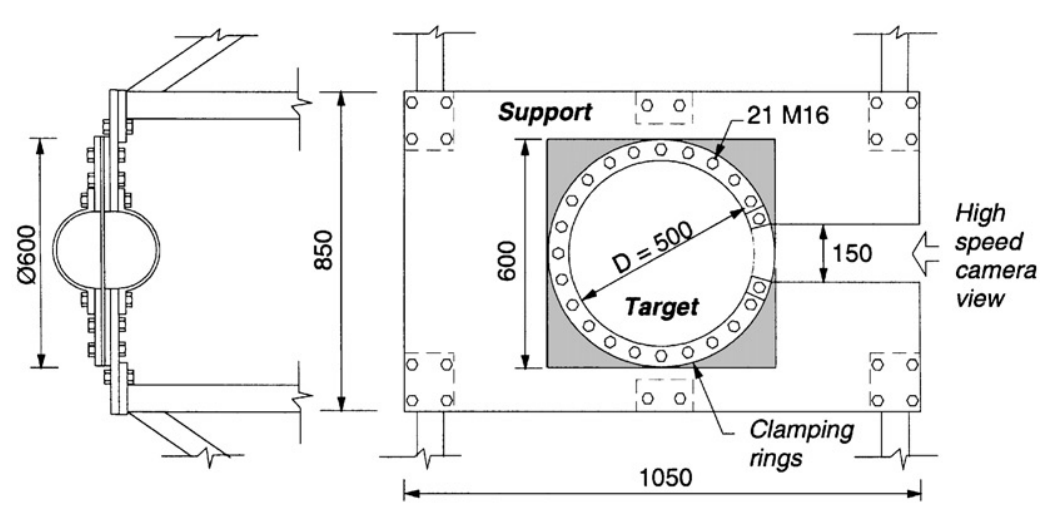

Fig. 3. Target clamped in frame.

These were mounted above the projectile path at an angle of $45^{\circ}$ with respect to the centreline of the camera, one on each side of the target plate. Additional details regarding the gas gun and instrumentation used in this study can be found in $[16,15,12]$.

Measurements of initial and residual velocities are the primary parameters and hence of vital importance in most ballistic impact related experiments. The equipment described above has been purpose-built for this particular study, and it is therefore necessary to estimate the error associated with these measurements. Both the alignment of the equipment and the standard electronics such as counters, lasers and digital oscilloscopes have been evaluated and are believed to be of sufficient accuracy [17]. The largest uncertainty is related to the distribution of LED-light (which gives a larger spread than laser light) over the detectors in the initial velocity measurement station. In order to check this uncertainty, tests were carried out where projectiles were fired through the impact chamber at different velocities without a target plate. The difference in measured velocity between the initial and residual velocity station was found to be less than $1 \%$. Accordingly, measured velocities are supposed to be very close to the actual velocity. The computer sub-routine used to determine the velocity-time curve from the digital high-speed camera images [12] was also checked based on these tests. Due to the sub-pixel resolution, it was 


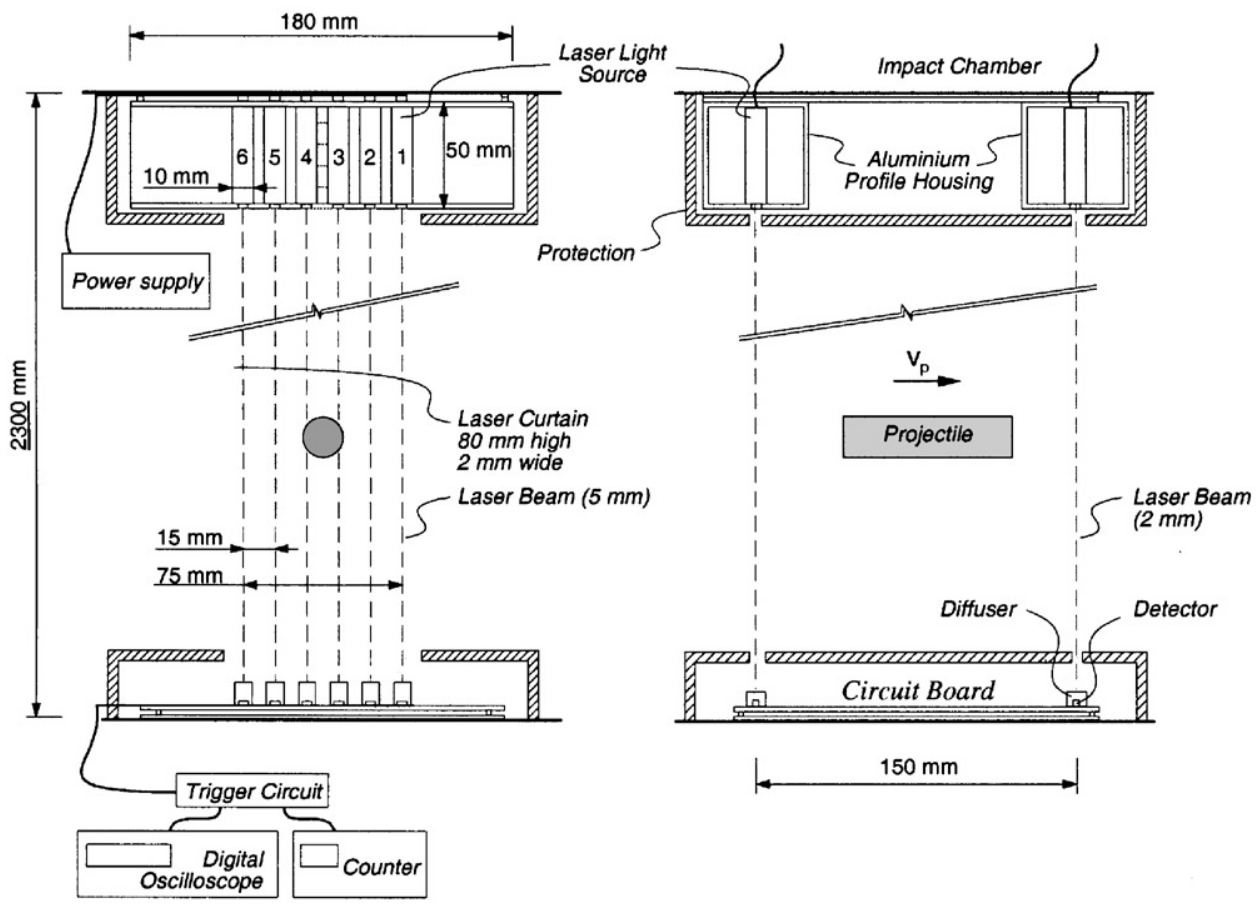

Fig. 4. Residual velocity measurement station.

found that the accuracy in the camera measurements was high, and the deviation was normally within $3-4 \%$ of the velocity measured by the other optical measurement systems.

\subsection{Projectiles and targets}

Blunt-nosed circular-cylindrical projectiles were manufactured from Arne tool steel. After machining, they were oil hardened to a Rockwell C value of $R_{\mathrm{c}}=53\left(\sigma_{\mathrm{y}} \approx 1900 \mathrm{MPa}\right)$ in order to minimise plastic deformation during impact. Nominal hardness $\left(R_{\mathrm{c}} 53\right)$, length $(80 \mathrm{~mm})$, diameter $(20 \mathrm{~mm})$ and mass $(0.197 \mathrm{~kg})$ of the projectile were kept constant in all tests. Before testing, the projectiles were measured, painted dead black and equipped with fiducial marks for high-speed camera measurements. All target plates within a test series were manufactured from the same Weldox 460 E steel plate, having a nominal thickness range from 6 to $30 \mathrm{~mm}$. After careful cutting from the larger plate, 21 equally spaced holes were drilled on a $550 \mathrm{~mm}$ diameter bolt circle before the target was sandblasted on both sides. The plate was finally sandwiched between the clamping rings and bolted to the frame with an inner clamp diameter of $500 \mathrm{~mm}$. Target thickness, initial imperfections, initial oblique and final deformations were measured in situ. The target inserted in the rigid clamping frame is shown in Fig. 3.

A computational model of viscoplasticity and ductile damage for projectile impact has been developed and implemented in the explicit finite element code LS-DYNA [18,19] by Børvik et al. [20]. This model was used to describe the target material behaviour during penetration. Four types of tensile tests are required to identify the material constants in the model. Quasi-static tensile 
tests are used to identify the elastic constants and the yield stress of the material. Notchedspecimen tensile tests define the strain hardening constants, the critical damage and the fracture strain constants. Dynamic tensile tests give the viscoplastic behaviour, while tensile tests at elevated temperatures provide the temperature effect on the stress-strain curve, elastic modulus and fracture strain, respectively. In addition, quasi-static tensile tests were carried out to identify the material constants for the projectile. Engineering mechanical properties for the projectile and target are given in Table 1, while details regarding the material test results and model calibration procedure can be found in [20]. Fig. 5 shows typical quasi-static tensile stress-strain curves both for the target and projectile material. While the target material displays a very ductile behaviour, the projectile is rather brittle. The material information listed in Table 1 is sufficient in order to calibrate the analytical models used in Section 5, while the fully calibrated computational material model of viscoplasticity coupled with ductile damage will be applied in all numerical simulations of the penetration problem presented in Section 6. In the tests, target plates with different thicknesses will be used. This involves material from several deliveries, and scatter in the material properties may appear. In order to reveal such scatter, specimens were machined from 8,10 and $12 \mathrm{~mm}$ thick plates [20]. Coupons were also taken at different locations throughout the thickness (both from the middle and the edge of the plate), and in two different directions $\left(0^{\circ}\right.$ and $\left.90^{\circ}\right)$ with respect to the rolling direction of the target material. The subsequent material tests showed that the scatter in material properties in general was small and is for that reason ignored in the following computations.

Both in analytical and numerical calculations it is assumed that the target is fully clamped at the support, even though such boundary conditions are hard to attain experimentally. This assumption is however reasonable, and it is a general perception that the boundary conditions are of minor importance in ballistic penetration by small mass projectiles in the ordnance velocity regime as long as the target diameter is more than just a few projectile diameters. This was verified through numerical simulations. In the analysis, blunt projectiles were used to perforate 6 and $12 \mathrm{~mm}$ thick plates at impact velocities close to the ballistic limit when the boundary condition varied from fully clamped to simply supported on a stonewall. No significant effect on the residual velocity of the projectile was observed due to the change in clamping conditions. The numerical procedure used in this study is presented in Section 6. The importance of clamping conditions during penetration for this particular problem was also examined in a quasi-static parametric study reported by Børvik and Langseth [21]. From the factorial analysis it was found that the plugging force was unaffected and that only a small effect was noticed on the energy absorbed and maximum target deformation when the boundary condition was changed from fully clamped to simply supported. On the other hand, tests reported by Corran et al. [22] indicate that the clamping force may have a significant effect on the ballistic limit of thin target plates.

Table 1

Quasi-static mechanical properties for the target and projectile [20]

\begin{tabular}{llcccl}
\hline Material & $E(\mathrm{MPa})$ & $\sigma_{\mathrm{y}}(\mathrm{MPa})$ & $\sigma_{\mathrm{u}}(\mathrm{MPa})$ & $E_{\mathrm{t}}(\mathrm{MPa})$ & $\varepsilon_{\mathrm{fe}}$ \\
\hline Target & 200,000 & 490 & 580 & 450 & 0.35 \\
Projectile & 204,000 & 1900 & 2080 & 15,000 & 0.024 \\
\hline
\end{tabular}



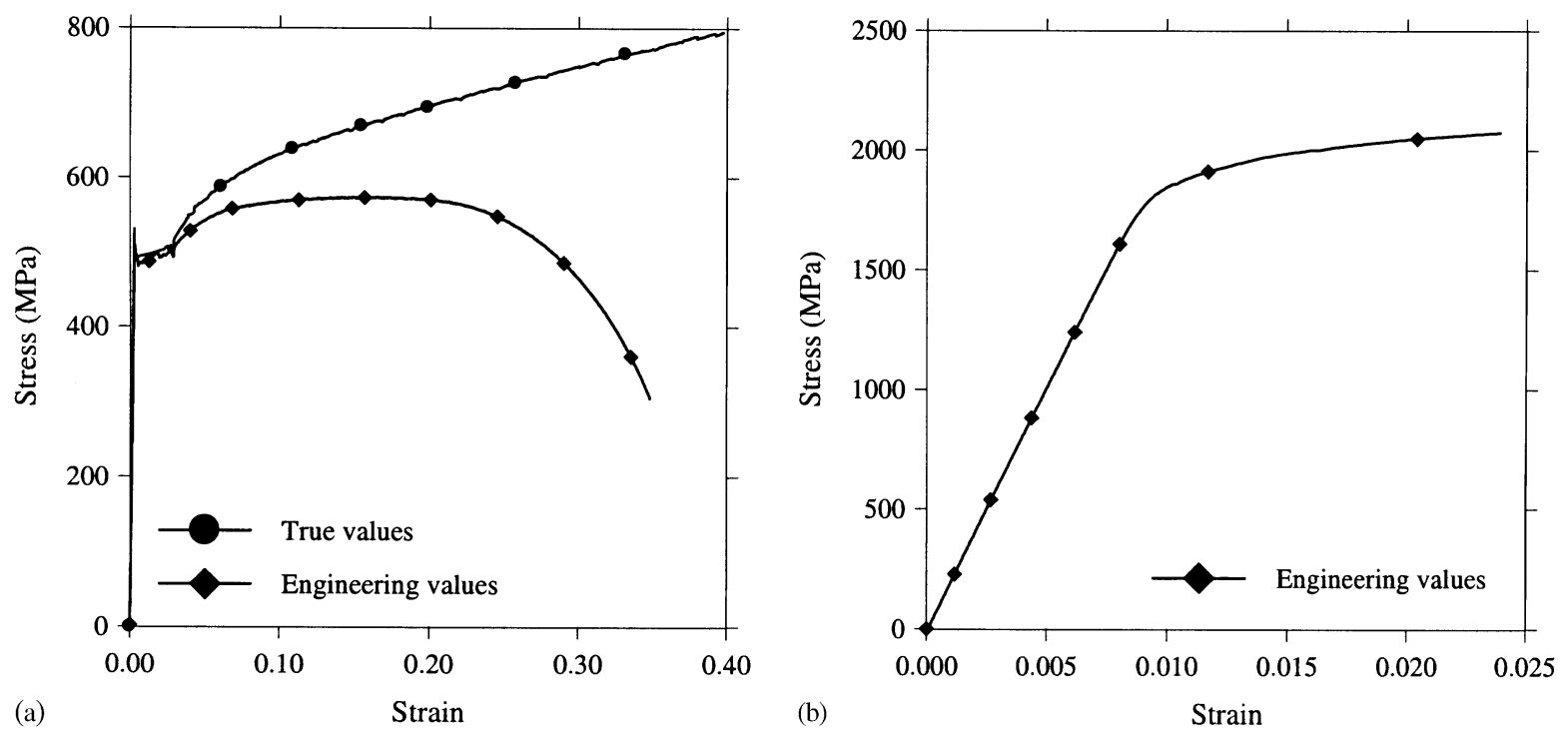

Fig. 5. Typical quasi-static tensile stress-strain curves for (a) Weldox 460 E (target material) and (b) hardened Arne tool steel (projectile material).

\subsection{Exterior ballistics}

Most investigations in penetration mechanics consider normal impact by rigid projectiles on stationary targets, where the velocity vector of the projectile is parallel to its axis of symmetry and normal to the target plane at the point of contact. However, such idealised situations never appear in practice, and some pitch, yaw, oblique and tumbling of the projectile due to aerodynamic forces and instabilities will always be present, as exemplified in Fig. 6. In the figure, the subscripts $0, \mathrm{f}$ and $\mathrm{p}$ refer to initial, final and plug, respectively. An extensive review on the subject of non-ideal projectile impact on targets can be found in [1]. Before impact the attitude of a cylindrical projectile in flight is completely described by a single parameter called total yaw, $\gamma$, and defined as the angle from the velocity vector of the projectile's centre of gravity to the longitudinal axis of the projectile. Total yaw is usually determined from two orthogonal attitude components measured in a ballistic range, called pitch, $\alpha$, if in the vertical plane and yaw, $\beta$, if in the horizontal plane, and given as $\gamma=\tan ^{-1}\left(\tan ^{2} \alpha+\tan ^{2} \beta\right)^{1 / 2}$. The oblique angle, $\varphi$, is the angle from the target normal to the projectile velocity vector and the impact angle, $\theta=\gamma-|\varphi|$, is the angle from the target normal to the symmetry axis of the projectile. As seen, the impact angle will be equal to the yaw angle when the oblique angle is zero. Tumbling impact, denoted by $\omega$, incorporates yaw and oblique as well as an angular motion about an axis through the centre of gravity orthogonal to the longitudinal axis of the projectile. Even if a number of theoretical and experimental investigations have been reported on the subject, only a few have considered the effect of projectile penetration with yaw. In contrast to oblique, yaw is very hard to control in the laboratory because small yaw angles may be required for projectile trajectory stability. However, from the experimental results reported by Goldsmith [1], it appears that the penetration process is hardly affected by total yaw angles up to $3-5^{\circ}$. Larger yaw angles may, on the other hand, significantly reduce the penetration 
capacity of the projectile and have to be taken into consideration. Therefore, in a ballistic experiment it is important to be able to measure projectile attitude prior to and during impact in order to check if the total yaw are small enough to be neglected.

One major disadvantage with the experimental set-up used herein is that the diameter of the barrel is relatively large compared to the diameter of the projectile, and a sabot for guidance and protection of the projectile is required. This sabot has to be completely removed and stopped prior to impact with the target. However, the diameter difference makes massive sabots rather heavy, and tests have revealed that heavy sabots do not separate properly. This has led to the development of the nine-piece serrated sabot system shown in Fig. 2, manufactured from a combination of polycarbonate and foam. The weight of this particular sabot is less than $50 \%$ of the projectile, which was found as a critical value to avoid additional pitch and yaw. It was also learned from [23] that the last meter of the barrel must be as smooth and straight as possible to avoid problems with the sabot separation. Separation of the sabot during flight as the projectile passes the sabot trap is shown in the shadowgraphs presented in Fig. 7. These images also revealed that the originally present titanium plate in the sabot, used to disperse the load from the projectile on the pusher during acceleration, should be removed as it went through the sabot trap and interfered with the projectile.

A method described in [15] was developed for angle measurements. The high-speed camera was used to record the projectile path both before and after perforation and mirrors were used to give a 3D description. An example where this technique was utilised is given in Fig. 12(c), showing perforation of a $10 \mathrm{~mm}$ thick plate both in the vertical (lower part of the image) and horizontal (from the mirrors in the upper part of the image) planes. A computer sub-routine was developed and used to determine both pitch and yaw angles prior to impact based on the digital images. However, the high-speed camera system is a very complex electro-optical instrument. The image converter process, the non-symmetrical optics and the alignment of both the camera and in

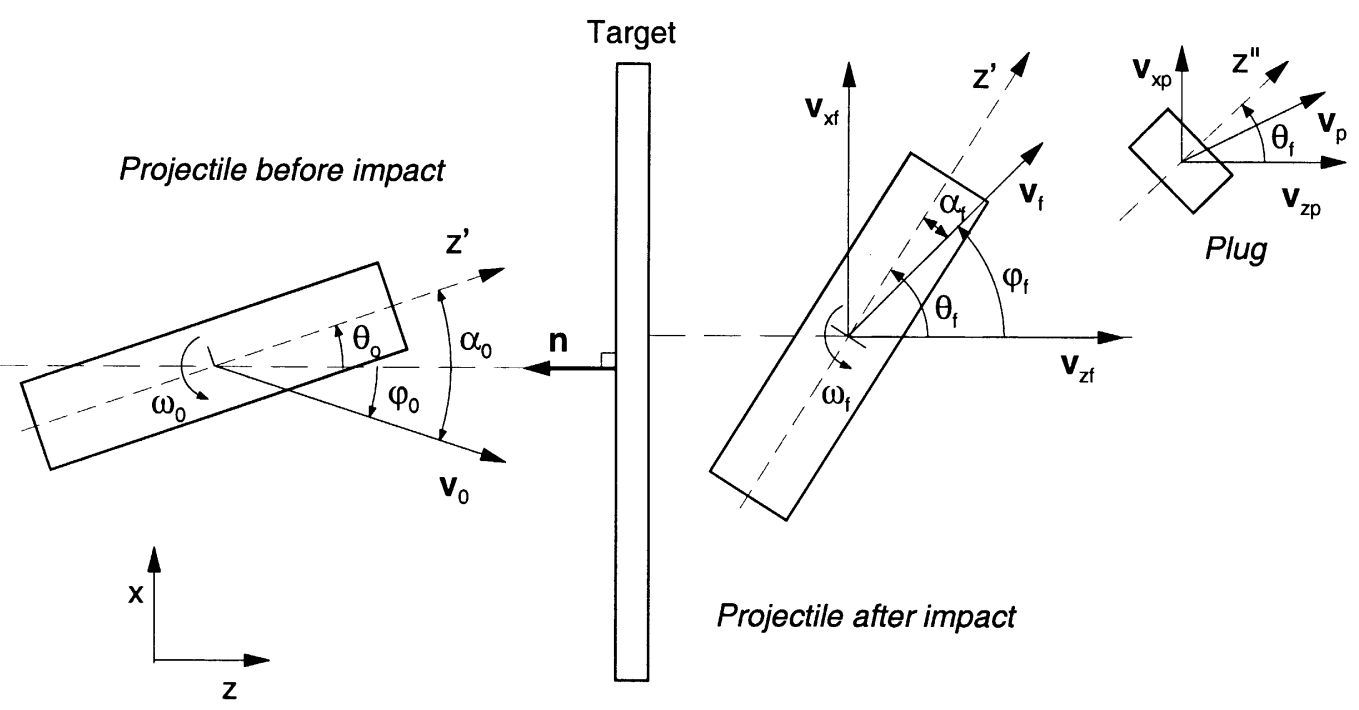

Fig. 6. Definition of projectile attitude during perforation (vertical plane). 

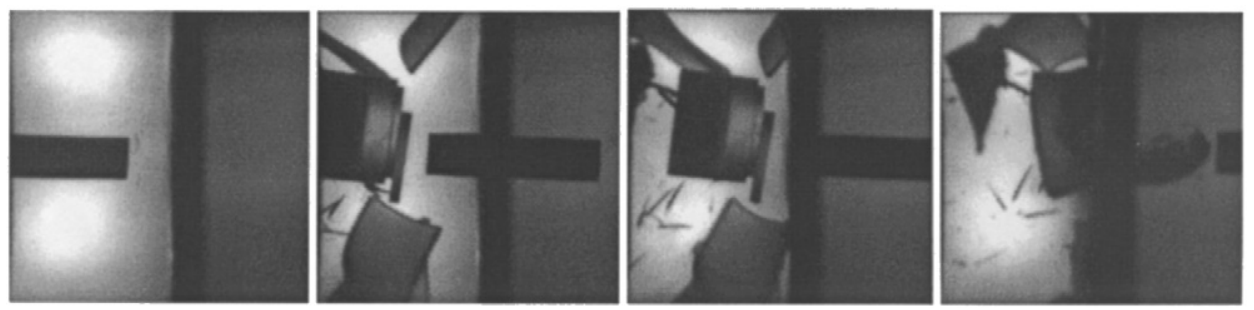

Fig. 7. Projectile passage through the sabot trap, while the separated sabot pieces are stopped.

particular the mirrors cause aberrations and distortions in the images. A spatial calibration of the camera system was thus recommended to achieve reliable measurements. One possible spatial calibration routine is given in $[16,15]$. Unfortunately, the procedure is very time consuming and was therefore only accomplished for the $10 \mathrm{~mm}$ and for some of the $12 \mathrm{~mm}$ thick target tests. It was however revealed from these images that both pitch and yaw were small and well below the critical value. In general, it was noticed that when the pitch was small, so was the yaw. Based on these results, it was assumed that for control reasons it was sufficient to measure the pitch angle only. A simplified method, where the pitch angle of the moving projectile is measured and related to the angle of the stationary support precisely aligned prior to the test, was used in all other tests. In order to measure initial oblique, a collimated laser was inserted in the muzzle of the gun and a mirror was fixed on the target plate surface. The deviation of the reflected laser beam from the centre of the muzzle was measured, and this was used together with the known distance from the laser head to the target plate surface to calculate the oblique angle of the target. Measured pitch, yaw and oblique angles are given in Table 2. As seen, the angle deviations are small and a normal impact can be assumed in these tests.

\section{Experimental results}

In the present experimental study, the parameters varied during the tests are the initial projectile velocity and the nominal target thickness. The effect on the structural response when the impact velocity is varied, while keeping the target thickness constant at $r_{\mathrm{p}} / h_{\mathrm{t}} \sim 1$, has been investigated earlier [12]. Some observations from that particular study are repeated below.

- The target is sensitive to variations in impact velocity near the ballistic limit, and the residual velocity of the projectile is therefore never really small. Thus, the residual versus impact velocity curve exhibits an almost discontinuous "jump" at the ballistic limit velocity. As the impact velocity is increased, the curve approaches the ballistic limit line, i.e. the ballistic limit velocity for a target with zero thickness. The velocity of the plug is always found to be higher than that of the projectile.

- The amount of projectile kinetic energy converted into projectile/target work decreases with increasing impact velocity. At the highest impact velocities, the amount of energy absorbed by the projectile/target system flattens out and becomes close to constant.

- The target deformation consists of a combination of localised bulging and global dishing, which becomes more and more localised as the impact velocity is increased. 


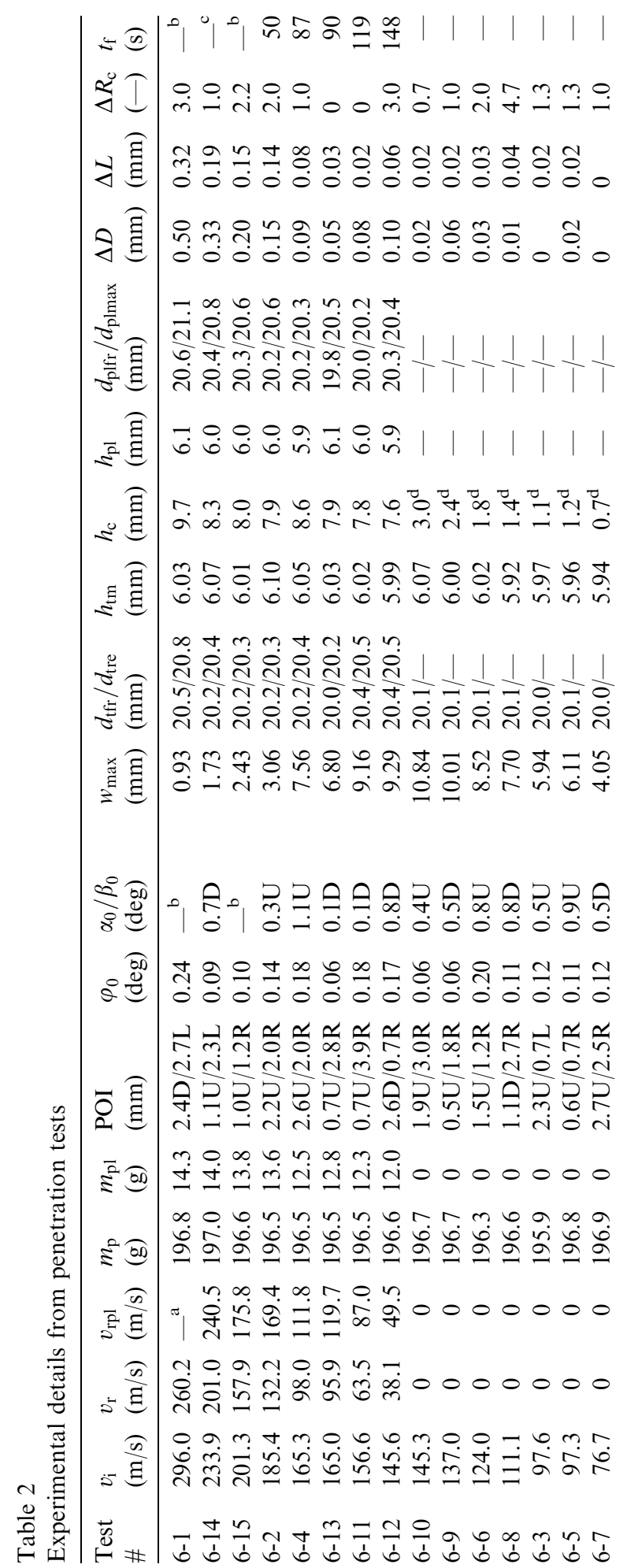

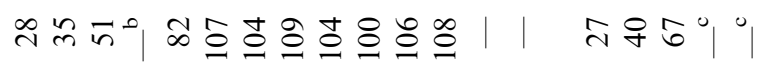

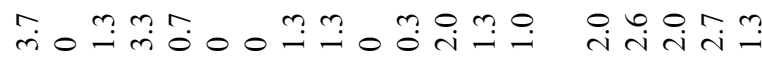

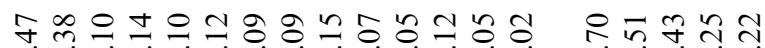

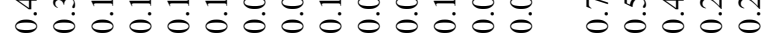

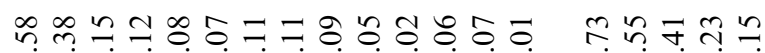

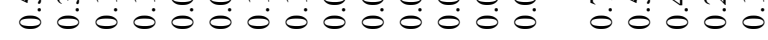

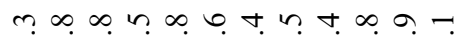

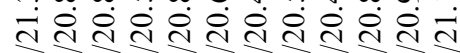

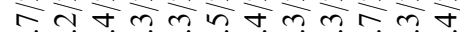
ठิंतेंतेंतेत्तेंतेंते

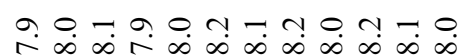

in $\ln x$ in

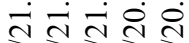
$\infty 6 m$ 两 तेत्तें

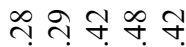
नेंबन

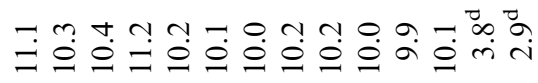

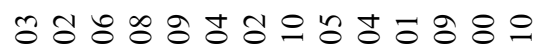

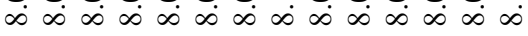

N

ते तेंतेंतेंतेंते ते

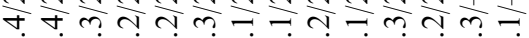

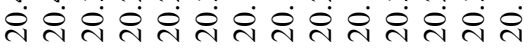

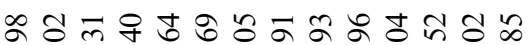

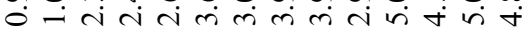

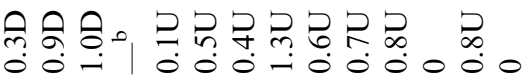
तู่

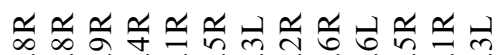

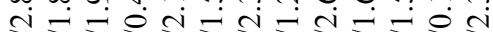
영

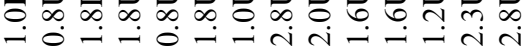

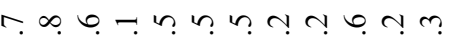
$\infty \infty \dot{\infty} \infty \infty \infty \infty \infty \infty \infty$ $\infty \infty$ n $\infty$ m ๖े ก ก ก t

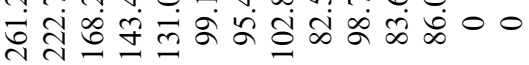
trmoormonoro fa o. on

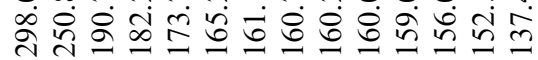

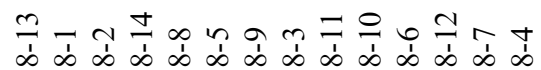

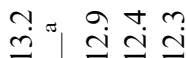
$\infty N N N$ बं

ก. ¿ั่ n t m m m ণ 20500 ○்

$\exists \quad \stackrel{m}{2} \cong$ 它 永寻己主

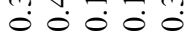

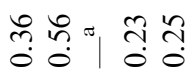

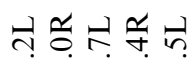
$\dot{\circ} \dot{\circ} \dot{0} 0$ 으승 ते ले ते ठ용 $-4 y-a n$ ส่ส ‥ t. t. m n ㅇํㅇㅇำ m. 0.0.

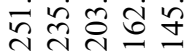
na

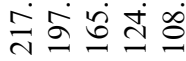
o n n 0 .

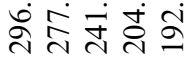

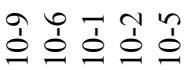




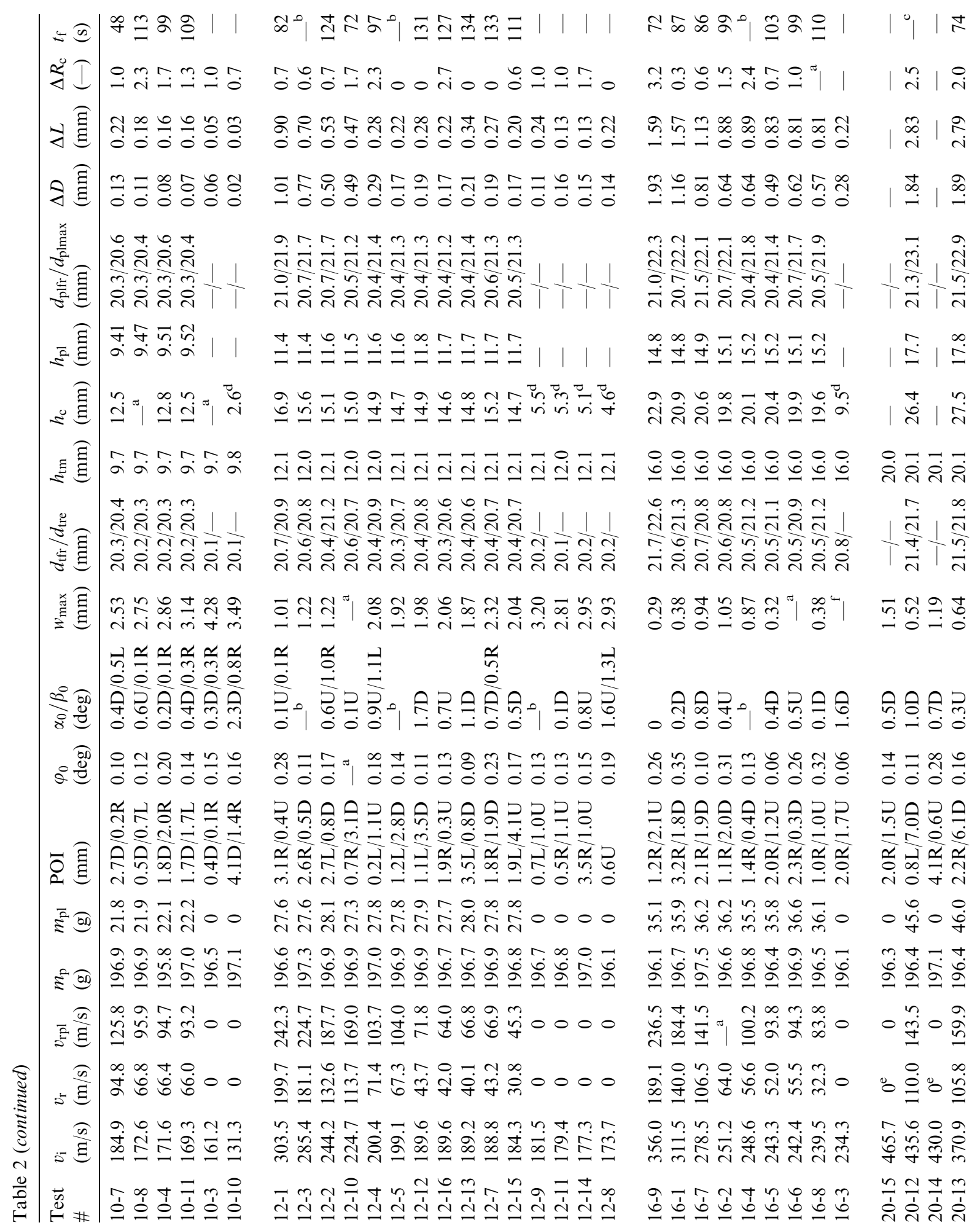




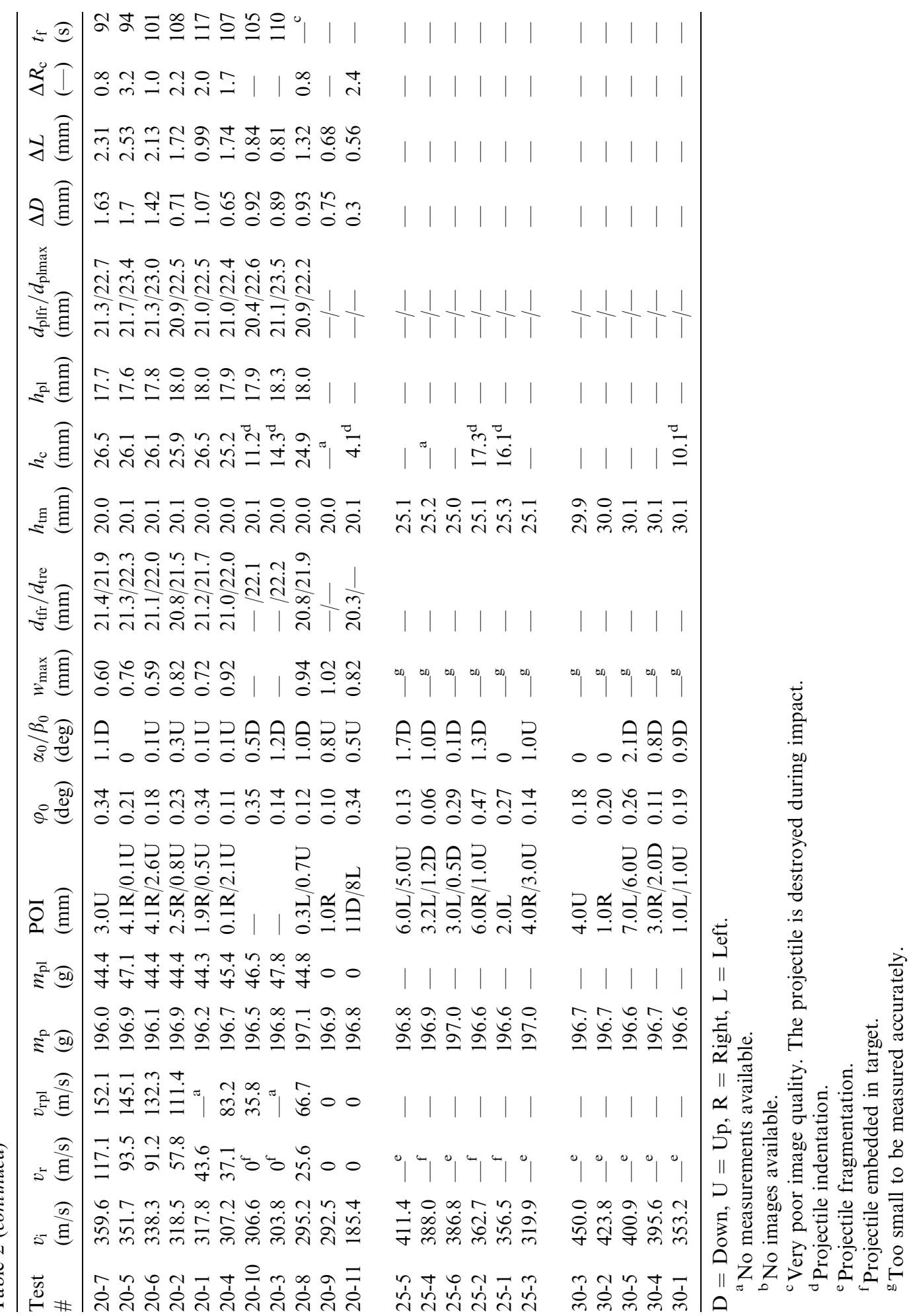


- The permanent target deformation increases with increasing projectile velocity until it reaches a maximum near the ballistic limit. After perforation, the deformation decreases with increasing velocity until it stabilises at a velocity well above the ballistic limit. The maximum deformation during a test is about twice as big as the permanent one.

- The projectile plastic deformation increases with increasing impact velocity. Also the plug thinning, i.e. the difference between the initial and final thickness of the plug, shows an increase with increasing projectile velocity.

Depending on the specific impact configuration, such as target thickness or material and projectile nose shape, various modes of failure may occur during penetration. Backman and Goldsmith [2] classified these as fracture due to an initial stress wave, radial fracture behind an initial wave in brittle materials, spalling or scabbing, fragmentation, plugging, petaling and ductile hole enlargement. In perforation of metal plates by blunt projectiles, plugging and ductile hole enlargement seem to be the most distinct failure modes. Plugging, which is a failure mode dominated by simple or adiabatic shear, takes place with almost no lateral compression in the target material due to the localisation of the deformation in narrow shear zones around the sharp edges of a blunt projectile. However, as the target becomes thick, the material in front of the projectile has to be pushed out laterally in order to form a penetration channel. When the thickness of the remaining target ahead of the advancing projectile approaches some limit, the penetration process may change from hole enlargement to plugging, which is a less energy consuming failure mode. This will be discussed in Section 6 . The metallurgical mechanisms associated with shear plug formation and thermoplastic instability were recently investigated by Børvik et al. [24]. This study showed heavily deformed shear bands in 10-16 mm thick plates, while transformed adiabatic shear bands were found in thicker plates. In plates thinner than $10 \mathrm{~mm}$, the plugging process seemed to be controlled by a combination of localised shear, global bending and membrane action.

In this paper focus will be on the observed variation in ballistic resistance when the target response changes as the thickness increases. Experimental details from about 100 impact tests with blunt projectiles at striking velocities between 75 and $500 \mathrm{~m} / \mathrm{s}$ on clamped Weldox $460 \mathrm{E}$ steel plates with thicknesses ranging from 6 to $30 \mathrm{~mm}$ are given in Table 2. The measured initial and residual projectile velocities were used to construct the curves shown in Fig. 8. The solid lines through the data points are calculated based on a model originally proposed by Recht and Ipson [25]

$$
v_{\mathrm{r}}=a\left(v_{\mathrm{i}}^{p}-v_{\mathrm{bl}}^{p}\right)^{1 / p}
$$

where the method of least squares was used to obtain a best fit of the model constants $a$ and $p$ to the experimental data. The ballistic limit velocity $v_{\mathrm{bl}}$ was calculated as the average between the highest projectile velocity not giving perforation and the lowest projectile velocity giving complete perforation of the target. Table 3 gives the ballistic limit velocities for each target thickness tested, together with the fitted constants $a$ and $p$ from Eq. (1). Much effort was spent in the attempt of defining the ballistic limit as closely as possible, and the accuracy in the determination of the different ballistic limit velocity is indicated in Table 3. Note that the stated accuracies only apply to the experimental tests carried out in this study, and does not 


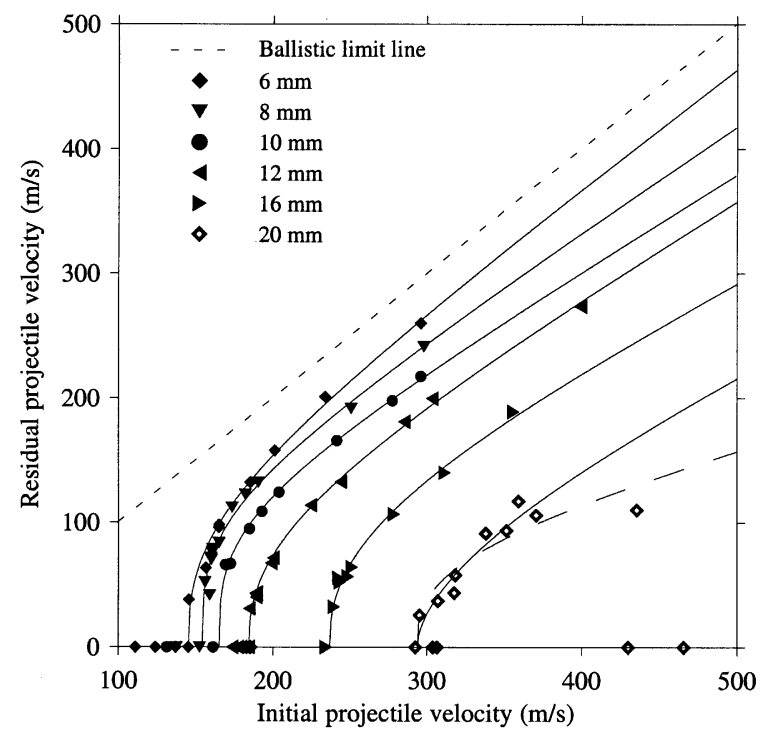

Fig. 8. Experimental residual versus impact velocity curves. The solid lines through the data points are based on a best fit to the Recht-Ipson [25] model. The dashed line through the data points for the $20 \mathrm{~mm}$ thick target is based on a best fit to the Recht-Ipson model where also the rather unsure data point at an impact velocity of $436 \mathrm{~m} / \mathrm{s}$ is included.

Table 3

Experimentally obtained ballistic limit velocities versus target thickness

\begin{tabular}{llllllll}
\hline & \multicolumn{7}{l}{ Target thickness $(\mathrm{mm})$} \\
\cline { 2 - 7 } & 6 & 8 & 10 & 12 & 16 & 20 & $25-30$ \\
\hline$v_{\mathrm{bl}}(\mathrm{m} / \mathrm{s})$ & 145.5 & 154.3 & 165.3 & 184.5 & 236.9 & 293.9 & $-^{\mathrm{a}}$ \\
Accuracy $(\mathrm{m} / \mathrm{s})$ & \pm 0.2 & \pm 1.8 & \pm 4.1 & \pm 0.3 & \pm 2.6 & \pm 1.4 & $-^{\mathrm{a}}$ \\
$a$ & 0.94 & 0.84 & 0.76 & 0.74 & 0.63 & $0.57(0.34)$ & $-^{\mathrm{a}}$ \\
$p$ & 2.71 & 3.30 & 3.23 & 2.42 & 2.40 & $1.78(2.85)$ & $-^{\mathrm{a}}$ \\
\hline
\end{tabular}

${ }^{\text {a }}$ Perforation was not obtained in any of these tests due to projectile fragmentation.

involve any statistical spread as would have been expected if several tests were carried out with identical impact velocities.

Both the "jump" in residual velocity at the ballistic limit and the slope of the residual versus impact velocity curve seem to decrease with target thickness and initial projectile velocity. Forrestal and Hanchak [26] predicted a similar distinct jump in perforation of thin plates. The height of the jump is believed controlled by the amount of tension at the rear side of the target close to failure. The reduced slope of the residual versus impact velocity curves in Fig. 8 with increased target thickness is probably caused by an increased plastic deformation of the projectile with increased impact velocity and target thickness, as will be discussed later. For targets with thicknesses of $20 \mathrm{~mm}$ or more, the projectile was severely damaged. The projectile shattered at impact in most tests using 25 or $30 \mathrm{~mm}$ thick targets, independent of initial velocity (Table 2), and 


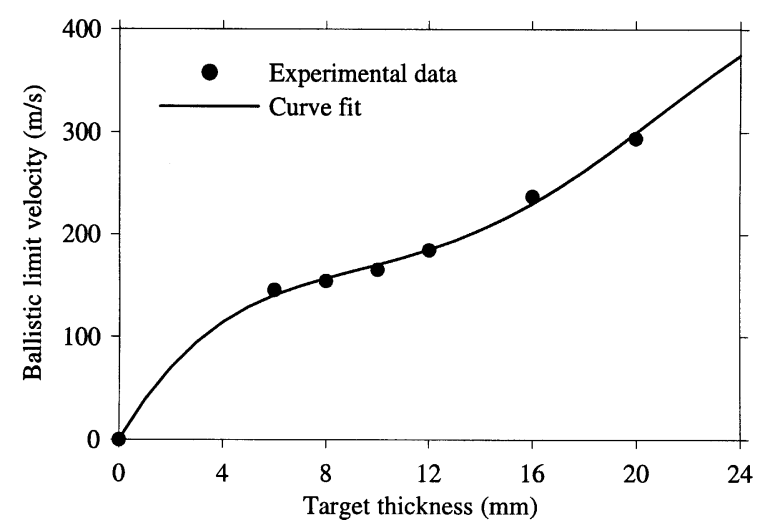

Fig. 9. Ballistic limit velocity versus target thickness.

it was thus not possible to obtain the ballistic limit velocity or residual versus impact velocity curve for these two thicknesses (see Table 3). The projectile also shattered at the highest impact velocities in the $20 \mathrm{~mm}$ thick target, and the scatter in the experimental results became considerable. For that reason, two curves were fitted to the data points. In the first curve, given as the solid line in Fig. 8, only data from tests at impact velocities below $400 \mathrm{~m} / \mathrm{s}$ were used in the fit. In the second curve, i.e. the dashed line in Fig. 8, also the data point at an impact velocity of 436 $\mathrm{m} / \mathrm{s}$ was included. In that particular test, the projectile was more affected by plastic deformation and damage than the other results, and this is probably the reason for the relatively low residual velocity.

Fig. 9 shows experimentally obtained ballistic limit velocities versus target thickness. As seen, a distinct kink appears in the fitted response curve at a target thickness of about $10 \mathrm{~mm}$. This shift in target response appears to be related to the change in deformation mode from typical thin plate membrane stretching towards thick plate shear localisation. Corran et al. [22] obtained a similar behaviour in penetration tests on steel and aluminium target plates. The effect of this difference in target response during impact could be important. From Fig. 9 it is seen that a doubling of the target thickness from e.g. 6 to $12 \mathrm{~mm}$ increases the ballistic limit of the target by less than $30 \%$. In situ measured maximum target deformations are given in Fig. 10. While the permanent deformation is almost twice the target thickness for a $6 \mathrm{~mm}$ target, it is hardly measurable for a $16 \mathrm{~mm}$ thick plate. In thin plates, both localised bulging and global structural deformation take place. It is assumed that the global deformation mode absorbs a considerable amount of projectile kinetic energy during the impact [27]. In thick plates hardly any global target deformation is measured and most of the projectile kinetic energy has to be absorbed in the highly localised shear zones surrounding the projectile. This is further illustrated in Fig. 11, where in situ permanent deformation profiles for different target thicknesses at an impact velocity close to the respective ballistic limit are shown. Note that no plastic deformation could be measured at the boundary in any of these tests. Target deformation is not a function of plate thickness alone. Impact velocity is also crucial and the deformation reaches a maximum near the ballistic limit independent of target thickness. This was also shown in [12] where permanent deformation profiles as a function of impact velocity for $12 \mathrm{~mm}$ thick targets were plotted and compared. 


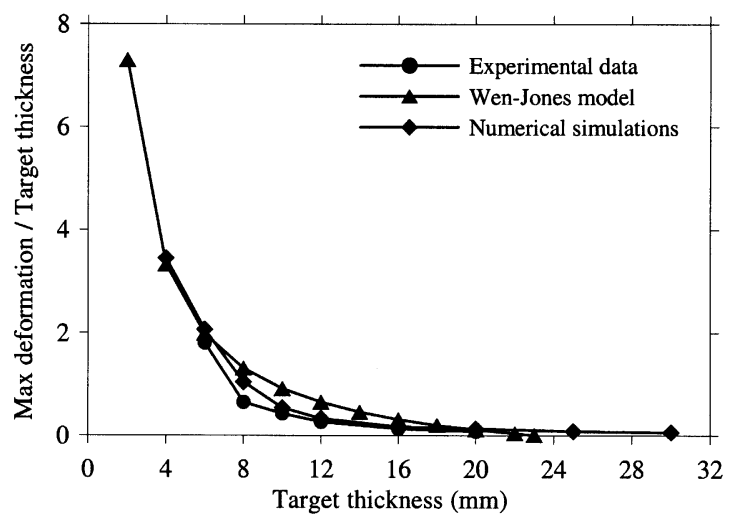

Fig. 10. Relative maximum target deformation versus target thickness.

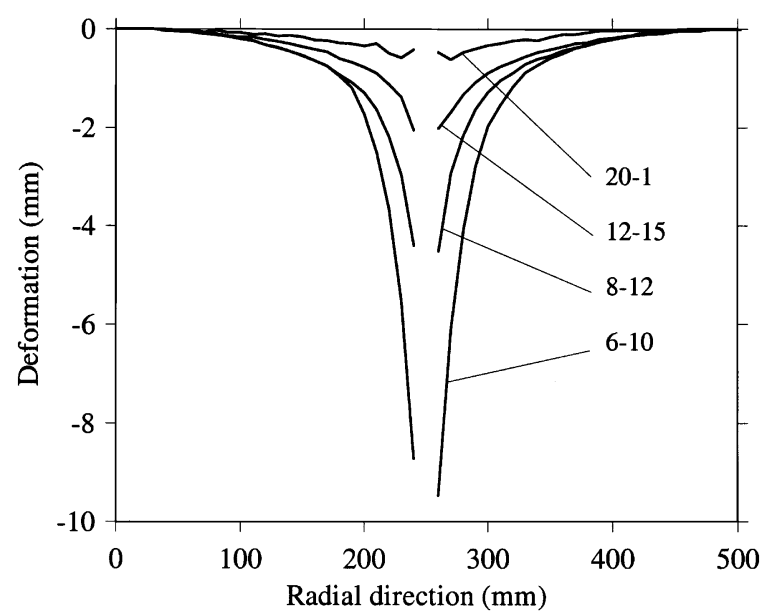

Fig. 11. Measured permanent target deformation profiles at impact velocities close to the ballistic limits. The first number in the legend indicates the nominal target thickness, while the second number indicates the test number (see Table 2 for more experimental details).

High-speed camera images from tests at impact velocities close to the ballistic limits are presented in Fig. 12. As seen, all targets failed by plugging. Fig. 12(a) and (b) show that the global deformation in 6 and $8 \mathrm{~mm}$ thick targets are considerable. The maximum deformation during impact is found to be approximately twice as large as the measured permanent one given in Fig. 10. However, this changes rapidly with target thickness and impact velocity. Fig. 12(c) and (d) show perforation of 10 and $12 \mathrm{~mm}$ thick targets, respectively. In these tests the deformation is much more localised, even if the impact velocity also here is close to the ballistic limit. When the target becomes thick, the deformation becomes extremely localised. This is seen in Fig. 12(e) and (f), where perforation of 16 and $20 \mathrm{~mm}$ thick targets is shown. Here, hardly any global deformation is detected except for the highly localised bulge on the rear side of the target after severe projectile indentation. Note also that due to an unsymmetrical failure mode, the projectile may rotate somewhat in the cavity during penetration even though both pitch and yaw are small. 
(a) Test \# 6-11 ( $\left.\mathrm{v}_{\mathrm{i}}=156.6 \mathrm{~m} / \mathrm{s}, \mathrm{v}_{\mathrm{r}}=63.5 \mathrm{~m} / \mathrm{s}, \mathrm{v}_{\mathrm{rpl}}=87.0 \mathrm{~m} / \mathrm{s}, \mathrm{v}_{\mathrm{i}} / \mathrm{v}_{\mathrm{bl}}=1.076\right)$ :

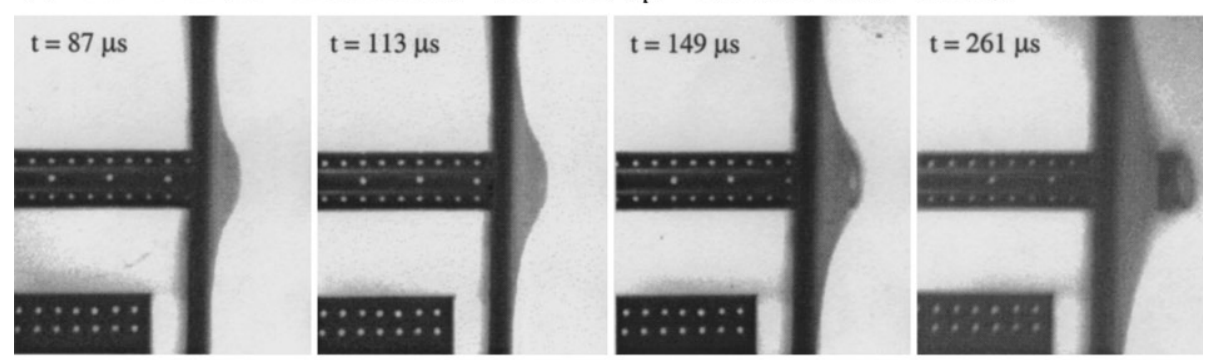

(b) Test \# 8-8 $\left(\mathrm{v}_{\mathrm{i}}=173.7 \mathrm{~m} / \mathrm{s}, \mathrm{v}_{\mathrm{r}}=112.0 \mathrm{~m} / \mathrm{s}, \mathrm{v}_{\mathrm{rpl}}=131.0 \mathrm{~m} / \mathrm{s}, \mathrm{v}_{\mathrm{i}} / \mathrm{v}_{\mathrm{bl}}=1.126\right)$ :

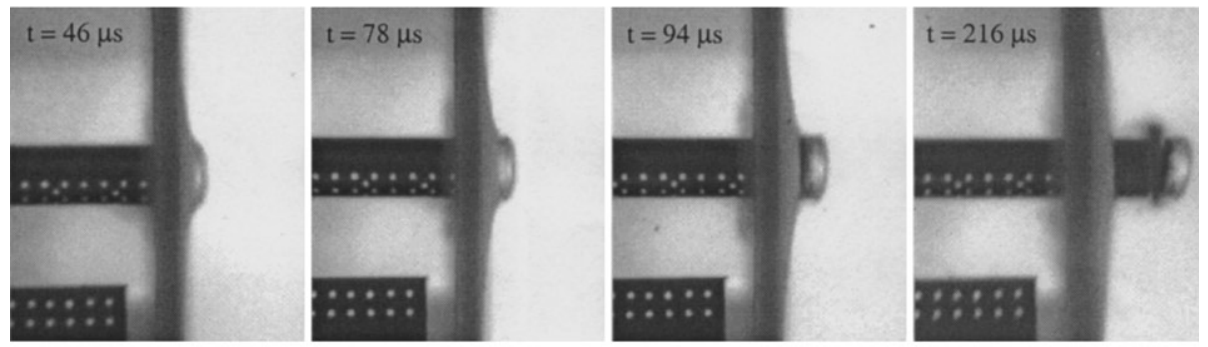

(c) Test \# 10-7 ( $\left.\mathrm{v}_{\mathrm{i}}=184.9 \mathrm{~m} / \mathrm{s}, \mathrm{v}_{\mathrm{r}}=94.8 \mathrm{~m} / \mathrm{s}, \mathrm{v}_{\mathrm{rpl}}=125.8 \mathrm{~m} / \mathrm{s}, \mathrm{v}_{\mathrm{i}} / \mathrm{v}_{\mathrm{bl}}=1.119\right)$ :

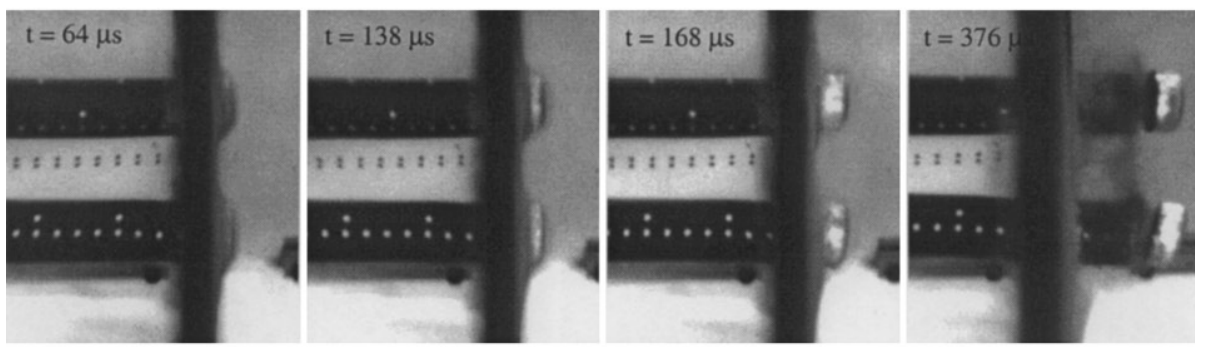

(d) Test \# 12-16 $\left(\mathrm{v}_{\mathrm{i}}=189.6 \mathrm{~m} / \mathrm{s}, \mathrm{v}_{\mathrm{r}}=42.0 \mathrm{~m} / \mathrm{s}, \mathrm{v}_{\mathrm{rpl}}=64.0 \mathrm{~m} / \mathrm{s}, \mathrm{v}_{\mathrm{i}} / \mathrm{v}_{\mathrm{bl}}=1.028\right)$ :
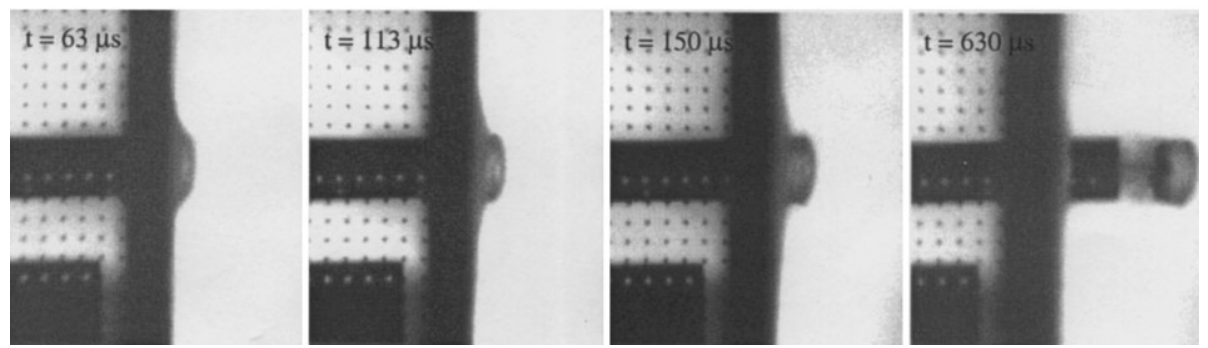

Fig. 12. Some high-speed camera images from tests at impact velocities close to the ballistic limit for different target thicknesses. In all images (except for those in (c)), the stationary reference points used for high-speed camera velocity measurements can be seen in the lower-left corner. In (c), mirrors were used to give a 3D description of the penetration process, and the reference points were placed directly on the front-side mirror. 
(e) Test \# 16-6 $\left(\mathrm{v}_{\mathrm{i}}=242.4 \mathrm{~m} / \mathrm{s}, \mathrm{v}_{\mathrm{r}}=55.5 \mathrm{~m} / \mathrm{s}, \mathrm{v}_{\mathrm{rpl}}=94.3 \mathrm{~m} / \mathrm{s}, \mathrm{v}_{\mathrm{i}} / \mathrm{v}_{\mathrm{bl}}=1.023\right)$ :

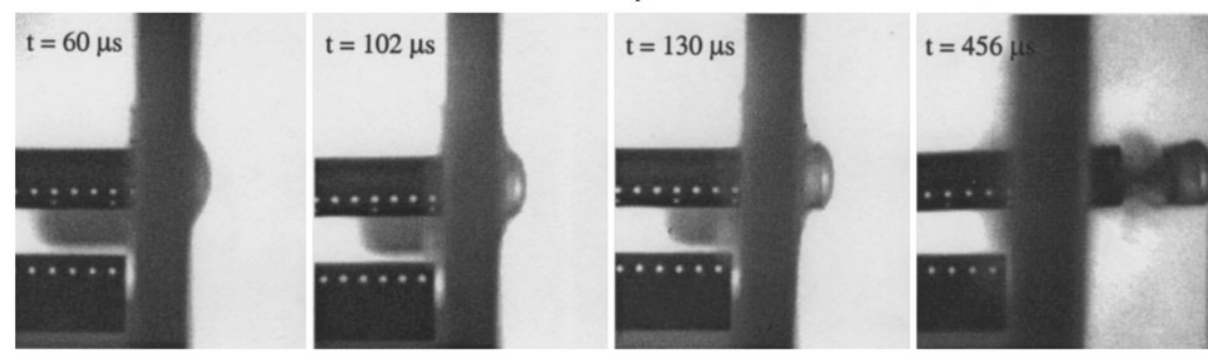

(f) Test \# 20-4 ( $\left.\mathrm{v}_{\mathrm{i}}=307.2 \mathrm{~m} / \mathrm{s}, \mathrm{v}_{\mathrm{r}}=37.1 \mathrm{~m} / \mathrm{s}, \mathrm{v}_{\mathrm{rpl}}=83.2 \mathrm{~m} / \mathrm{s}, \mathrm{v}_{\mathrm{i}} / \mathrm{v}_{\mathrm{bl}}=1.045\right)$ :

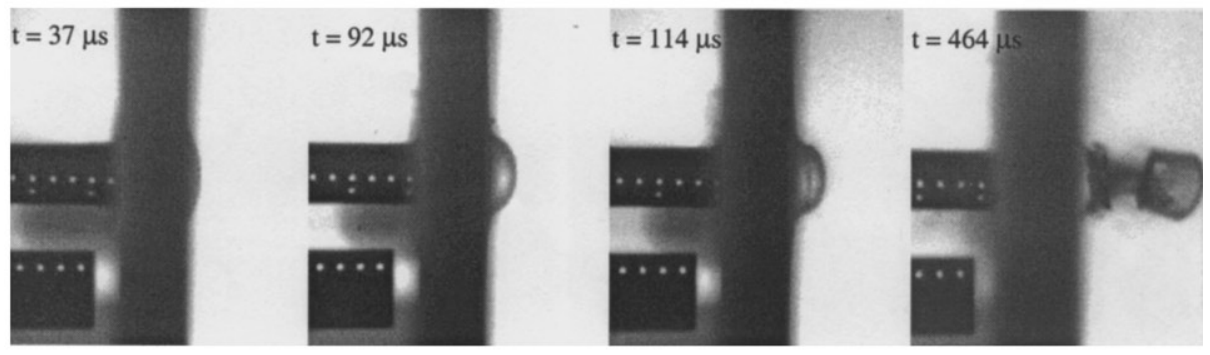

(g) Test \# 25-5 ( $\left.\mathrm{v}_{\mathrm{i}}=411.4 \mathrm{~m} / \mathrm{s}, \mathrm{v}_{\mathrm{r}}=0 \mathrm{~m} / \mathrm{s}, \mathrm{v}_{\mathrm{rpl}}=0 \mathrm{~m} / \mathrm{s}\right)$ :

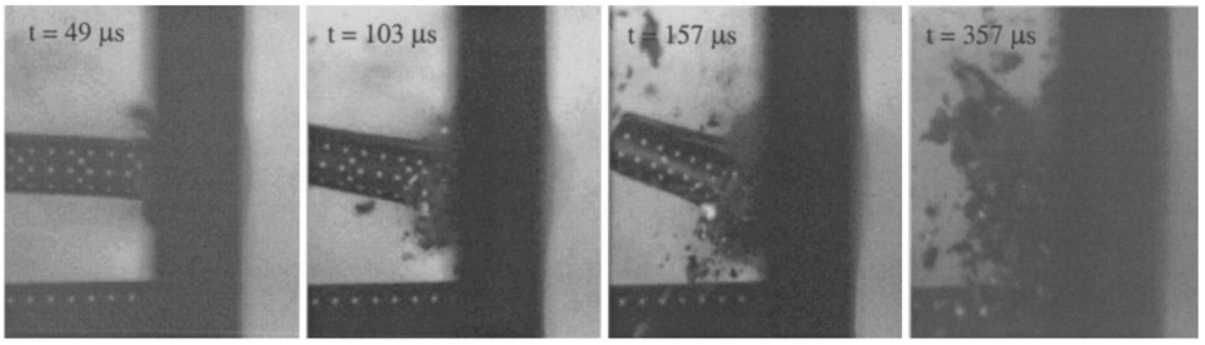

(h) Test \# 30-3 ( $\left.\mathrm{v}_{\mathrm{i}}=452.0 \mathrm{~m} / \mathrm{s}, \mathrm{v}_{\mathrm{r}}=0 \mathrm{~m} / \mathrm{s}, \mathrm{v}_{\mathrm{rpl}}=0 \mathrm{~m} / \mathrm{s}\right)$ :

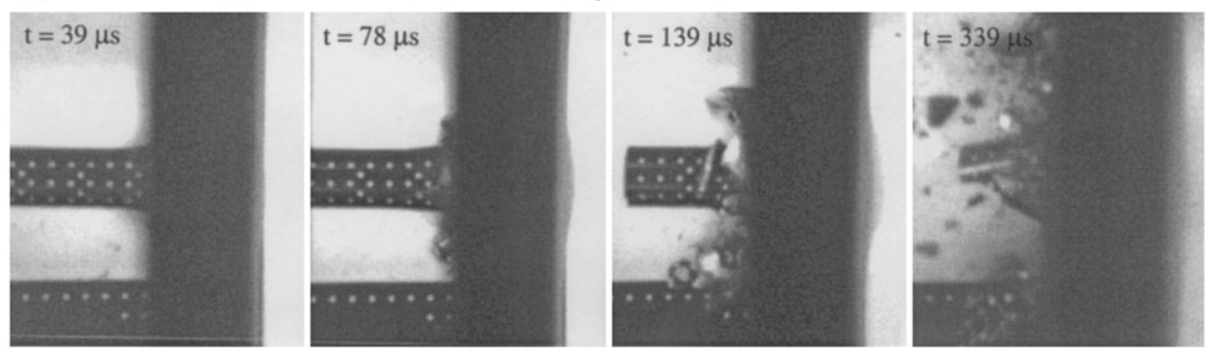

Fig. 12 (continued). 


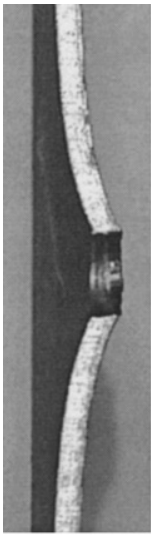

6-11

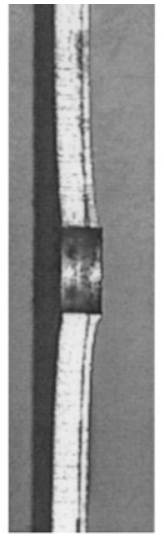

8-8

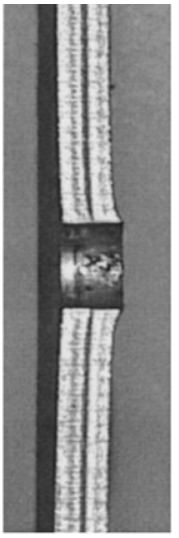

$12-16$

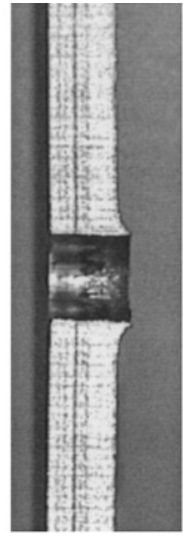

$16-6$

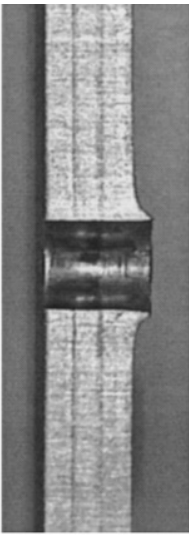

$20-4$

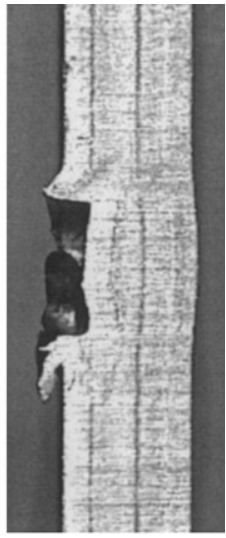

$30-3$

Fig. 13. Cross-sections of perforated and penetrated targets of varying thickness.

For the thickest plates, the projectile is destroyed. Fig. 12(g) and (h) show complete break-up of the brittle projectile (Fig. 5) during impact of a 25 and $30 \mathrm{~mm}$ thick target plate, respectively, with only a limited target deformation on the rear side. It was not possible to perforate any of the 25 or $30 \mathrm{~mm}$ thick targets with the type of projectile used in this study, indicating an upper limit for projectile capacity. As revealed in Fig. 8 and Table 2, the projectile shattered also for the highest impact velocities in $20 \mathrm{~mm}$ thick targets. The reason is the extreme interface force, causing severe circumferential tensile stresses between the projectile nose and the target surface, in blunt projectile penetration of thick plates. This was also found in a study on the importance of projectile nose shape in structural impact [28,29]. As seen from the first images in Fig. 12(g) and (h), the projectile starts to mushroom at impact. However, due to the low fracture strain it is cleaved and completely destroyed during penetration. In an attempt to avoid this complete destruction, several tests were carried out with softer projectiles ( $R_{\mathrm{c}} 48$ and 43). Also these projectiles fractured at impact. If further softened (down to $R_{\mathrm{c}} 30$ ), the plastic deformation of the projectile became severe, which again considerably affected the ballistic capability. Shatter was not observed in any of the tests with a target thickness less than $20 \mathrm{~mm}$. Cross-sections of perforated targets close to the ballistic limit are given in Fig. 13. Again, the global deformation decreases and the rear side bulge increases with increasing target thickness. The cavity is smooth and regular in all tests except for the 25 and $30 \mathrm{~mm}$ thick plates where the projectile fractured. Here, the target damage is very limited even though the impact velocity of the projectile was much higher than for the other tests. Corresponding projectiles and plugs are shown in Fig. 14. The plastic deformation in the projectile is limited at impact velocities close to the ballistic limit for target thicknesses between 6 and $20 \mathrm{~mm}$. Also the plugs are seen to be both smooth and cylindrical. It was noticed that the plugs have a significant bulge on the distal side, and a variable shear zone height was observed on the surface of the plug in some of the tests. In contrast, Fig. 15 shows a completely shattered projectile after impact with a $30 \mathrm{~mm}$ thick target plate.

The velocity-time curve from the penetration experiment is an important quantity both regarding physical understanding and validation of computational models. A computer subroutine [12] was developed in order to measure the relative displacement of the projectile from the 


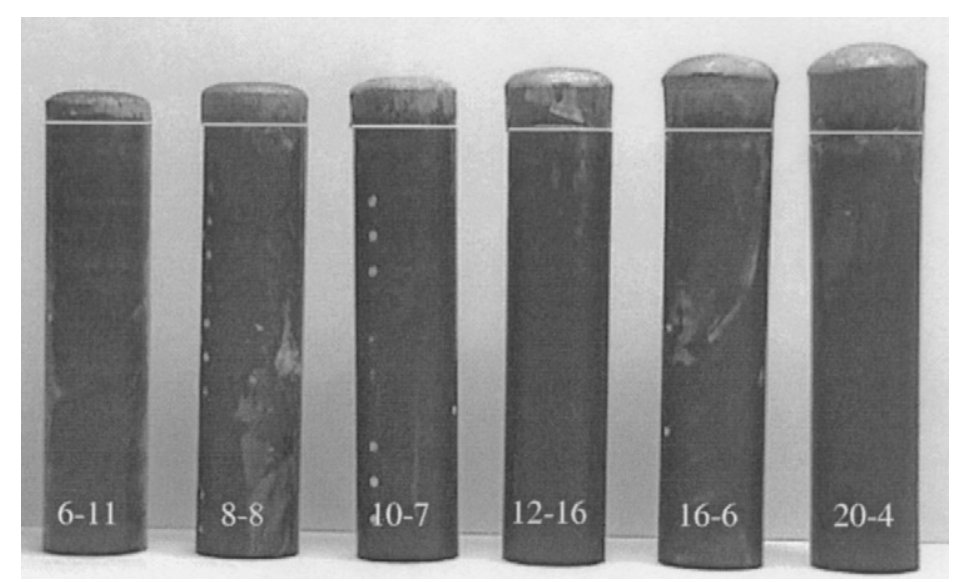

Fig. 14. Projectiles and plugs from tests in targets with varying thickness.

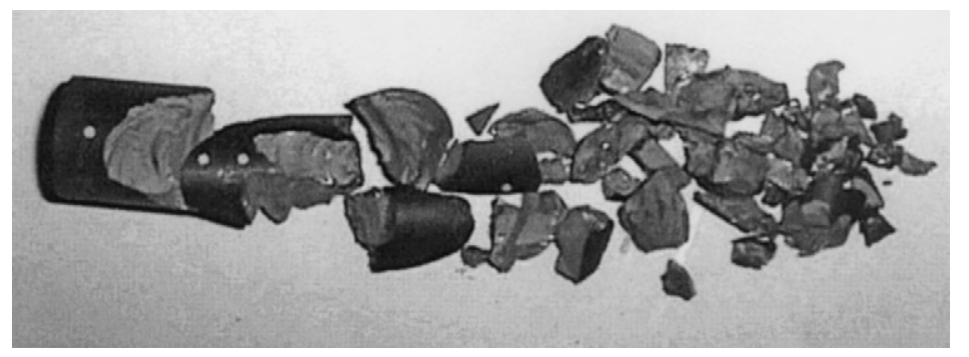

Fig. 15. Shattered projectile after impact of $30 \mathrm{~mm}$ thick target plate (test \# 30-3).

digital high-speed camera images. By computing the centre of gravity based on the intensity of the pixels within each white fiducial mark (see Fig. 12) it is possible to determine the change in velocity with sub-pixel resolution. This method was used to obtain the velocity-time curves presented in Fig. 16, all from tests at an impact velocity near the ballistic limit. The almost linear drop in velocity with time is found to increase both with initial velocity and target thickness. After fracture the curve flattens and shows a constant velocity. Note that the perforation time is nearly constant and about $100 \mu$ s in these tests.

Plug thickness as a function of target thickness and projectile impact velocity is plotted in Fig. 17. As long as the target plate is relatively thin, say $h_{\mathrm{t}} \leqslant 12 \mathrm{~mm}$, the thickness of the plug from impact tests close to the ballistic limit is almost identical to the original plate thickness. Thus, plastic compression and lateral material flow before complete formation of the plug is considered small, and it follows that shear banding is the dominant failure mode. For thicker plates a linear reduction in plug thickness with target thickness is found when $h_{\mathrm{t}} \leqslant 20 \mathrm{~mm}$ (Fig. 17(a)). The plug thinning is also seen to increase with impact velocity, and in particular for the thickest plates (Fig. 17(b)). The diameter of the plug is almost identical to the final diameter of the projectile nose. The increase in projectile nose diameter $\left(\Delta D=1 \times 10^{-10} v_{\mathrm{i}}^{4.0238}\right)$ from all tests is plotted in Fig. 18 as a function of impact velocity. As seen, the deformation to fracture is well predicted by a power law function. A similar behaviour was found for the reduction in projectile length $(\Delta L=$ 


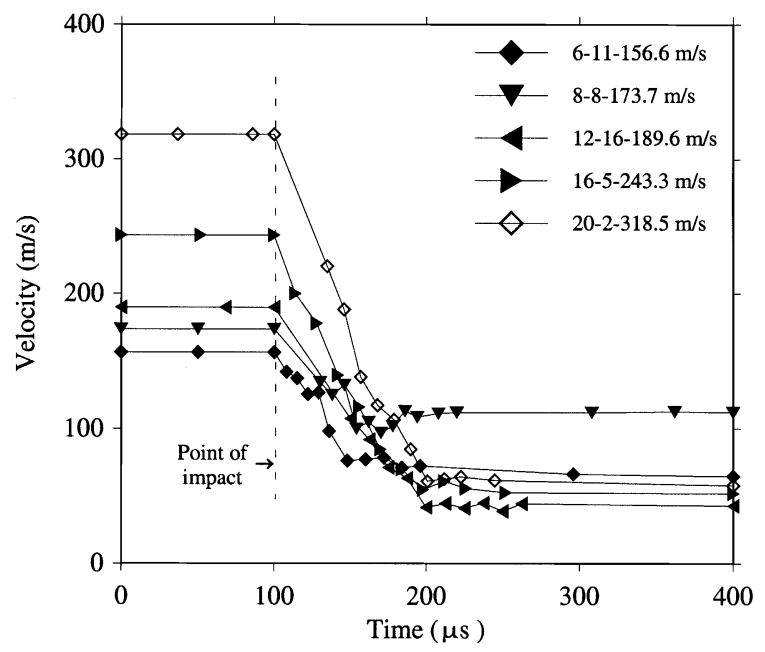

Fig. 16. Velocity-time curves measured from high-speed camera images.
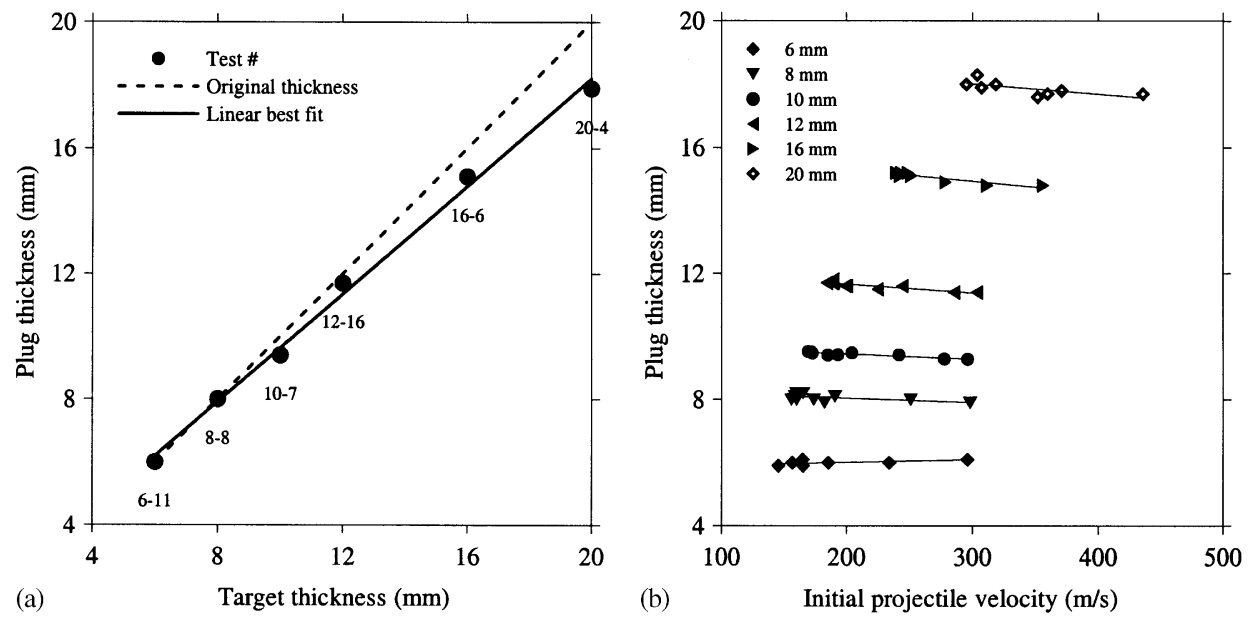

Fig. 17. Plug thickness as function of (a) target thickness and (b) projectile impact velocity.

$\left.1 \times 10^{-10} v_{i}^{4.0583}\right)$. These observations validate the assumption that projectile deformations take place at impact, and are more affected by impact velocity than by target thickness. Accordingly, when the impact velocity gets high the amount of energy absorbed in projectile plastic deformation becomes considerable. Several more details from the experimental tests are given in Table 2.

\section{Empirical equations}

In practical engineering or design, it may be difficult or even impossible to obtain detailed information on a specific penetration problem in a short term. Therefore, in order to have a 


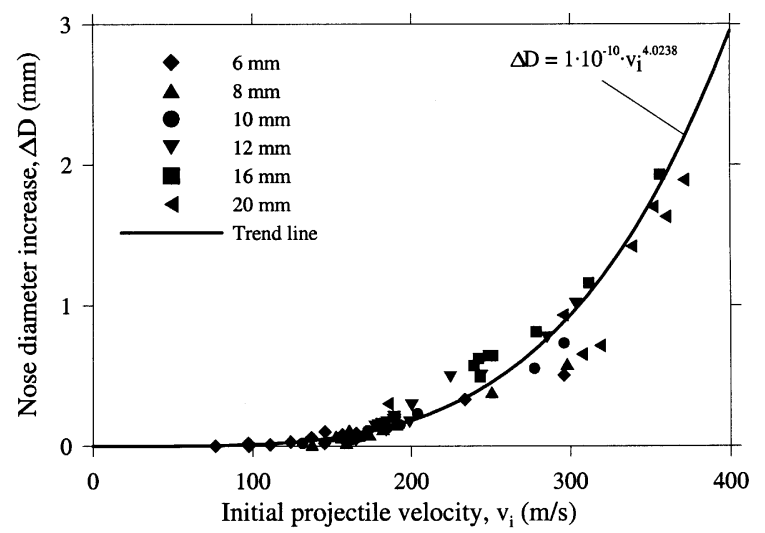

Fig. 18. Projectile nose diameter increase $(\Delta D)$ versus initial projectile velocity $\left(v_{\mathrm{i}}\right)$.

preliminary prediction of the structural response for a given load, an empirical approach is often used. However, the method may give considerable errors and it is both difficult and hazardous to extrapolate empirical equations outside their often strictly prescribed limits. Also, the empirical approach gives little if any insight into the dynamic structural response or the underlying mechanisms behind the penetration process. Keep in mind that most empirical models are based on least-square curve fits to a limited number of experimental data, often by means of dimensional analysis.

Typical and well-known empirical models frequently found in the literature for the normal impact of steel plates by blunt cylindrical projectiles have been quoted and discussed in [30]. Results from calculations using some of these formulas are shown in Fig. 19, while their stated limitations are as given in Table 4. It is important to notice that several of the plotted models do not completely satisfy the experimental limitations in this study. Model results were obtained using typical input data from the penetration tests, where hardened blunt projectiles $\left(D_{\mathrm{p}}=20 \mathrm{~mm}, \quad L_{\mathrm{p}}=80 \mathrm{~mm}, \quad m_{\mathrm{p}}=197 \mathrm{~g}\right)$ impacted circular Weldox $460 \mathrm{E}$ steel plates $\left(d_{\mathrm{t}}=500 \mathrm{~mm}, \sigma_{\mathrm{y}}=490 \mathrm{MPa}, \sigma_{\mathrm{u}} / \sigma_{\mathrm{y}}=1.2\right)$ of varying thickness in the sub-ordnance velocity regime. Fig. 19 illustrates the severe dispersion in predicted results. For the thinnest plate used in the calculations, the difference in critical velocity between the Recht-Ipson and the SRI model is $340 \%$. The Recht-Ipson model is in fact not an empirical model, since no empirical constants are needed. However, it has been derived from dimensional analysis and is therefore presented in this context. The scatter is also significant for the thickest plate, where the deviation in ballistic limit between the AEA and THOR equations is $260 \%$. It is seen that the Recht-Ipson model provides the closest fit to the experimental data. This is also the only model that gives a conservative estimate of the target response. However, also the AEA equation is relatively close, while both the BRL and the modified De Marre formulas give reasonable agreement with the experimental results. Nevertheless, it seems clear that special attention must be taken if the empirical approach is to be used in design. For that reason, both the analytical and numerical approach are of greater interest in structural impact, since these more physically based methods lend themselves better to applications over a wider range of problems. 


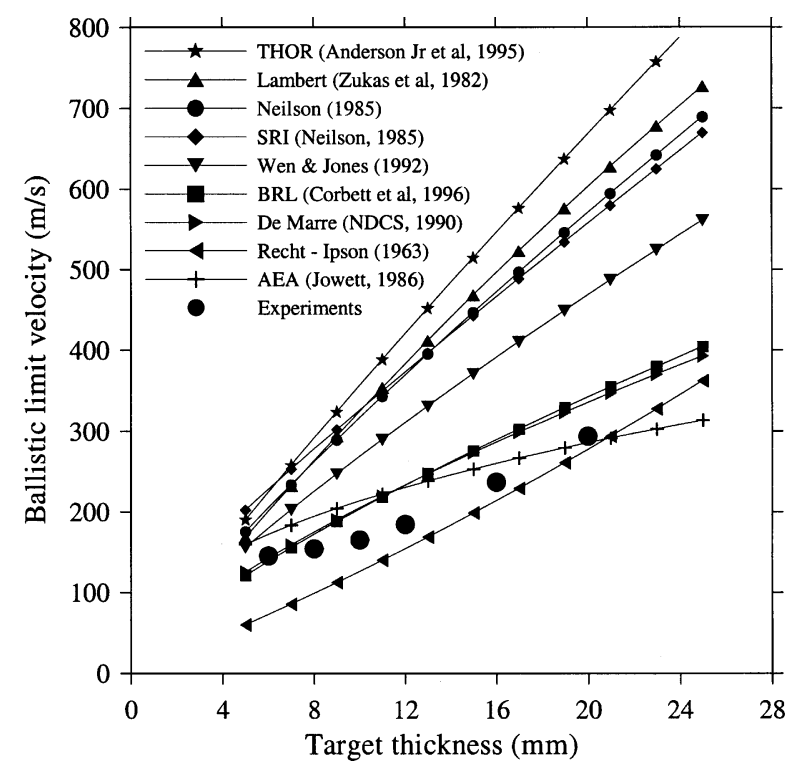

Fig. 19. Comparison between data from empirical models and experimental results.

Table 4

Stated limits for experiments and empirical models [30]

\begin{tabular}{llllllll}
\hline & Limits & \multicolumn{7}{l}{} \\
\cline { 2 - 7 } & $h_{\mathrm{t} / D_{\mathrm{p}}}$ & $h_{\mathrm{t}} / L_{\mathrm{p}}$ & $L_{\mathrm{p}} / D_{\mathrm{p}}$ & $d_{\mathrm{t}} / D_{\mathrm{p}}$ & $d_{\mathrm{t}} / h_{\mathrm{t}}$ & $\sigma_{\mathrm{u}}(\mathrm{MPa})$ & $v_{\mathrm{i}}(\mathrm{m} / \mathrm{s})$ \\
\hline Experiments & $0.3-1.5$ & $0.08-0.38$ & 4 & 25 & $17-83$ & 580 & $100-500$ \\
THOR & - & - & -3 & - & - & - & - \\
De Marre & - & - & - & - & - & - & $200-900$ \\
SRI & $0.1-0.6$ & $0.002-0.05$ & $10-50$ & $5-8$ & $8-100$ & - & $21-122$ \\
BRL & - & - & - & - & - & - & - \\
Neilson & $0.14-0.64$ & - & $>13$ & $4-22$ & - & - & - \\
AEA & $0.1-0.65$ & - & $2-8$ & $>12$ & - & $315-483$ & $40-200$ \\
Wen and Jones & $0.4-1.6$ & - & - & 40 & $25-100$ & $340-440$ & $<20$ \\
Recht and Ipson & $<0.5$ & $<0.5$ & - & - & - & - & - \\
Lambert [See [30] for database] & & & & & & \\
\hline
\end{tabular}

\section{Analytical models}

Analytical models can range anywhere in complexity from simplified one-dimensional equations based on a single physical mechanism to very advanced two- or three-dimensional models derived from combined local mechanisms, assumed global deformation fields and different failure criteria [4,5]. Many models have been proposed in the literature, and a thorough review on 
Table 5

Input to the Wen-Jones [31] model

Target material properties (Weldox $460 \mathrm{E}$ ) and projectile constants

\begin{tabular}{llllllllll}
\hline$\sigma_{\mathrm{y}}(\mathrm{MPa})$ & $\sigma_{\mathrm{u}}(\mathrm{MPa})$ & $D\left(\mathrm{~s}^{-1}\right)$ & $q$ & $\tau_{\mathrm{u}}(\mathrm{MPa})$ & $\gamma_{\mathrm{u}}$ & $n$ & $G(\mathrm{~kg})$ & $d(\mathrm{~m})$ & $R(\mathrm{~m})$ \\
\hline 490 & 580 & $4.6 \times 10^{7}$ & 7.33 & 570 & 1.4 & 0.12 & 0.197 & 0.020 & 0.25 \\
\hline
\end{tabular}

recent analytical models in the sub-ordnance impact velocity regime can be found in [3]. In contrast, only a few comparisons between model results and experimental data exist. Here, three well-known analytical models developed to calculate the ballistic response of metal plates impacted by blunt projectiles are briefly presented. The models are then used to predict ballistic limit velocities and curves for the problem investigated experimentally. It is important at this stage to emphasise that other models may give better agreement with the experimental results than those chosen in this particular study.

\subsection{The Wen-Jones [31] model}

Wen and Jones [31] proposed an approximate quasi-static model for the behaviour of punchimpact-loaded metal plates. Based on the principle of virtual work, load-deflection relationships were first obtained and then used to predict the energy absorbing capacity of plates subjected to low-velocity impact causing perforation. Many approximations and simplifications were introduced into the analysis. However, the theoretical predictions on fully clamped steel plates were found to be in good agreement with experimental results from $[22,7,32,6]$ when material strain rate effects were taken into account. It was further provided that perforation did not occur by adiabatic shear instability. The main equations from the model are given in Appendix A, while details in the theoretical description can be found in the original paper. Note also that a contemporaneous analysis was proposed by [33], which gives additional light on the aspects of this type of analytical modelling.

For this particular problem, necessary input data to the model is given in Table 5. The engineering stress-strain curve from quasi-static tensile tests was used to determine the yield stress $\sigma_{\mathrm{y}}$ and the ultimate stress $\sigma_{\mathrm{u}}$ (Fig. 5 and Table 1). The Cowper-Symond coefficients $D$ and $q$ in Eq. (A.7) were fitted to the experimental data from tensile tests on smooth specimens from the target material at different strain rates [12]. As seen, the calibrated values of $D$ and $q$ are quite different from those given in [34] for mild steel. Material constants for the local critical shear strain $\gamma_{\mathrm{u}}$, the work-hardening index $n$ and the critical shear stress $\tau_{\mathrm{u}}$ are determined in two different ways. First, the method described in the Wen-Jones paper was used. Here, $\gamma_{u}$ and $n$ were taken to be the "typical" values of 0.8 and 0.25 for mild steel, respectively, and $\tau_{\mathfrak{u}}$ was allowed to vary with target thickness as proposed by Langseth and Larsen [7]. The behaviour of a $12 \mathrm{~mm}$ thick Weldox $460 \mathrm{E}$ steel plate using these coefficients is plotted as the curve "Calibration I" in Fig. 20. However, this is a rather inaccurate material description if compared to the material tests. Therefore, in the second approach, $\gamma_{\mathrm{u}}, n$ and $\tau_{\mathrm{u}}$ were fitted to a shear stress-strain curve generated from the viscoplastic-damage model proposed by Børvik et al. [20]. This model was calibrated for Weldox $460 \mathrm{E}$ steel and the shear stress is plotted for a strain rate of $10^{4} \mathrm{~s}^{-1}$ in Fig. 20. The 


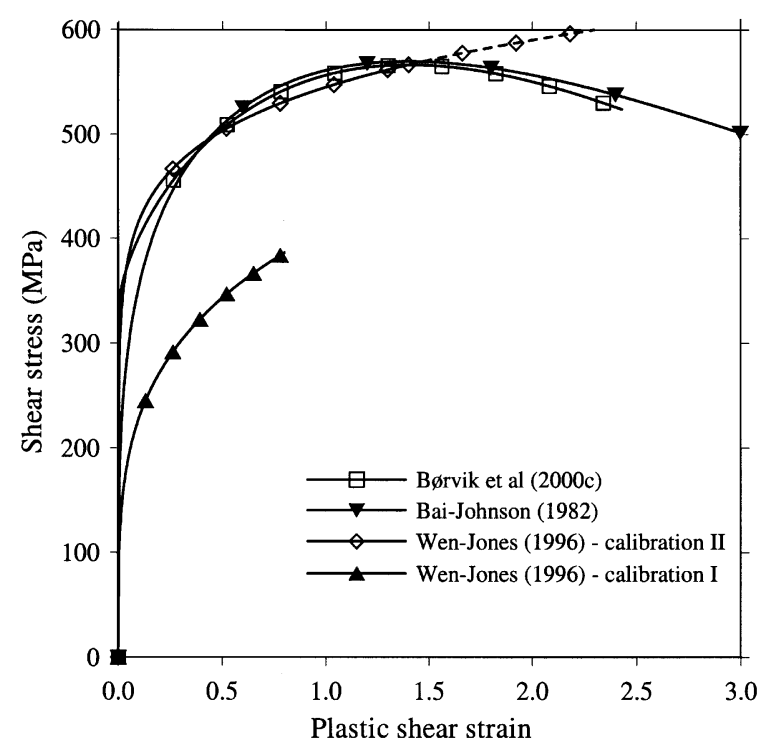

Fig. 20. Material behaviour from different constitutive relations.

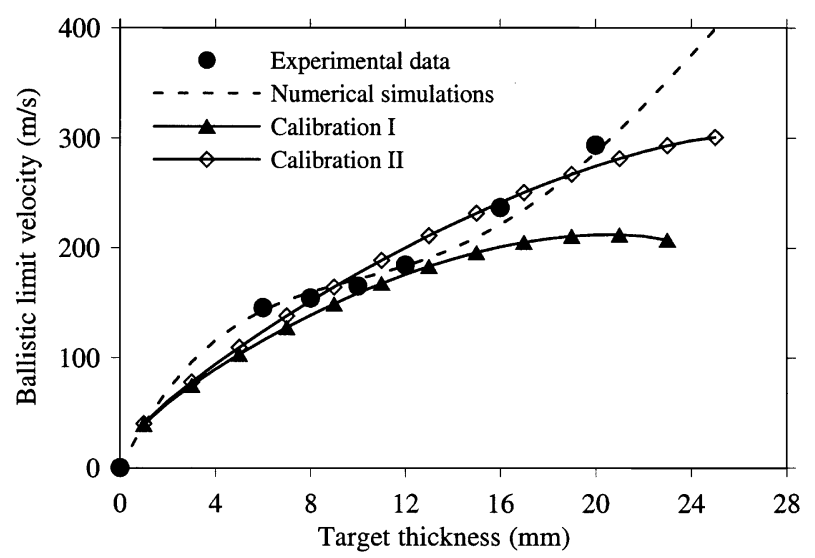

Fig. 21. Ballistic limit velocity versus target thickness obtained from the Wen-Jones [31] model. The broken line is based on a best fit to the numerical results in Section 6.

assumed strain rate of $10^{4} \mathrm{~s}^{-1}$ ensures adiabatic conditions according to Lindholm and Johnson [35], and this is also a reasonable value for the strain rate in the shear zone although local values may be much higher. A best fit to the viscoplastic-damage model gave the material constants for $\gamma_{\mathrm{u}}, n$ and $\tau_{\mathrm{u}}$ in Table 5. The material behaviour using these values is shown as the curve "Calibration II" in Fig. 20. It is seen that the latter gives a good description of the local material behaviour during penetration, even though material softening is not included.

Ballistic limit velocities calculated from Eq. (A.8) with input data as given above and in Table 5 are plotted versus target thickness in Fig. 21. The broken line in Fig. 21 is based on a best fit to the numerical results presented in Section 6. Reasonably agreement is obtained between the model and the experimentally obtained ballistic limits for plate thicknesses up to $16 \mathrm{~mm}$ if the material 
coefficients are fitted to the "Calibration II"-curve in Fig. 20. For thicker plates, the deviation is more distinct. It is seen that the Wen-Jones model does not describe the kink in the response curve (Fig. 9) with target thickness. Note also that when the static collapse load $F_{\mathrm{c}}$ exceeds the critical value of the transverse load $F_{\mathrm{u}}$ in Eq. (A.7), the critical transverse displacement of the plate becomes negative and the Wen-Jones model breaks down. Here, this occurs at a target thickness of about $26 \mathrm{~mm}$. Furthermore, the model does not give a separate expression for the residual versus impact velocity curve after plugging. However, residual projectile velocities can be estimated using the Recht-Ipson model [25] in Eq. (1) with $a=m_{\mathrm{p}} /\left(m_{\mathrm{p}}+m_{\mathrm{pl}}\right), m_{\mathrm{pl}}=\rho \pi r_{\mathrm{p}}^{2} h_{\mathrm{t}}$ and $\mathrm{p}=2$. This model gives good agreement with the experimental data as long as the projectile deformation is small.

Finally, the Wen-Jones model gives an interesting relation between projectile indentation and shear failure. It is shown in Eq. (A.3) that if the critical shear strain $\gamma_{u}$ is equal to the fitted value of 1.4 , the critical depth of indentation is about $h_{\mathrm{t}} / 2$, i.e. plugging occurs when the projectile has penetrated half the target thickness. This value is used in simplified models as a failure criterion and has been observed both experimentally and numerically (e.g. [12]). Here, the extension of the shear zone $e$ is assumed to increase linearly with target thickness. Calculated relative maximum target deformations $\left(W_{\mathrm{os}}\right)$ from Eq. (A.7) versus target thickness are compared with both experimental and numerical values in Fig. 10. The Wen-Jones model seems to give an excellent description of the global target deformation during impact. The model was also used to estimate the average value of the strain rate (see $\dot{\varepsilon}_{\mathrm{m}}$ in Eq. (A.7)). The strain rate was calculated to lie between 1 and $100 \mathrm{~s}^{-1}$. However, this is with the exception of the shear zone, where the strain rate is assumed to be much higher.

\subsection{The Bai-Johnson [36] model}

Bai and Johnson [36] developed a mechanical model for plugging of metal plates based on adiabatic shear instability. The model consists of three basic elements; the kinetic energy equation connecting the projectile and plug, the constitutive equation and the relationship between the displacement of the plug and the shear strain. Here, only a short summary of the most important features of the model is given in Appendix B. It is referred to the paper by Bai and Johnson [36] for a more detailed description of the model.

By assuming the von Mises yield criterion and the normality rule, the material constants required in Eq. (B.5) were determined in a similar way as for the Wen-Jones model using the calibrated viscoplastic-damage model from [20] for an assumed strain rate of $10^{4} \mathrm{~s}^{-1}$. A plot of the adiabatic shear stress-strain curve derived from Eq. (B.5) is shown in Fig. 20, while the constants from the fit are given in Table 6 together with other necessary input data to the model.

Table 6

Input to the Bai-Johnson [36] model

Target material properties (Weldox $460 \mathrm{E}$ ) and projectile constants

\begin{tabular}{llllllll}
\hline$\tau_{\mathrm{u}}(\mathrm{MPa})$ & $\gamma_{\mathrm{u}}$ & $n$ & $\rho\left(\mathrm{kg} / \mathrm{m}^{3}\right)$ & $C_{\mathrm{v}}(\mathrm{J} / \mathrm{kgK})$ & $\theta_{0}(\mathrm{~K})$ & $M(\mathrm{~kg})$ & $a(\mathrm{~m})$ \\
\hline 570 & 1.4 & 0.25 & 7850 & 452 & 293 & 0.197 & 0.010 \\
\hline
\end{tabular}




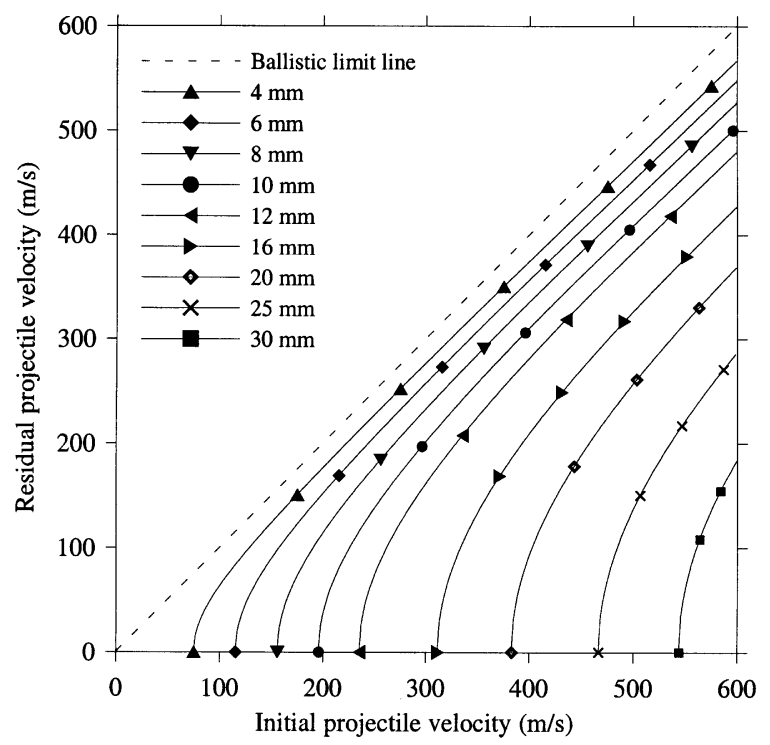

Fig. 22. Residual versus impact velocity curves obtained from the Bai-Johnson [36] model.

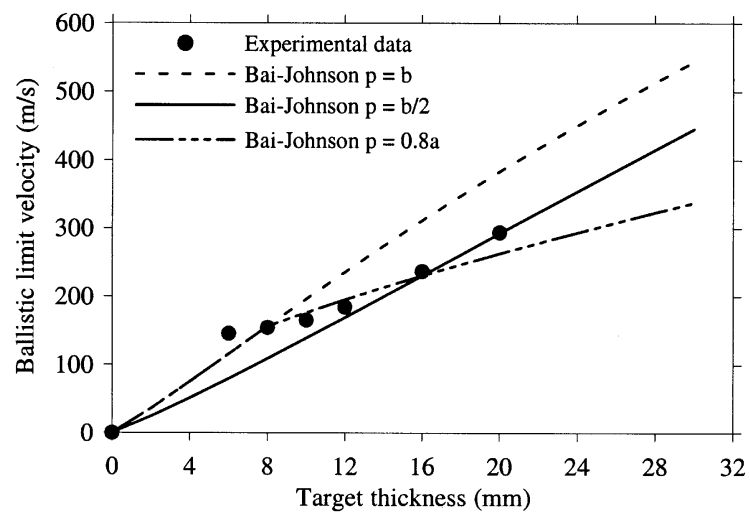

Fig. 23. Ballistic limit velocity versus target thickness obtained from the Bai-Johnson [36] model.

Furthermore, Bai and Johnson used a very simple failure criterion. They assumed that the penetration process terminates when the penetration depth $p$ equals the target thickness $h_{\mathrm{t}}=b$. However, microscopic examinations of impacted targets have indicated that complete separation of the plug often occurs at a penetration depth $p$ between $b / 2$ and $b$ [37]. Residual versus impact velocity curves as obtained from Eq. (B.2) for $p=b$ are shown in Fig. 22, while ballistic limit velocities calculated from Eq. (B.4) versus target thickness for $p=b / 2$ and $b$ are plotted and compared to the experimental data in Fig. 23. It is seen from Fig. 23 that an upper limit for the ballistic resistance of the target is obtained when $p=b$, while good agreement with the experimental data is found for $b \geqslant 12 \mathrm{~mm}$ when $p=b / 2$. The latter corresponds well with the prescribed validity range of the Bai-Johnson model. Another possibility is to include a new simple 
fracture criterion in the model. From Eq. (B.7) it is found that

$$
p_{\mathrm{u}}=\frac{n}{1-n} a \gamma_{\mathrm{f}} \leqslant b
$$

which for Weldox $460 \mathrm{E}$, where $\gamma_{\mathrm{f}} \approx 2.4$ for $\dot{\varepsilon}=10^{4} \mathrm{~s}^{-1}$ and $n=0.25$ (Table 6), leads to $p_{\mathrm{u}}=0.8 a$. Thus, the penetration depth to failure is independent of target thickness. The obtained ballistic limit velocities using this criterion is also plotted versus target thickness in Fig. 23, and the results agree reasonably well with the experimental data. For the thinnest plates the fracture mode is not adiabatic shear instability, but rather a combined deformation mode of bending, shear and tension. As seen both in Figs. 11 and 12, global target deformation is a prominent energy absorbing mode for plates thinner than $12 \mathrm{~mm}$. This mode is not accounted for in the BaiJohnson model, and the ballistic resistance for the thinnest plates is therefore underpredicted. The shape of the residual versus impact velocity curves in Fig. 22 are in good agreement with the experimental results, considering that projectile deformation which is considerable for the thickest plates (see Fig. 18) is not accounted for in the model.

It is also possible to extract detailed information such as shear strain, penetration force, energy absorption in the shear zone, temperature increase, projectile-plug velocity and shear strain rate as a function of penetration depth $p$ from the Bai-Johnson model. The model was therefore utilised to estimate details associated with the shear plugging in a microscopic study on partially perforated target plates [24], giving reasonable agreement with the experimental observations. The duration of the penetration process as defined by Eq. (B.11), i.e. the time until a through-targetcrack has occurred so that the shear zone can absorb no more energy, is also of great interest. If $p=b / 2$, it is found that the perforation time is almost constant and about $80 \mu$ sindependent of target thickness. Perforation times from the Bai-Johnson model are compared with experimental and numerical results in Fig. 27.

\subsection{The Ravid-Bodner [38] model}

Ravid and Bodner [38] proposed an advanced two-dimensional analytical model of the penetration and perforation process for intermediate thick plates struck normally by rigid projectiles, where the analysis parallels procedures used in metal forming operations. Due to the mathematical complexity of the model, no equations are quoted herein and it is referred to the original paper for further details. In the model, elastic effects are neglected, the target material is assumed rigid-viscoplastic and an overall work-rate balance of the rigid projectile/target system is established. The position and motion of the target material are determined at all times during the perforation process, and no empirical information is required. Global target deformation, shock compression, projectile deformation and erosion effects are not included in this model, but are treated in other works by the authors. The analysis is thus applicable for normal impact of rigid projectiles into ductile targets at velocities from about 200 to $1000 \mathrm{~m} / \mathrm{s}$. Target thicknesses could range from fairly thin, i.e. on the order of the projectile diameter, to very thick (Bodner and Ravid [39]). An essential feature of the analysis is the use of postulated, physically motivated deformation mechanisms in conjunction with the upper bound theorem of plasticity that is modified to include dynamic effects. A plastic flow velocity field is defined in the target that is consistent with the boundary conditions, the geometrical constraints, and continuity and mass 
conservation requirements. Five successive and interconnected stages of the penetration process, each represented by a distinct plastic flow field, are computed. Various failure modes are considered throughout the different stages, and a number of plugging modes can occur due to a limiting strain criterion or by adiabatic shear band formation. The available failure modes have been classified as adiabatic shearing, brittle failure in shear, plug formation and ductile failure. The model is self-contained and capable of predicting the exit velocities of the projectile and plug. It also determines the bulge and plug shape, provides the velocity-time and force-time histories of the process, and describes a number of geometrical features during the different deformation states of the target plate. The original model was developed for blunt projectiles, but other nose shapes that required changes in the plastic flow fields were considered in a paper by Ravid et al. [40]. This paper also discussed the plastic flow field due to deep penetration and thermal softening.

Due to the mathematical complexity of the model, it requires numerical treatment. Here, the analyses were carried out using the computer program SHAY-1S from [41]. In the program, only standard input such as geometrical data of the projectile and target and conventional physical and mechanical properties are requested. The model output is arranged in sorted data files, giving typical terminal ballistic data, geometrical data, and scientific values such as strain rate, work rate, etc. Thus, the amount of detailed information provided by the model is extensive, and this may be used for validation purposes. In the analyses, the target material is assumed to be rigid-plastic with a flow stress defined as a function of strain rate. Ravid and Bodner suggested that work hardening should be considered by taking the flow stress $\sigma_{0}$ as the average value of plastic work over the full strain range. Here, a somewhat simpler approach is adopted and $\sigma_{0}$ is calculated as

$$
\sigma_{0}=\left(\sigma_{\mathrm{y}}+\sigma_{\mathrm{u}}\right) / 2
$$

where $\sigma_{\mathrm{y}}$ and $\sigma_{\mathrm{u}}$ are the yield and engineering ultimate stress, respectively, at a given strain rate. The strain rate dependence of the flow stress is given by the expression

$$
\sigma_{\mathrm{d}}=\sigma_{0}\left[1+C \log _{10} \dot{\varepsilon}_{\mathrm{eff}}\right]
$$

where $\sigma_{\mathrm{d}}$ is the dynamic flow stress, $\sigma_{0}$ is the quasi-static flow stress, $C$ is a material constant and $\dot{\varepsilon}_{\text {eff }}=\dot{\varepsilon} / \dot{\varepsilon}_{0}$ where $\dot{\varepsilon}$ is the actual strain rate and $\dot{\varepsilon}_{0}=5 \times 10^{-4} \mathrm{~s}^{-1}$ is taken as the quasi-static strain rate. Values of $C$ have been fitted to the experimental data from tensile tests at elevated rates of strain [20], both when $\sigma_{0}=\sigma_{\mathrm{y}}$ and when $\sigma_{0}$ is as given by Eq. (3). The former was used in this study because it gives a conservative estimate of the flow stress. Based on earlier observations using blunt projectiles [20], frictional effects are neglected, i.e. $\mu_{\mathrm{f}}=\mu_{1}=\mu_{\mathrm{p}}=0$. Based on von Mises the model assumes that failure occurs when $\gamma=\gamma_{\mathrm{f}}$, where $\gamma_{\mathrm{f}}=\sqrt{3} \varepsilon_{\mathrm{f}}$ and $\varepsilon_{\mathrm{f}}$ is the true (logarithmic) strain to failure measured from the maximum reduction of cross-section area in a standard tensile test. This is a simplification to the failure process during shear plugging. Both Table 1 and Fig. 5 indicate that Weldox $460 \mathrm{E}$ is a very ductile material. The true strain to failure measured in a quasi-static tensile test is about $160 \%$, which is reduced to $120 \%$ at a strain rate of $10^{4} \mathrm{~s}^{-1}$ [12]. However, the Ravid-Bodner model was developed for moderately ductile materials such as armour steel. A condition such as the Considère's criterion for diffuse necking at maximum load [42], i.e. where the rate of strain hardening equals the stress, was therefore used. For Weldox $460 \mathrm{E}$ this criterion is realised at a true strain $\varepsilon_{\mathrm{u}}$ of about $20 \%$ (see Fig. 5). The variation of $\varepsilon_{\mathrm{u}}$ with strain rate has earlier been found to be small [20]. Thus, the model assumes a constant shear stress until fracture at a constant failure strain. If compared to Fig. 20, the material 
Table 7

Input to the Ravid-Bodner [38] model

Target material properties (Weldox $460 \mathrm{E}$ ) and projectile constants

\begin{tabular}{lllllllll}
\hline$\sigma_{\mathrm{y}}(\mathrm{MPa})$ & $\sigma_{0}(\mathrm{MPa})$ & $C_{\mathrm{y}}$ & $C_{0}$ & $\mu$ & $L_{\mathrm{p}}(\mathrm{mm})$ & $D_{\mathrm{p}}(\mathrm{mm})$ & $\rho\left(\mathrm{kg} / \mathrm{m}^{3}\right)$ & $\varepsilon_{\mathrm{u}}$ \\
\hline 490 & 550 & 0.0337 & 0.0254 & 0.0 & 80 & 20 & 7850 & 0.20 \\
\hline
\end{tabular}

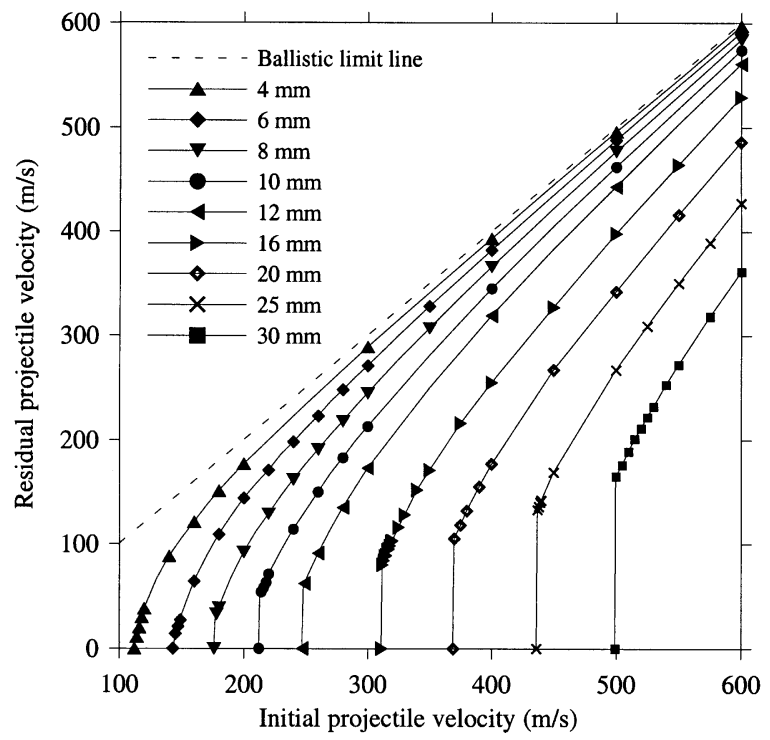

Fig. 24. Residual versus impact velocity curves obtained from the Ravid-Bodner [38] model.

behaviour using this model is quite different from the other models. Even though the model is selfcontained, indicating that there is no demand for empirical constants, one empirical input parameter regarding the fracture mode is needed. Here, the almost cylindrical plug as shown in Fig. 14 is close to the P2 condition described in the paper by Ravid and Bodner [38], and is therefore used in all calculations. Also the adiabatic shearing (AS) option was tested, but the P2 condition provided in general better results. The AS option, which leads to a discontinuity in velocity between the different surfaces, is recommended when adiabatic shear bands are expected in the target. That adiabatic shear bands may appear in the target plates during the penetration process was revealed in a micro-mechanical study by Børvik et al. [24]. Necessary input data to the Ravid-Bodner model is given in Table 7.

Calculated residual versus impact velocity curves are given in Fig. 24. The computational step in all calculations was chosen equal to $0.1 \mathrm{~mm}$, since the solution was found too coarse using the recommended value of $1 \mathrm{~mm}$. In contrast to the experimental results, the "jump" in residual velocity at the ballistic limit seems to increase with target thickness and initial projectile velocity. Since projectile plastic deformation is not accounted for in the model, the ballistic limit curves approaches the ballistic limit line for a target of zero thickness as the velocity is increased. Ballistic limit velocities versus target thickness are plotted in Fig. 25. The discrepancy between model 


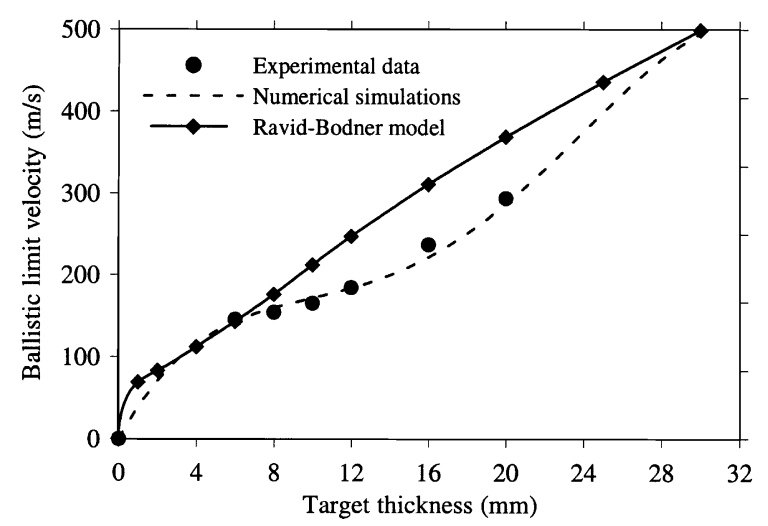

Fig. 25. Ballistic limit velocity versus target thickness obtained from the Ravid-Bodner [38] model. The broken line is based on a best fit to the numerical results in Section 6 .
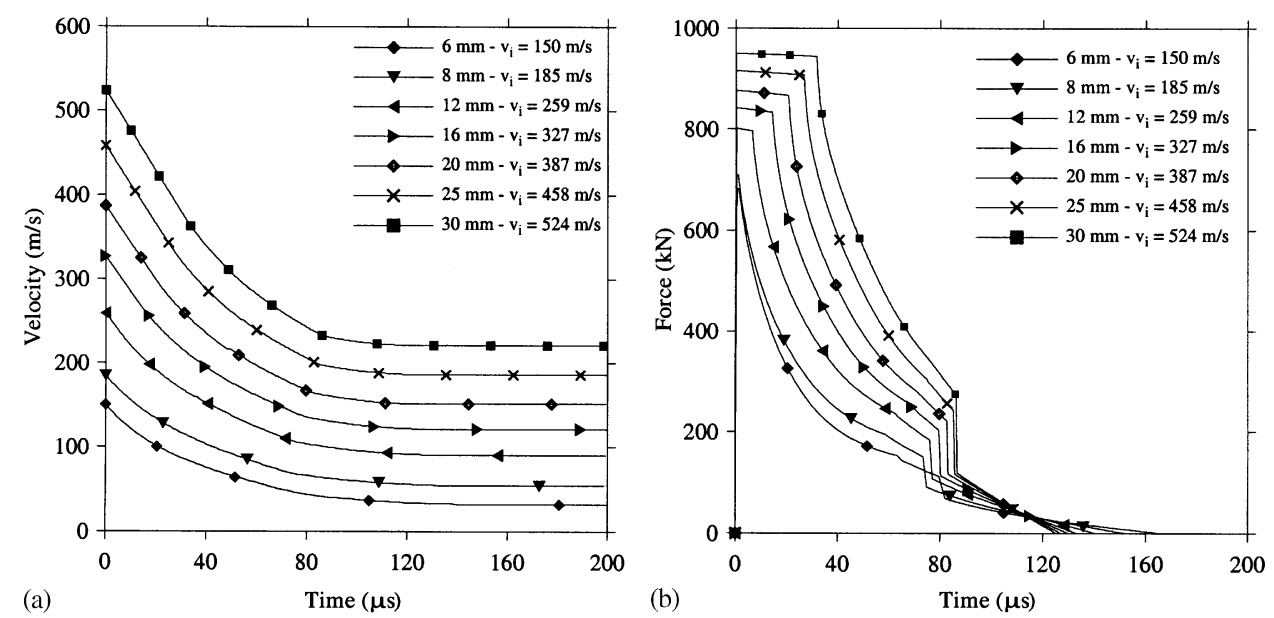

Fig. 26. Predicted projectile (a) velocity and (b) resisting force versus time for targets of different thickness during perforation using the Ravid-Bodner [38] model.

predictions and experimental results are considerable in the intermediate thickness range. At the extreme ends, on the other hand, the model predictions correspond quite well with the plotted trend line based on the numerical simulations. A number of factors may be responsible for the deviation in the results. Thickness or material property variations of the target, non-perfect impacts, shock waves, etc., may all influence the outcome. However, since the applied version of the Ravid-Bodner model does not include global target deformation, it seems reasonable that it fails to describe the kink in the response curve when the global deformation mode changes.

Predicted histories of projectile velocity and resisting force versus penetration time for different target thicknesses are given in Fig. 26. For these curves, the impact velocity of the projectile is approximately 5\% above the respective ballistic limits. In the velocity-time curves in Fig. 26(a), the drop in velocity after impact shows a weak linear increase with target thickness, while the perforation time is almost constant. It appears that the different curves do not intersect, and the 


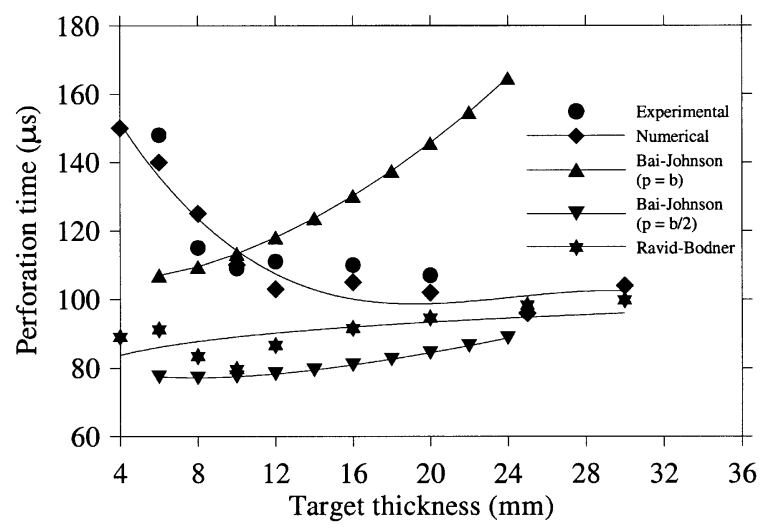

Fig. 27. Perforation times versus target thickness at impact velocities close to the respective ballistic limits.

minimum residual projectile velocity is seen to increase linearly with target thickness. Computed perforation times for different target thicknesses are plotted and compared with the experimental data in Fig. 27. An increase in velocity drop indicates an increase in interface force, as shown in the corresponding force-time curves in Fig. 26(b). The projectile indentation during stage 1 is found to be very short $(t \sim 1 \mu \mathrm{s})$ for the thinnest targets, but increases considerably for the thicker targets. Thus, a plateau of constant force is obtained for the thickest plates during this time period. The resisting force then starts to fall in stage 2 (bulge formation) and stage 3 (bulge advancement) due to target mass movement in front of the projectile. A rapid drop in the resisting force occurs in stage 4 (plug formation and exit) due to separation of the plug from the target plate. This is seen to take place almost at the same time, independent of target thickness. Stage 4 continues until the plug is fully ejected from the target. Here, the resisting force in stage 5 (projectile exit) is zero due to the neglecting of frictional effects.

\section{Numerical simulations}

As indicated in Section 3, both the energy absorbing mechanisms and the failure process itself will change during penetration when the target response changes from typically thin plate global deformation towards thick plate shear localisation. It is hence important to investigate if a numerical approach using finite element simulations is able to describe the structural response when such variations are expected to appear. The validation is carried out by simulating all experimental tests presented in Chapter 3 using LS-DYNA, which is a general-purpose, finite element code for analysis of large deformation dynamic response of structures based on explicit time integration, and is therefore suitable for the type of problem under investigation. No attempts are made herein to describe the many algorithms involved in the code, and it is referred to the manuals of LS-DYNA [18,19] for information regarding the numerical scheme.

\subsection{Finite element models}

In a similar way as for the experimental tests, the only variables in the numerical analyses were the initial projectile velocity and the target thickness. For each simulation, a fixed mesh of 4-node 

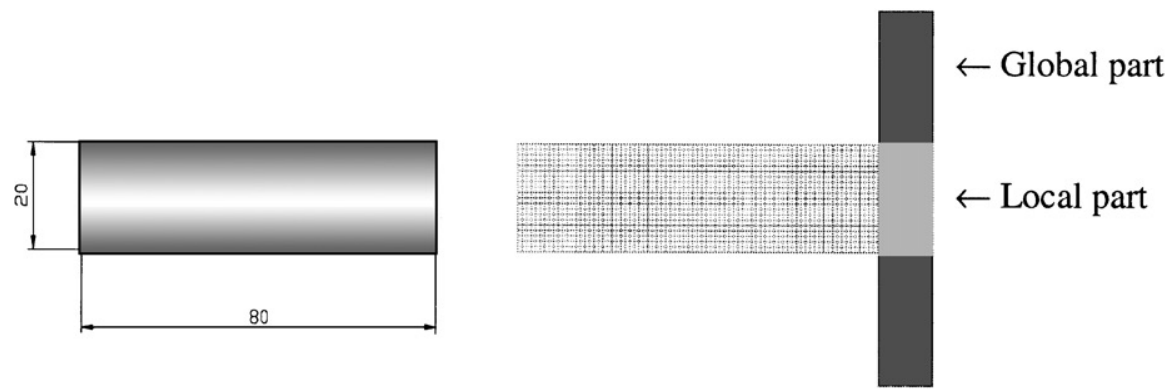

Fig. 28. Geometry of projectile and a plot of the initial FE model showing the local part and a section of the global part of the target.

2D axisymmetric elements with one-point integration and stiffness based hourglass control was used. Fig. 28 gives a plot of the nominal geometry of the projectile and the initial element model for a $12 \mathrm{~mm}$ thick plate, showing a section of the target plate and the blunt projectile just prior to impact. The target was modelled as one local and one global part with identical material properties, see Fig. 28, in order to study global versus local energy absorption during penetration [27]. The boundary between the two parts was placed $0.5 \mathrm{~mm}$ outside the outer radius of the projectile. It should be noted that the target still behaves as one continuous circular plate with a radius of $250 \mathrm{~mm}$ (as in the experiment). The sub-division of the target is a pure numerical technique in order to be able to observe the energy absorption in different parts, and it does not affect the structural response of the target plate during impact. In each run the target plate was fully clamped at the support, while the projectile was given an initial velocity identical to the one measured in the corresponding experiment. The initial size of the smallest element in the impact region was $0.125 \times 0.1 \mathrm{~mm}^{2}$ in all simulations for target thicknesses equal to or less than $12 \mathrm{~mm}$, giving from 40 (4 mm thick targets) to 120 (12 mm thick targets) elements throughout the plate thickness. For thicker targets, the element size in the impact region was increased to $0.25 \times$ $0.2 \mathrm{~mm}^{2}$ in order to reduce the computational time. This gave from 80 (16 mm thick targets) to 150 (30 mm thick targets) elements through the thickness. Contact was modelled using an automatic 2D single surface penalty formulation available in LS-DYNA. In accordance with experimental observations by Børvik et al. [28], frictional effects were neglected for blunt projectiles.

A coupled computational model of viscoplasticity and ductile damage was proposed by Børvik et al. [20] for numerical simulations of the penetration problem. The model is based on work by Johnson and Cook [43,44], Camacho and Ortiz [45], Lemaitre and Chaboche [46] and Lemaitre [47]. It includes linear thermoelasticity, the von Mises yield criterion, the associated flow rule, isotropic strain hardening, strain rate hardening, softening due to adiabatic heating, softening due to isotropic damage evolution and a failure criterion. Model constants have been identified for the target material of Weldox $460 \mathrm{E}$ steel by conducting standard tensile tests on smooth and notched specimens at room temperature, dynamic tests at room temperature and strain rates between $10^{-4}$ and $10^{+3} \mathrm{~s}^{-1}$, and tests on smooth specimens at temperatures between $20^{\circ} \mathrm{C}$ and $500^{\circ} \mathrm{C}$ [20]. The calibrated constitutive model was used to generate the stress-strain curves shown in Fig. 29 for different rates of strain at constant hydrostatic tension $\left(\sigma^{*}=\sigma_{\mathrm{m}} / \sigma_{\mathrm{eq}}=1 / 3\right.$, where $\sigma_{\mathrm{m}}$ is the mean stress). The equation for the equivalent von Mises stress $\sigma_{\mathrm{eq}}$ is also given in Fig. 29. Here, the first 


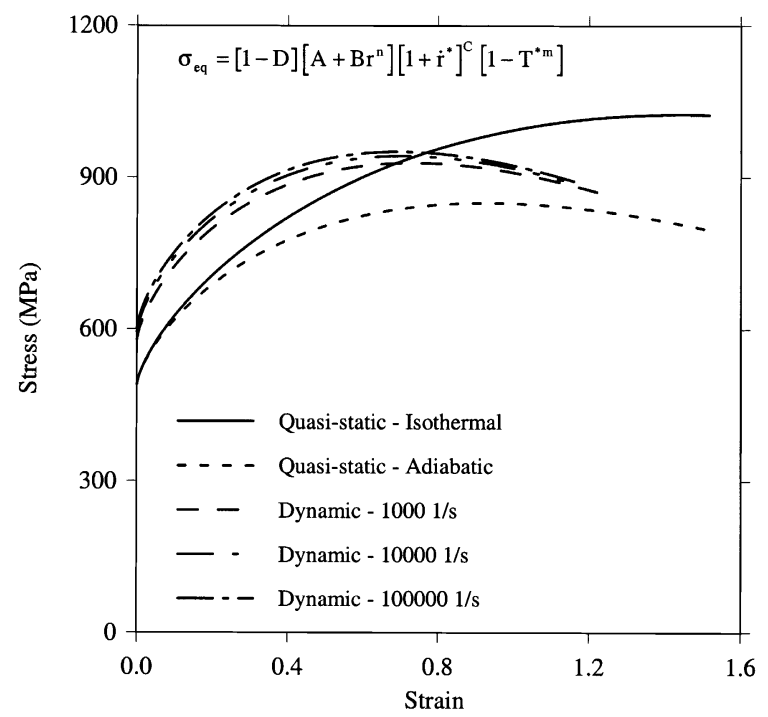

Fig. 29. Calculated quasi-static and dynamic true stress-strain curves for Weldox 460 E steel based on the calibrated viscoplastic-damage model developed by Børvik et al. [20].

bracket describes material degradation due to damage, the second gives the strain hardening, the third defines the effect of strain rate, while the last bracket gives the effect of temperature softening on $\sigma_{\text {eq }}$. To allow crack growth during penetration, the model is coupled with an elementkill algorithm in LS-DYNA that removes damaged elements from the mesh when the damage variable reaches its critical value. The projectile was simply modelled as a bilinear elastic-plastic von Mises material with isotropic hardening. Material constants for the target and projectile are listed in [20]. These constitutive relations enter into the numerical model in all simulations.

Earlier simulations have indicated that the actual problem involving shear localisation and plugging for blunt projectiles is mesh size sensitive [27,20]. To check this, the ballistic limit velocities for several target thicknesses were determined when the number of elements over the thickness varied. Due to a better description of the strain distribution in the localised zone using small elements, it is expected that the ballistic limit velocity will drop with element size. However, due to the viscoplastic material model, the numerical results seem to converge towards a limit solution when the number of elements over the target thickness is sufficiently large, see Fig. 30(a). Thus, the mesh size dependency does not seem to be pathological, and the solution stabilises when the element size becomes small enough. Note also that the numerical results change from nonconservative to conservative estimates of the ballistic limit when the number of elements over the target thickness increases. As seen in Fig. 30(b), the mesh sensitivity is most distinct close to the ballistic limit. At higher velocities, the results are far less affected by mesh size. The mesh sensitivity could be expected since the width of the adiabatic shear band is typically in the order of $10^{1}-10^{2} \mu \mathrm{m}[48,24]$. Here, the CPU-time of the simulations varied from $10 \mathrm{~min}(8 \mathrm{~mm}$ thick target; 20 elements through thickness; initial velocity of $600 \mathrm{~m} / \mathrm{s})$ to approximately $50 \mathrm{~h}(16 \mathrm{~mm}$ thick target; 160 elements through thickness; initial velocity of $230 \mathrm{~m} / \mathrm{s}$ ) on a HP C360 workstation. In order to reduce the computational time, which is affected both by the element size and number, the element size was somewhat enlarged towards the clamp. A further reduction of 

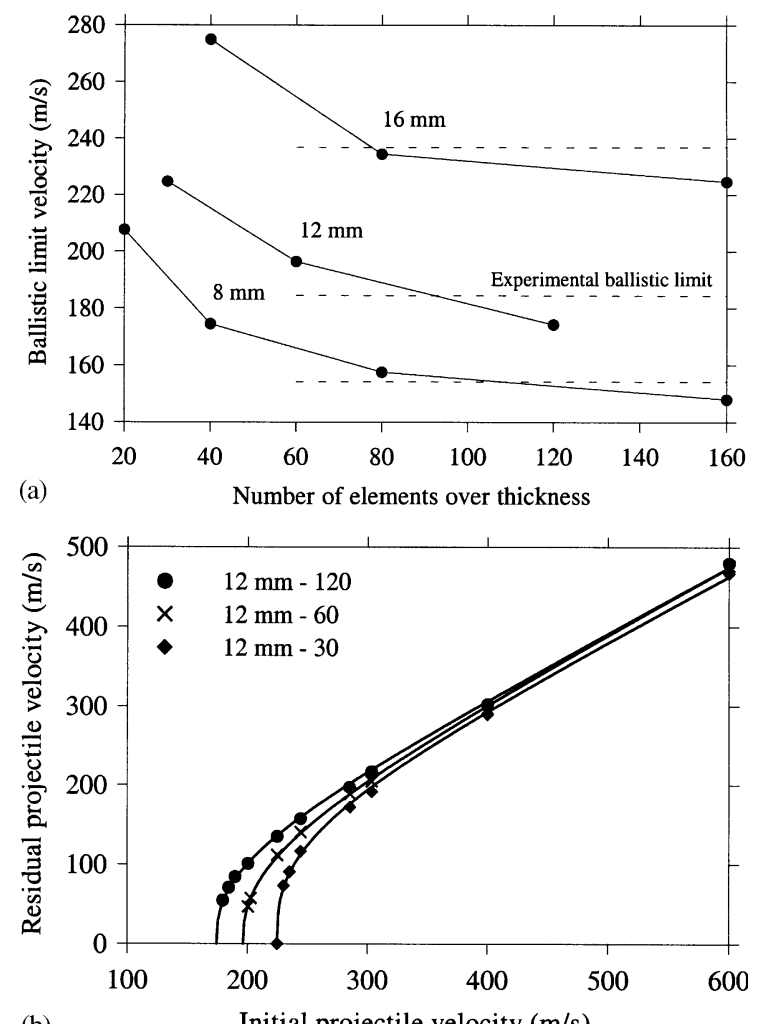

(b)

Initial projectile velocity $(\mathrm{m} / \mathrm{s})$

Fig. 30. Mesh sensitivity of numerical simulations.

the element size in the impact region is for the time being restricted due to limitations in the computational resources.

\subsection{Numerical results}

Residual versus impact velocity curves based on the numerical results are constructed in Fig. 31. If these are compared to the experimental curves in Fig. 8, some minor differences are seen. Probably due to the simplified material model for the projectile, giving a too small plastic deformation of the nose during impact, the slope of the calculated residual versus impact velocity curves is slightly steeper than in the corresponding experimental curves for the thickest targets. No fracture criterion is included in the material model for the projectile. Hence, the residual versus impact velocity curves for 20,25 and $30 \mathrm{~mm}$ thick target plates could be simulated without problems. The jump in residual velocity at the ballistic limit seems to decrease slightly with target thickness, as also observed experimentally, and the agreement between numerical and experimental curves is in general good. Both the residual versus impact velocity curves and ballistic limit velocities were estimated from a least square fit of the model constants in the RechtIpson model in Eq. (1) to the numerical residual velocities. Values of a, $\mathrm{p}$ and $v_{\mathrm{bl}}$ from the fits are given in Table 8. Ballistic limit velocities are plotted against target thickness and compared to 


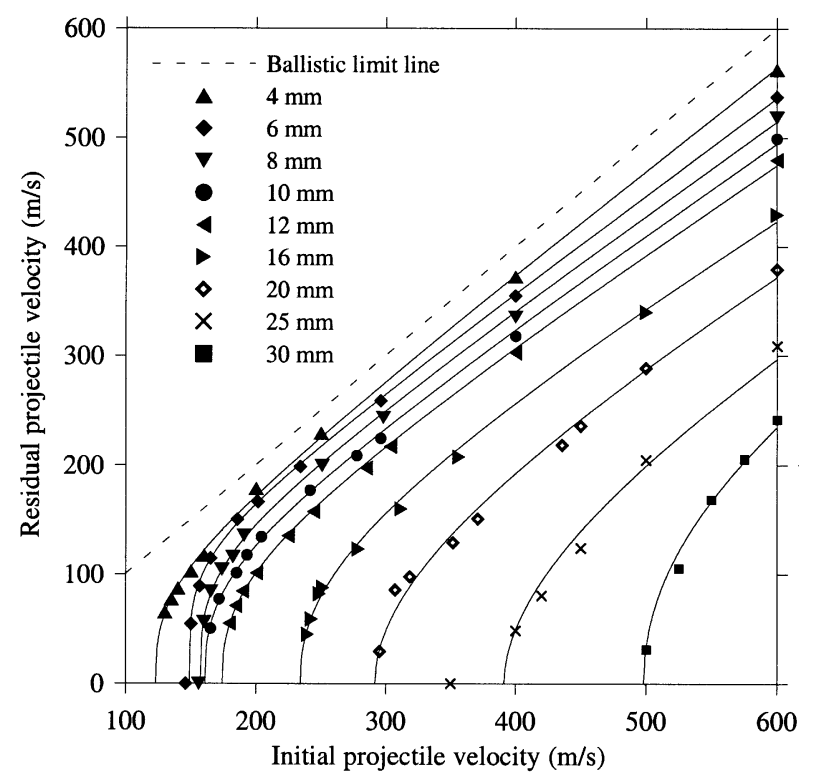

Fig. 31. Residual versus impact velocity curves obtained from numerical simulations.

Table 8

Numerically obtained ballistic limit velocities versus target thickness

\begin{tabular}{|c|c|c|c|c|c|c|c|c|c|}
\hline & \multicolumn{9}{|c|}{ Target thickness (mm) } \\
\hline & 4 & 6 & 8 & 10 & 12 & 16 & 20 & 25 & 30 \\
\hline$v_{\mathrm{bl}}(\mathrm{m} / \mathrm{s})$ & 123.3 & 149.1 & 157.6 & 161.1 & 174.3 & 234.4 & 291.9 & 391.4 & 498.5 \\
\hline $\mathrm{a}$ & 0.94 & 0.89 & 0.86 & 0.83 & 0.80 & 0.74 & 0.72 & 0.68 & 0.66 \\
\hline $\mathrm{p}$ & 2.91 & 4.09 & 3.40 & 2.81 & 2.59 & 2.31 & 1.95 & 1.86 & 2.13 \\
\hline
\end{tabular}

experimental values in Fig. 32. The agreement is excellent and the numerical predictions are within $5 \%$ of the corresponding experimental value for all target thicknesses. The simulations also give a precise description of the observed kink in response curve with target thickness.

Plots showing perforation of target plates with different thicknesses are given in Fig. 33, where fringes of accumulated plastic strain are plotted on the deformed geometry. It is seen that the extension of the localised plastic zone increases and the global target deformation decreases with increasing target thickness (see also [24]). These plots compare well with the high-speed camera images in Fig. 12, except for the 25 and $30 \mathrm{~mm}$ thick targets where the projectiles fractured at impact. Thus, the numerical approach seems to capture the overall physical behaviour of the targets during plugging. Details of the shear localisation, crack propagation and complete failure in the element mesh from one simulations of a $20 \mathrm{~mm}$ thick target are shown in Fig. 34. The number of eroded elements in simulations using blunt projectile is normally small. In general, as the initial projectile velocity is increased, so is the number of eroded elements. For the thinnest targets, where much tension is present during failure, the number of eroded elements is relatively higher than for thicker plates dominated by shear. As the target becomes thick, the extension of 


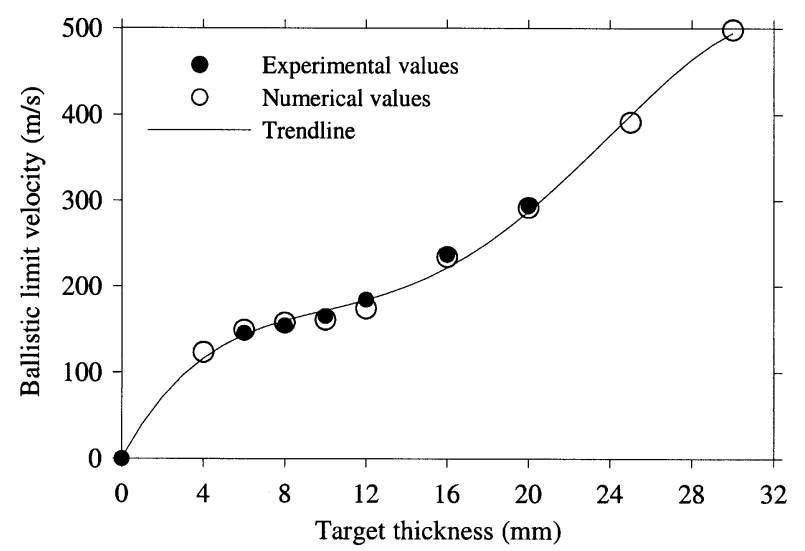

Fig. 32. Ballistic limit velocity versus target thickness from numerical simulations.

the plastic zone causes more than one element column to erode. This can be seen from the fracture patterns in Fig. 35 for different target thicknesses at impact velocities close to the ballistic limit. Even so, the loss of energy in the simulations is small and normally not more than $1-2 \%$ of the initial kinetic energy. The energy loss due to element erosion has been discussed in more detail by Børvik et al. [29].

Calculated histories of projectile velocity and interface force are plotted in Fig. 36. If the velocity-time curves are compared with those measured from the high-speed camera images in Fig. 16, the behaviour is found to be similar. Both figures indicate that the perforation time is rather constant and rather independent of target thickness when the impact velocity is close to the ballistic limit. The small areas of almost constant velocity seen for all target thicknesses in Fig. 36(a) are due to the temporary loss of contact between the projectile and plug during penetration. This is probably the reason for the observed difference in projectile and plug residual velocity [12]. The extensive drop in velocity over a short time period implies a large interface force at impact (Fig. 36(b)). This large interface force seems to be the main reason for the projectile break-up in the thickest targets. In thinner plates, on the other hand, the impulse is not strong enough to cause projectile fragmentation. Once the material in front of the projectile starts to move, the force falls off rapidly. From Fig. 36(b) it is seen that the force does not drop to zero immediately after fracture. The force continues to act as long as the plug and projectile nose are within the target due to contact between the bodies (as illustrated in Fig. 34).

Permanent plug thickness versus initial target thickness from the simulations is plotted in Fig. 37 and several interesting features are obtained. First, the computed thinning of the plug is almost linear and in close agreement with the experimental values to a target thickness of about $20 \mathrm{~mm}$ (see also Fig. 17(a)). Then, for thicker plates the plug thinning seems to flatten out, indicating a constant plug thickness. Børvik et al. [28,29] studied the effect of projectile nose shape on the failure mode in targets both experimentally and numerically. They found that in $12 \mathrm{~mm}$ thick Weldox 460 E steel plates, plugging due to adiabatic shear banding was the most effective failure mode. From a simplified model by Woodward [37], it can be shown analytically that plugging is more favourable than ductile hole enlargement for a target thickness less than $\sqrt{3} d_{\mathrm{p}} / 2$, which in this particular study is about $17 \mathrm{~mm}$. For targets with a thickness above $20 \mathrm{~mm}$, the 


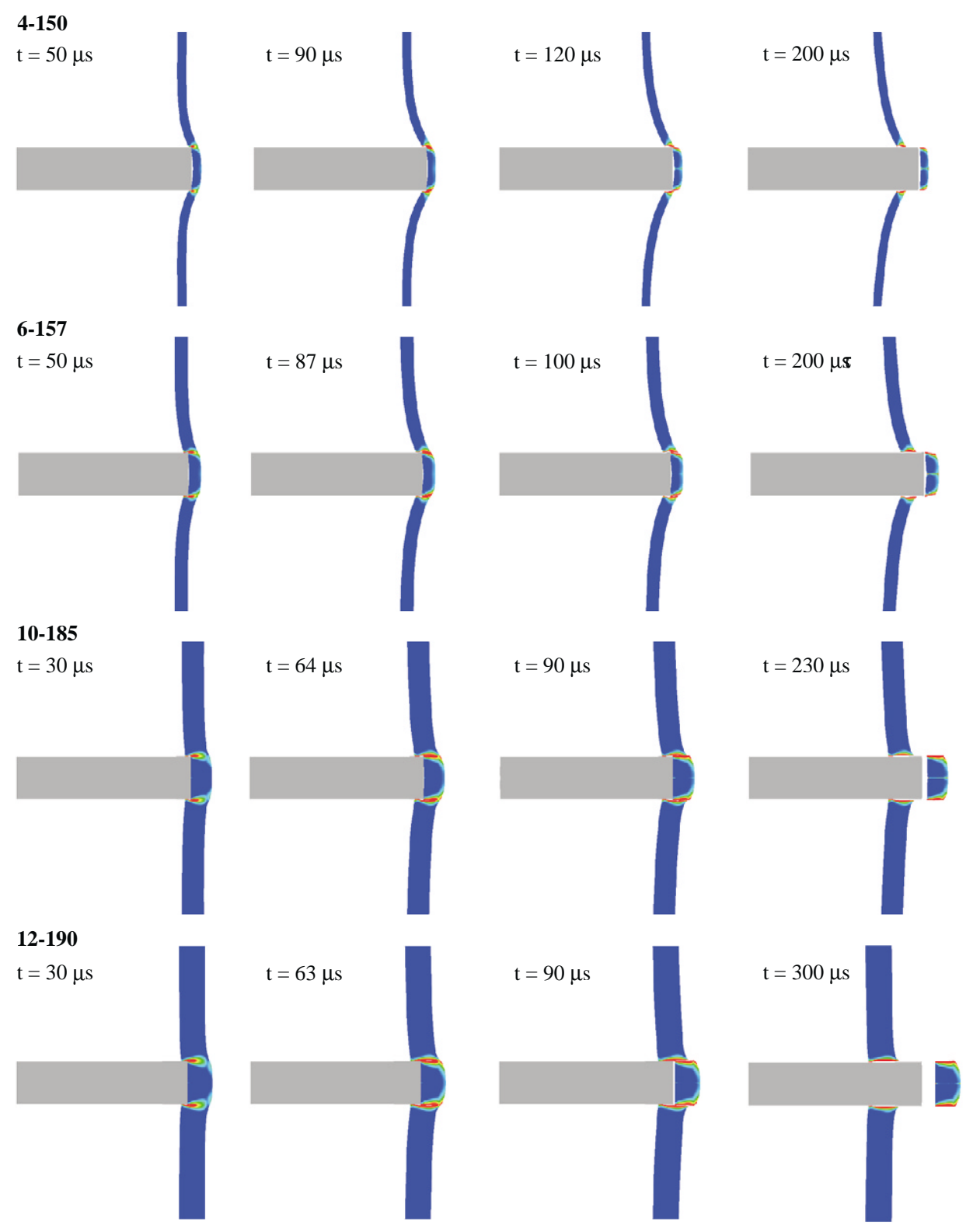

Fig. 33. Numerical simulations of plugging plotted as fringes of effective plastic strain.

material in front of the projectile is pushed aside during indentation due to ductile hole enlargement. Thus, when the thickness of the target in front of the projectile has been sufficiently reduced, plugging will again be the dominant failure mode and a plug failure occurs.

The amount of energy absorbed in local versus global target deformation and in projectile mushrooming in simulations at impact velocities close to the respective ballistic limits is shown in Fig. 38 for different target thicknesses. For the thinnest plates, most of the energy is absorbed in 


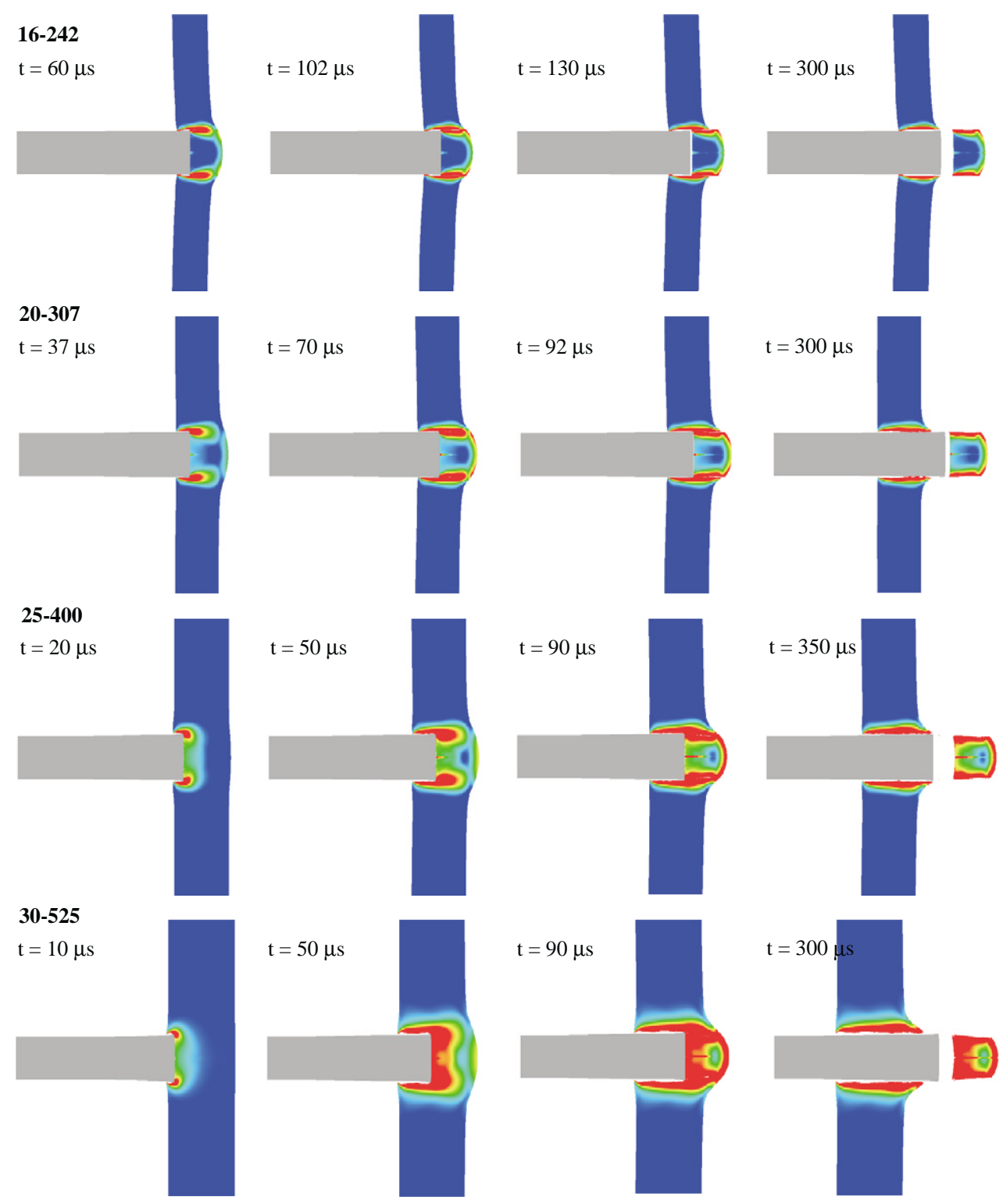

Fringe levels of effective plastic strain:

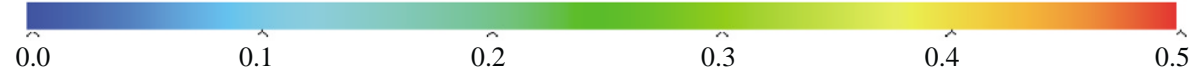

Fig. 33 (continued).

global deformation. However, as the thickness is increased, more and more energy is absorbed in localised shearing. At a target thickness of $10 \mathrm{~mm}$, the curves intersect and more energy is absorbed in local than in global deformation. This is also where the deformation mode was found 

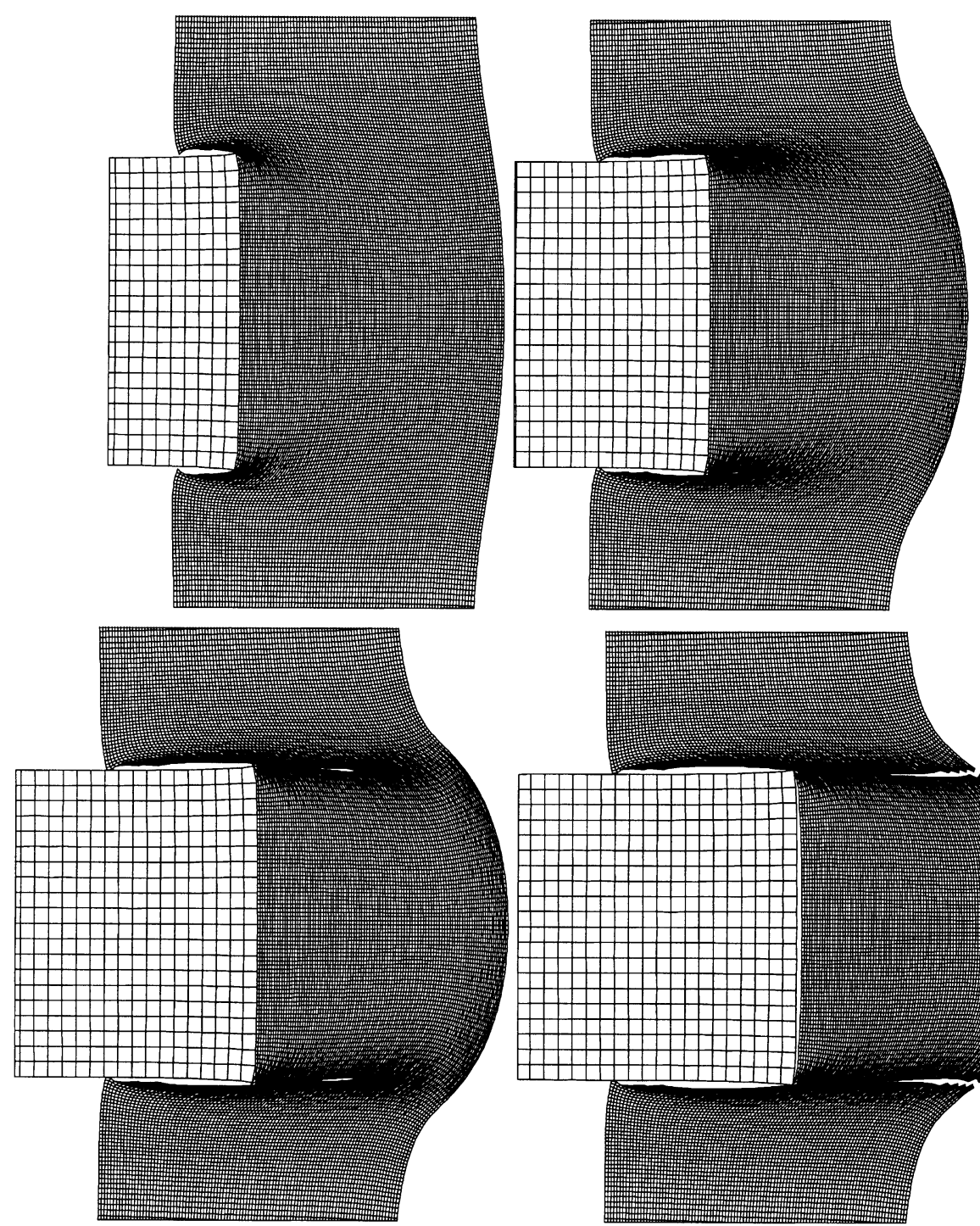

Fig. 34. Details of the crack propagation and failure in Run \# 20-307.

to change (see Fig. 9), and where localised adiabatic shear bands were first detected (see [24]). Up to a thickness of $16 \mathrm{~mm}$, the amount of local work continues to increase, while the amount of global work decreases. Afterwards, the ratio between the two curves is rather constant. The amount of energy absorbed by the projectile is found to increase linearly to a target thickness of about $20 \mathrm{~mm}$. This is where the projectile starts to fracture in the tests. The energy absorption then flattens out and becomes constant at about $20 \%$ of the total work. Liu and Stronge [49] did not find such a steady increase in projectile work with increasing plate thickness in their experimental tests using deformable aluminium projectiles to impact aluminium plates. They used a model proposed by Johnson [50] to calculate the amount of energy absorbed by the projectile, 
$30-525$

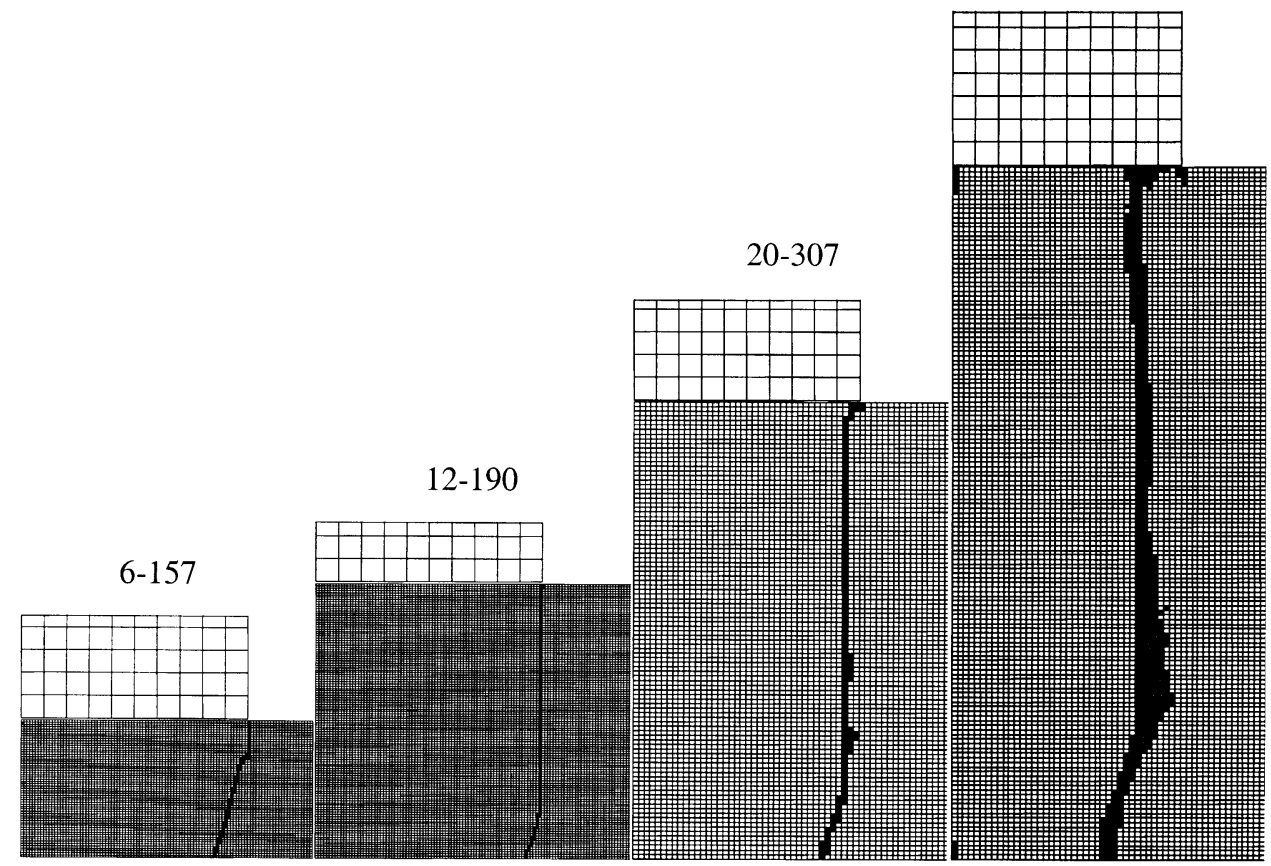

Fig. 35. Plots of removed elements (shown as black areas) forming the fracture patterns for different target thicknesses at impact velocities close to the respective ballistic limit velocity.

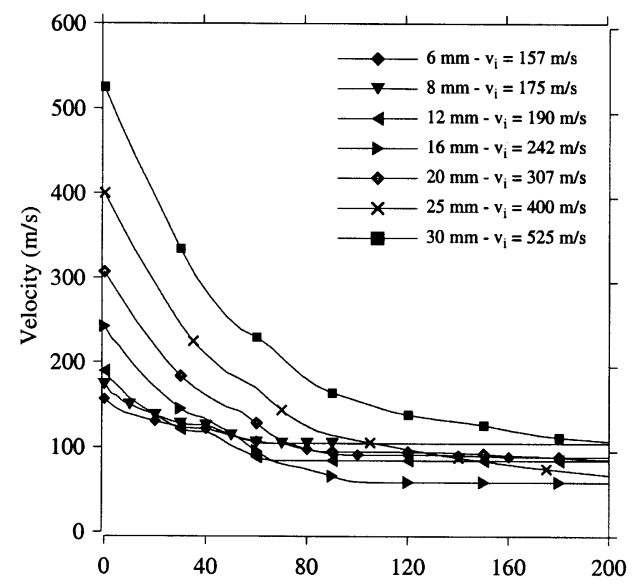

(a)

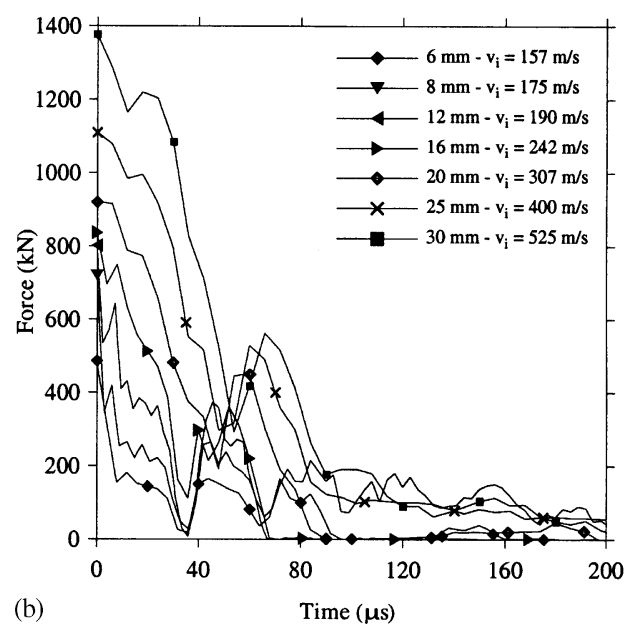

Fig. 36. Projectile (a) velocity and (b) interface force versus time for targets of different thickness during perforation from numerical simulations at impact velocities about $5 \%$ above the respective ballistic limit velocity.

assuming that the material is rigid perfectly plastic. The idea of this model is to calculate the plastic work necessary to deform a blunt-ended cylinder into an idealised frustum by making postimpact measurements of the deformed shape. Johnson's model [50] has also been used in this 


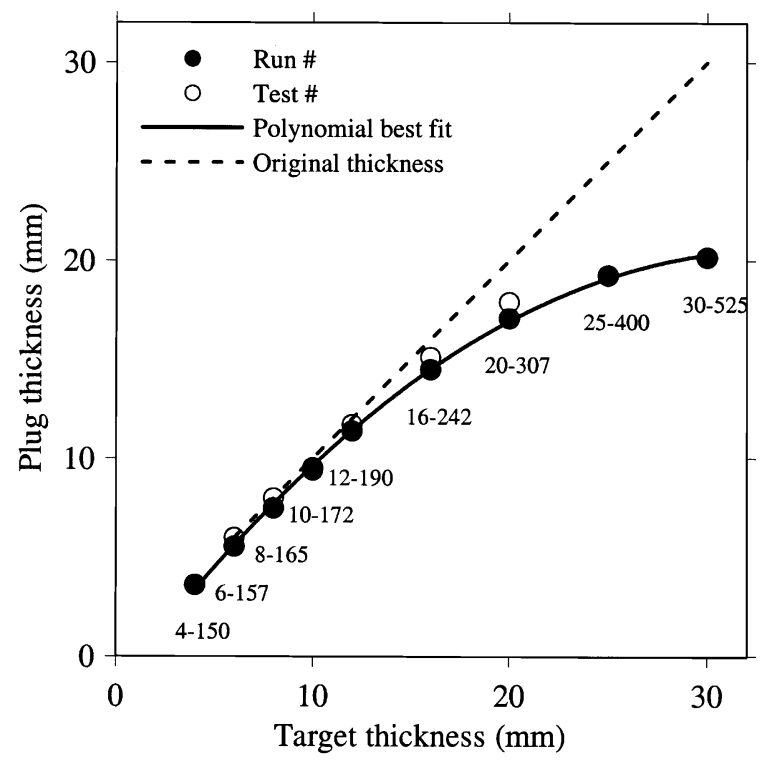

Fig. 37. Plug thickness versus target thickness from numerical simulations at impact velocities close to the respective ballistic limits (see also Figs. 17 and 33).

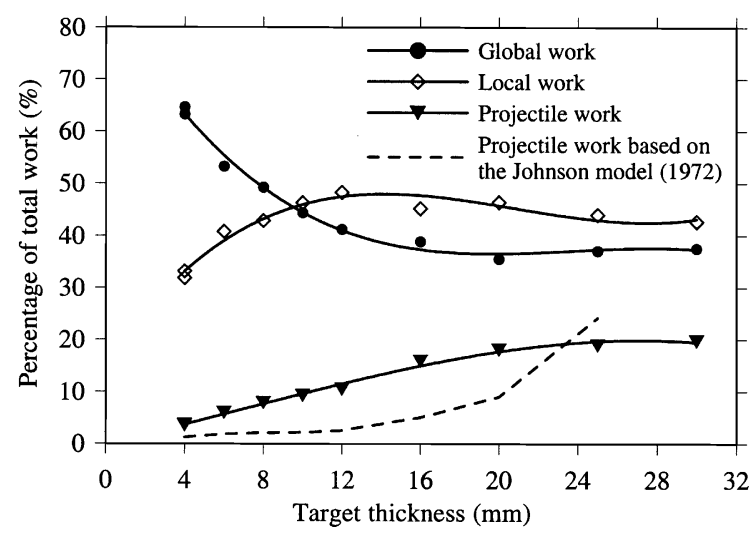

Fig. 38. Energy absorbed in local and global target deformation and in projectile mushrooming as a function of target thickness, from numerical simulations at impact velocities close to the respective ballistic limits.

study, but here it is assumed that the projectile material exhibits linear work hardening. Postimpact measurements of the deformed shape can be found in Table 2 (and Fig. 18), material properties for the projectile are given in Table 1 (and Fig. 5), while the model is presented in Appendix C. Calculations using this model is plotted and compared with the numerical results in Fig. 38. As seen, there is some disagreement in the results between the two methods, especially at the lowest impact velocities. Relative deformations versus target thickness from the numerical simulations have been plotted and compared to the experimental data and results from the WenJones analytical model in Fig. 10, and the agreement is good. Finally, perforation times versus 
target thicknesses are given in Fig. 27. Here, the experimental data points have been estimated from the high-speed camera images and are therefore only approximate. However, the numerical data indicates that the perforation time is reduced with increasing target thickness to $12 \mathrm{~mm}$, in contrast to calculations from the analytical models.

\section{Concluding remarks}

This paper is concerned with the ballistic penetration and perforation of circular Weldox $460 \mathrm{E}$ steel plates with thicknesses ranging from 6 to $30 \mathrm{~mm}$ impacted by blunt cylindrical projectiles in the velocity range of $75-500 \mathrm{~m} / \mathrm{s}$. The study is divided into three major parts. First, experimental details from almost 100 impact tests were given. In the tests, the only variables were the initial projectile velocity and the target thickness. In the second part, empirical equations and analytical models were briefly discussed, and then used to estimate ballistic limit velocities and residual versus impact velocity curves. The last part gave results and findings from more than 150 finite element simulations of the problem investigated experimentally. Some important observations and conclusions from the study are listed below.

- Experimentally, both the jump in residual velocity at the ballistic limit and the slope of the residual versus impact velocity curve seems to decrease with target thickness and initial projectile velocity.

- A kink in the ballistic response curve was obtained at a target thickness of about $10 \mathrm{~mm}$. The change in target response seems related to a change in deformation mode with target thickness, from thin plate global deformation towards thick plate shear localisation. This behaviour was also verified in a metallographical investigation [24].

- While the maximum target deformation was nearly twice the thickness for the thinnest plates, hardly any global deformation could be measured for plates of $16 \mathrm{~mm}$ or thicker. Projectile deformation, on the other hand, increased strongly with target thickness and impact velocity. At thicknesses above $20 \mathrm{~mm}$, the projectile fractured at impact with only limited damage on the target plate. The perforation time was found to be fairly constant for target thicknesses $h_{\mathrm{t}} \geqslant 8 \mathrm{~mm}$ at impact velocities close to the ballistic limit.

- Calculations using empirical equations revealed an extensive spread in model results and a considerable difference between predictions and experiments. It is realised that most empirical models result from curve fits to a limited number of experimental data and do not provide any physically based description. Even though the empirical approach is still frequently used for design purposes, mainly due to the absence of reliable analytical models for preliminary calculations, they should not be used without special care.

- Three typical analytical models were also used in calculations of the ballistic event. Unfortunately, none of these were able to describe the structural response correctly when the target thickness changed from thin to thick, even though all models gave results in reasonable agreement with the experimental data. The reason why analytical models sometimes fail to describe the structural response may be a poor description of the material behaviour under impact loading or the fact that many analytical models assume one physical mechanism to dominate the problem. Nevertheless, it was found that the Wen-Jones model gives a good 
prediction of the global target deformation and reasonable results for thin and intermediate thick plates. The Bai-Johnson model, on the other hand, gives a physical description of shear instability, and results in reasonable agreement with the experimental data for the thickest targets. Although the Ravid-Bodner model showed deviation compared to the experimental results, it is by far the most general of the analytical models used. It is expected to give better results when the impact velocity is increased, such as the case of armour piercing bullets impacting hard steel targets.

- A large number of numerical simulations have been carried out using LS-DYNA. When the numerical results are compared with the experimental data, excellent agreement is found. Both the residual versus impact velocity curves and in particular the ballistic limit velocities are precisely determined using numerical simulations. Also the overall physical behaviour of the target plate during impact is very well described.

- The amount of detailed information from the numerical simulations is overwhelming, and in general much more extensive than what is available from analytical models. In particular the ability of visualising the penetration process in combination with a large number of history variables is advantageous. From the numerical results in this and similar papers, it seems reasonable to state that a verified and validated numerical model can be used both in design and to increase the physical understanding of the penetration problem.

\section{Appendix A. The Wen-Jones [31] model}

Consider a clamped circular plate having an outer radius $R$ and thickness $H$ loaded at the centre by a blunt circular punch with diameter $d=2 a$. It is assumed that a transverse velocity (or displacement) field for a rigid, perfectly plastic plate can be written as

$$
W=W_{0} \text { for } r \leqslant a \text { and } W=W_{0}\left[\frac{\ln (r / R)}{\ln (a / R)}\right] \text { for } a \leqslant r \leqslant R,
$$

where $W$ is the transverse displacement of the plate at a radius $r$ and $W_{0}$ is the transverse displacement at the periphery of the punch-plate interface. Taking local indentation $\Delta$ into account and utilising the principle of virtual work, an approximate quasi-static load-displacement relationship can be established as

$$
W_{\mathrm{t}}=W_{0}+\Delta=\frac{F-F_{\mathrm{c}}}{K_{\mathrm{m}}}+\left(\frac{F}{F_{\mathrm{u}}}\right)^{1 / n} \Delta_{\mathrm{c}}
$$

where $W_{\mathrm{t}}$ is the total transverse displacement of the punch, $F$ is the applied load between the punch and plate, $F_{\mathrm{c}}=(4 / \sqrt{3}) \pi M_{0}[1+(1+\sqrt{3} / 2) / \ln (R / a)]$ is the plate's static collapse load for infinitesimal displacements and $K_{\mathrm{m}}=2 \pi N_{0} / \ln (R / a)$ is the membrane stiffness of the plate. $N_{0}=$ $\sigma_{\mathrm{y}} H$ and $M_{0}=\sigma_{\mathrm{y}} H^{2} / 4$ are the fully plastic membrane force and bending moment per unit length, respectively, and $\sigma_{\mathrm{y}}$ is the static uniaxial tensile yield stress. $F_{\mathrm{u}}=\tau_{\mathrm{u}} A_{\mathrm{s}}$ gives the critical shear force at plugging failure, where $\tau_{\mathrm{u}}=\sigma_{\mathrm{u}}[0.41(H / d)+0.42]$ may be taken as the critical shear stress for steel plates [7]. Finally, $n$ is the work-hardening index of the material defined from the constitutive expression $\tau=\tau_{\mathrm{u}}\left(\gamma / \gamma_{\mathrm{u}}\right)^{n}$. The relation between the punch indentation $\Delta$ and the shear strain $\gamma$ is 
assumed as

$$
\Delta=e \gamma=\frac{1+\sqrt{3}}{8} H \gamma
$$

where $e$ is the half-width of the localised shear band, obtained using moment equilibrium for an axisymmetrically loaded circular plate. Thus, the critical indentation $\Delta_{\mathrm{c}}$ of the punch when shear failure occurs is $\Delta_{\mathrm{c}}=e \gamma_{\mathrm{u}}$, in which $\gamma_{\mathrm{u}}$ is the critical shear strain. The energy is assumed absorbed by the system when statically loaded as

$$
E_{\mathrm{t}}=E_{\mathrm{bm}}+E_{\mathrm{s}}=\frac{F_{\mathrm{u}}^{2}-F_{\mathrm{c}}^{2}}{2 K_{\mathrm{m}}}+\frac{F_{\mathrm{u}} \Delta_{\mathrm{c}}}{1+n}
$$

or equivalent

$$
\frac{E_{\mathrm{t}}}{\sigma_{\mathrm{y}} d^{3}}=A\left(\frac{H}{d}\right)+B\left(\frac{H}{d}\right)^{2}+C\left(\frac{H}{d}\right)^{3}
$$

where

$$
\begin{aligned}
& A=0.138 \lambda^{2} \ln (R / a), \\
& B=0.270 \lambda^{2} \ln (R / a)+0.451 \lambda \gamma_{\mathrm{u}} /(1+n), \\
& C=0.132 \lambda^{2} \ln (R / a)+0.440 \lambda \gamma_{\mathrm{u}} /(1+n)-(\pi / 12) \ln (R / a)[1+(1+\sqrt{3 / 2}) / \ln (R / a)]^{2} .
\end{aligned}
$$

Here, $E_{\mathrm{bm}}$ is the energy dissipated by bending and membrane stretching, $E_{\mathrm{s}}$ is the energy dissipated by shear deformations, and $\lambda=\sigma_{\mathrm{u}} / \sigma_{\mathrm{y}}$ where $\sigma_{\mathrm{u}}$ is the ultimate tensile stress. It was further assumed that a quasi-static model was suitable for metal plates impacted by relatively large masses travelling at relatively low velocities. Material strain rate sensitivity was taken into account using the Cowper-Symonds equation

$$
\sigma_{\mathrm{d}}=\sigma_{\mathrm{y}}\left[1+\left(\frac{\dot{\varepsilon}_{\mathrm{m}}}{D}\right)^{1 / q}\right], \quad \dot{\varepsilon}_{\mathrm{m}}=\frac{2 W_{\mathrm{os}} V_{\mathrm{c}}}{3 \sqrt{2} \operatorname{Ra} \ln ^{2}(a / R)}, \quad W_{\mathrm{os}}=\frac{F_{\mathrm{u}}-F_{\mathrm{c}}}{K_{\mathrm{m}}}
$$

where $\sigma_{\mathrm{d}}$ is the dynamic yield stress, $D$ and $q$ are material constants, $\dot{\varepsilon}_{\mathrm{m}}$ is the mean strain rate when the plate fails in shear plugging, $V_{\mathrm{c}}$ is the critical velocity or ballistic limit, and $W_{\mathrm{os}}$ is the critical transverse displacement at failure. At the ballistic limit, the amount of energy absorbed by the system is $E_{\mathrm{t}}=E_{\mathrm{p}}=\frac{1}{2} G V_{\mathrm{c}}^{2}$, where $G$ is the projectile mass. Thus, an approximate expression of the ballistic limit for the target is found from Eq. (A.5) as

$$
V_{\mathrm{c}}=\sqrt{\frac{2 \sigma_{\mathrm{y}} d^{3}}{G}}\left(\left[1+\left(\frac{2 W_{\mathrm{os}} V_{\mathrm{c}}}{3 \sqrt{2} D R a \ln ^{2}(a / R)}\right)^{1 / q}\right]\left[A\left(\frac{H}{d}\right)+B\left(\frac{H}{d}\right)^{2}+C\left(\frac{H}{d}\right)^{3}\right]\right)^{1 / 2} .
$$




\section{Appendix B. The Bai-Johnson [36] model}

Consider the normal impact of an infinite circular plate with thickness $b$ and density $\rho$ by a blunt, cylindrical projectile with radius $a$ and mass $M$. The distance $r$ is zero in the centre of the plate and $r=a$ at the periphery of the projectile. It is assumed that only simple shear deformation occurs along the direction of the moving projectile during the penetration process, and friction and stress wave effects are neglected. The displacement of the plate element at $r=a$, which equals the penetration depth $p$ of the projectile, can be obtained by the integral

$$
p=-\int_{\infty}^{a} \gamma \mathrm{d} r
$$

This limits the validity of the model to systems where the ratio of projectile diameter to target thickness is about unity. The conservation laws give the relationship between the initial velocity of the projectile $v_{0}$ and the current velocity of the projectile and plug $v$ as

$$
v=\left\{\frac{M^{2}}{(M+m)^{2}} v_{0}^{2}-\frac{2 e}{(M+m)}\right\}^{1 / 2},
$$

where $m=\pi a^{2} b \rho$ is the mass of the plug. The energy $e$ absorbed in the shear zone as a function of penetration depth $p$ is calculated as

$$
e(p)=\int_{0}^{p} 2 \pi a b \tau_{a}(\bar{p}) \mathrm{d} \bar{p}
$$

where $\tau_{a}$ is the shear stress at distance $r=a$, which is at the plug boundary. The function $\tau_{a}=\tau_{a}(p)$ is derived below. An expression for the ballistic limit or critical impact velocity $v_{0 C}$, which implies zero residual velocity of the projectile, is readily obtained as

$$
v_{0 C}=\left\{2 e_{\mathrm{u}}(M+m) / M^{2}\right\}^{1 / 2},
$$

where $m=\pi a^{2} b \rho$ is the mass of the plug, $e_{\mathrm{u}}=e\left(p_{\mathrm{u}}\right)$ is the ultimate energy absorbed in the shear zone during the impact event, and $p_{\mathrm{u}}$ is the ultimate penetration beyond which no energy is consumed. Bai and Johnson used as a criterion for shear instability that temperature softening should outbalance strain and strain-rate hardening, i.e. that an increment in shear stress was equal to or less than zero in the loading process. They also assumed that the effect of strain rate variation under adiabatic conditions could be ignored. Using these assumptions, an explicit expression of the shear stress in terms of the shear strain was proposed as

$$
\tau=\tau_{\mathrm{M}}\left(\frac{\gamma}{\gamma_{\mathrm{u}}}\right)^{n} \exp \left\{\frac{n}{1+n}\left[1-\left(\frac{\gamma}{\gamma_{\mathrm{u}}}\right)^{n+1}\right]\right\},
$$

where $\gamma_{\mathrm{u}}$ and $\tau_{\mathrm{M}}$ denote the critical shear strain and the maximum shear stress at incipient thermoplastic shear instability, i.e. at $\mathrm{d} \tau=0$, and $n$ represents the strain hardening exponent. In the derivation of the constitutive relation, it was assumed that $K=1$, i.e. the portion of plastic work converted into heat equals unity. The differential equation of the target plate, assuming the deformation of the plate beyond the plug to be quasi-static, gives the relation between the shear stress $\tau$ and the distance $r$. Using the constitutive relation, an expression for the shear strain $\gamma$ as a function of distance $r$ is obtained. Since this expression turns out to be rather involved, Bai and 
Johnson proposed an approximation in the form

$$
\frac{\gamma}{\gamma_{a}}=\left(\frac{a}{r}\right)^{1 / n}
$$

The relation between the shear strain $\gamma_{a}$ at distance $r=a$ (i.e. in the adiabatic shear band) and the penetration depth $p$ follows as

$$
\gamma_{a}(p)=\frac{1-n}{n} \frac{p}{a}
$$

Time differentiation of Eq. (B.7) gives the shear strain rate in the adiabatic shear band

$$
\frac{\mathrm{d} \gamma_{a}}{\mathrm{~d} t}=\frac{1-n}{n} \frac{\mathrm{d} p_{a}}{a \mathrm{~d} t}=\frac{1-n}{n} \frac{v_{a}}{a}
$$

Combining the above equations, it is possible to express the shear stress $\tau_{a}$ in terms of the penetration depth $p$

$$
\tau_{a}(p)=\tau_{\mathrm{M}}\left(\frac{1-n}{n \gamma_{\mathrm{u}}} \frac{p}{a}\right)^{n} \exp \left\{\frac{n}{1+n}\left[1-\left(\frac{1-n}{n \gamma_{\mathrm{u}}} \frac{p}{a}\right)^{n+1}\right]\right\} .
$$

It is further possible to obtain an expression for the temperature $\theta_{a}$ at the plug boundary, $r=a$, as a function of the penetration depth $p$

$$
\theta_{a}(p)=\theta_{0}+\frac{1-n}{n a} \frac{1}{\rho C_{V}} \int_{0}^{p} \tau_{a}(\bar{p}) \mathrm{d} \bar{p},
$$

where $\theta_{0}$ is the initial temperature of the target and $K$ equals unity. The penetration time $t$ against penetration depth $p$ can finally be calculated from the relation $\mathrm{d} p / \mathrm{d} t=v$ as

$$
t(p)=\int_{0}^{p} \frac{1}{v(\bar{p})} \mathrm{d} \bar{p} .
$$

\section{Appendix C. The Johnson [50] model}

It is possible to estimate the amount of energy absorbed in the projectile by making post-impact measurements of the deformed shape [50]. The idea is to calculate the amount of energy necessary to deform a cylindrical blunt-ended projectile into the idealised frustum shape seen in Fig. 39. The height of the frustum is difficult to measure, but by making use of the incompressibility hypothesis of plasticity, i.e. that plastic flow takes place at constant volume, the frustum height can be estimated as (see Fig. 39 for notation)

$$
h_{\mathrm{f}}=\frac{3 D^{2}\left(L-L_{\mathrm{f}}\right)}{D_{\mathrm{f}}^{2}+D D_{\mathrm{f}}-2 D^{2}} .
$$

Considering the convention of a cylinder into a conical frustum, the plastic work for an element slice is given as

$$
\mathrm{d} W=\mathrm{d} V \int_{0}^{\varepsilon} \sigma \mathrm{d} \varepsilon
$$




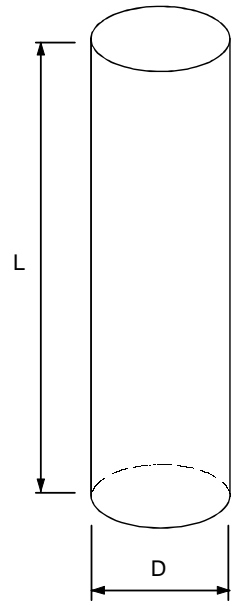

(a)

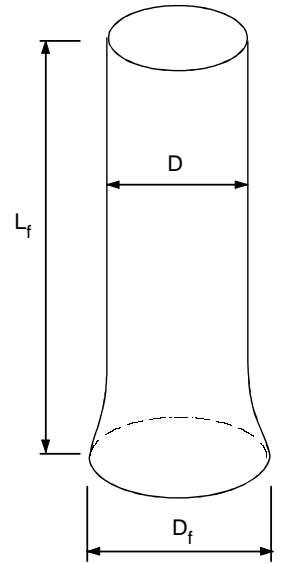

(b)

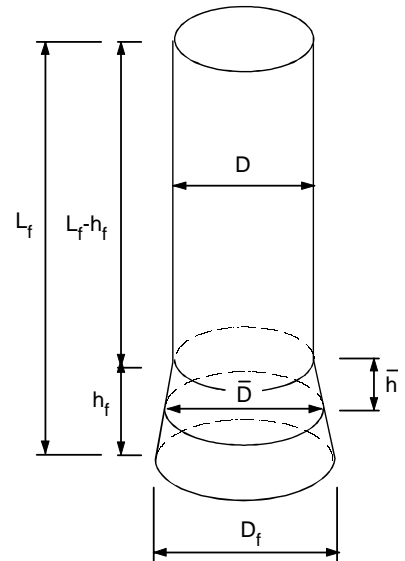

(c)

Fig. 39. Different projectile profiles (a) initial, (b) mushroomed, (c) idealised.

A constitutive equation for the projectile on the form

$$
\sigma=\sigma_{\mathrm{y}}+E_{\mathrm{t}} \varepsilon^{n}
$$

is known to give a good fit to many stress-strain curves. Here, the hardening is assumed linear (see Fig. 5) so the hardening parameter $n$ is set equal to 1 . The true strain is expressed as

$$
\varepsilon=2 \ln (\bar{D} / D)
$$

and one volume element is

$$
\mathrm{d} V=\frac{\pi \bar{D}^{2}}{4} \mathrm{~d} \bar{h}
$$

From geometrical considerations it is possible to write (Fig. 39)

$$
\frac{\bar{D}}{D}=\left[1+\left(\frac{D_{\mathrm{f}}}{D}-1\right) \frac{\bar{h}}{h_{\mathrm{f}}}\right]
$$

Insertion of these expressions in the work equation and integration leads to the following expression for the energy absorption in the projectile during impact [50]:

$$
W_{\mathrm{p}}=\frac{\pi D_{\mathrm{f}}^{3} h_{\mathrm{f}}}{18\left(D_{\mathrm{f}}-D\right)}\left[\sigma_{\mathrm{y}}\left(3 \ln \frac{D_{\mathrm{f}}}{D}-\frac{D_{\mathrm{f}}^{3}-D^{3}}{D_{\mathrm{f}}^{3}}\right)+E_{\mathrm{t}}\left(\left\{3 \ln \frac{D_{\mathrm{f}}}{D}-2\right\} \ln \frac{D_{\mathrm{f}}}{D}+\frac{2}{3}\left(\frac{D_{\mathrm{f}}^{3}-D^{3}}{D_{\mathrm{f}}^{3}}\right)\right)\right] .
$$

Based on Eq. (C.7), the measured initial and final projectile geometry (see Table 2) and the measured material properties of the projectile (see Fig. 5 and Table 1), the amount of energy absorbed by the projectile during impact can be estimated.

\section{References}

[1] Goldsmith W. Non-ideal projectile impact on targets. Int J Impact Eng 1999;22(2-3):95-395.

[2] Backman ME, Goldsmith W. The mechanics of penetration of projectiles into targets. Int J Eng Sci 1987;16:1-99. 
[3] Corbett GG, Reid SR, Johnson W. Impact loading of plates and shells by free-flying projectile. Int J Impact Eng 1996;18(2):141-230.

[4] Zukas JA, et al. Impact dynamics. New York: Wiley, 1982.

[5] Zukas JA, et al. High velocity impact dynamics. New York: Wiley, 1990.

[6] Wen H-M, Jones N. Experimental investigation into the dynamic plastic response and perforation of a clamped circular plate struck transversely by a mass. Proceedings of the Institution of Mechanical Engineers. J Mech Eng Sci 1994;208(C2):113-37.

[7] Langseth M, Larsen PK. Dropped objects' plugging capacity of steel plates: an experimental investigation. Int J Impact Eng 1990;9(3):289-316.

[8] Langseth M, Larsen PK. The behaviour of square steel plates subjected to a circular blunt ended load. Int J Impact Eng 1992;12(4):617-38.

[9] Langseth M, Larsen PK. Dropped objects' plugging capacity of aluminium alloy plates. Int J Impact Eng 1994;15(3):225-41.

[10] Langseth M, Hopperstad OS, Berstad T. Impact loading of plates. Validation of numerical simulations by testing. Int J Offshore Polar Eng 1999;9(1):10-5.

[11] Andersion Jr CE, editor. Hypervelocity impact. Proceedings of the 1998 Symposium: Huntsville, Alabama, November 17-19, 1998. Int J Impact Eng 1999;23(1):1-1024.

[12] Børvik T, Langseth M, Hopperstad OS, Malo KA. Ballistic penetration of steel plates. Int J Impact Eng 1999;22(9-10):855-87.

[13] Scheffler DR, Zukas JA. Practical aspects of numerical simulations of dynamic events: material interfaces. Int $\mathbf{J}$ Impact Eng 2000;24(8):821-42.

[14] Zukas JA, Scheffler DR. Practical aspects of numerical simulations of dynamic events: effect of meshing. Int $\mathbf{J}$ Impact Eng 2000;24(9):925-45.

[15] Børvik T, Holen K, Langseth M, Malo KA. An experimental set-up used in ballistic penetration. Proceedings of the Fifth International Symposium on Structures under Shock and Impact, Thessaloniki, Greece, 24-26 June, 1998a, pp. 683-92.

[16] Børvik T, Vestli K, Langseth M. Determination of projectile path during ballistic penetration by use of a highspeed digital camera. Proceedings of the Association for High-Speed Photography and Photonics Conference, Cambridge, UK, April, 1997.

[17] Førre B. Private communication. Department of Physics, Norwegian University of Science and Technology (NTNU), 2000. E-mail: bernt.forre $@$ phys.ntnu.no

[18] LSTC. LS-DYNA Theoretical Manual, 1998.

[19] LSTC. LS-DYNA User's Manual, Version 950, 1999.

[20] Børvik T, Hopperstad OS, Berstad T, Langseth M. A computational model of viscoplasticity and ductile damage for impact and penetration. Eur J Mech-A/Solids 2001;20:685-712.

[21] Børvik T, Langseth M. Quasi-static penetration of steel plates. Proceedings from Integrity, Reliability, Failure: An International Conference, University of Porto, Portugal, 19-22 July, $1999 \mathrm{~b}$.

[22] Corran RSJ, Shadbolt PJ, Ruiz C. Impact loading of plates - an experimental investigation. Int J Impact Eng 1983;1(1):3-22.

[23] Hanchak SJ, Forrestal MJ. Private communication, 1997.

[24] Børvik T, Leinum JR, Solberg JK, Hopperstad OS, Langseth M. Observations on shear plug formation in Weldox 460 E steel plates impacted by blunt-nosed projectiles. Int J Impact Eng 2001;25(6):553-72.

[25] Recht RF, Ipson TW. Ballistic perforation dynamics. J Appl Mech 1963;30:384-90.

[26] Forrestal MJ, Hanchak SJ. Perforation on HY-100 steel plates with $4340 R_{\mathrm{c}} 38$ and maraging T-250 steel rod projectiles. Int J Impact Eng 1999;22(9-10):923-33.

[27] Børvik T, Hopperstad OS, Berstad T, Langseth M. Numerical simulation of plugging failure in ballistic penetration. Int J Solids Struct 2001;38(34-35):6241-64.

[28] Børvik T, Langseth M, Hopperstad OS, Malo KA. Perforation of $12 \mathrm{~mm}$ thick steel plates by $20 \mathrm{~mm}$ diameter projectiles with blunt, hemispherical and conical noses, Part I: experimental study. Int J Impact Eng 2002;27(1): 19-35. 
[29] Børvik T, Hopperstad OS, Berstad T, Langseth M. Perforation of $12 \mathrm{~mm}$ thick steel plates by $20 \mathrm{~mm}$ diameter projectiles with blunt, hemispherical and conical noses, Part II: numerical simulations. Int J Impact Eng 2002;27(1):37-64.

[30] Børvik T, Langseth M, Hopperstad OS, Malo KA. Empirical equations for ballistic penetration of metal plates. Fortifikatorisk Notat No. 260/98, The Norwegian Defence Construction Service, Central Staff-Technical Division, Oslo, Norway, 1998b.

[31] Wen H-M, Jones N. Low-velocity perforation of punch-impact-loaded metal plates. J Pressure Vessel Technol 1996;118(2):181-7.

[32] Wen H-M, Jones N. In: Bulson PS, editor. Semi-empirical equations for the perforation of plates struck by a mass, Structures Under Shock and Impact (SUSI) II, Computational Mechanics Publications, 1992, pp. 369-80.

[33] Liu D, Stronge WJ. Perforation of rigid-plastic plate by blunt missile. Int J Impact Eng 1995;16(5-6):739-58.

[34] Jones N. Structural impact. Cambridge: Cambridge University Press, 1989.

[35] Lindholm US, Johnson GR. Strain-rate effects in metals at large shear strains. In: Mescall J, Weiss V, editors. Proceedings of the 29th Sagamore Army Material Research Conference "Material behaviour under high stress and ultrahigh loading rates", 19-23 July, New York, 1982. p. 61-79.

[36] Bai YL, Johnson W. Plugging: physical understanding and energy absorption. Metals Technol 1982;9:182-90.

[37] Woodward RL. The interrelation of failure modes observed in the penetration of metallic targets. Int J Impact Eng 1984;2(2):121-9.

[38] Ravid M, Bodner SR. Dynamic perforation of viscoplastic plates by rigid projectiles. Int J Impact Eng 1983;21(6):577-91.

[39] Bodner SR, Ravid M. Review and some applications of 2D analytical models of ballistic penetration. Proceedings Transient loading and response of structures, International Symposium honouring Mr. Arnfinn Jenssen, Trondheim, Norway, 25-27 May.

[40] Ravid M, Bodner SR, Holcman I. Penetration into thick targets - refinement of a 2D dynamic plasticity approach. Int J Impact Eng 1994;15(5):587-603.

[41] Rimat-Advanced Technologies Ltd. SHAY 1S - dynamic perforation of viscoplastic plates by rigid projectiles. Version 3.2. Israel: Hod Hashron, 1992.

[42] Dieter GE. Mechanical metallurgy. UK: McGraw-Hill Book Company, 1988.

[43] Johnson GR, Cook WH. A constitutive model and data for metals subjected to large strains, high strain rates and high temperatures. Proceedings of the Seventh International Symposium on Ballistics, The Hague, 1983.

[44] Johnson GR, Cook WH. Fracture characteristics of three metals subjected to various strains, strain rates, temperatures, and pressures. Int J Eng Fract Mech 1985;21:31-48.

[45] Camacho GT, Ortiz M. Adaptive Lagrangian modelling of ballistic penetration of metallic targets. Int J Comp Meth Appl Mech Eng 1997;142:269-301.

[46] Lemaitre J, Chaboche J-L. Mechanics of solid materials. Cambridge: Cambridge University Press, 1990.

[47] Lemaitre J. A short course in damage mechanics. Berlin: Springer, 1992.

[48] Bai YL, Dodd B. Adiabatic shear localization. Occurrence, theories and applications. Oxford: Pergamon Press, 1992.

[49] Liu D, Stronge WJ. Ballistic limit of metal plates struck by blunt deformable missiles: experiments. Int J Solids Struct 2000;37:1403-23.

[50] Johnson W. Impact strength of materials. London: Edward Arnold, Ltd, 1972. 